\title{
THE ASYMPTOTIC GROWTH OF TORSION HOMOLOGY FOR ARITHMETIC GROUPS
}

\author{
NICOLAS BERGERON ${ }^{1}$ AND AKSHAY VENKATESH ${ }^{2}$ \\ ${ }^{1}$ Institut de Mathématiques de Jussieu, Unité Mixte de Recherche 7586 du CNRS, \\ Université Pierre et Marie Curie, 4, place Jussieu 75252 Paris Cedex 05, France \\ (bergeron@math.jussieu.fr) URL: http://people.math.jussieu.fr/ bergeron \\ ${ }^{2}$ Department of Mathematics, Stanford University, Stanford, CA 94304, USA \\ (akshay@math.stanford.edu)
}

(Received 6 July 2010; revised 26 October 2011; accepted 26 October 2011; first published online 7 June 2012)

Abstract When does the amount of torsion in the homology of an arithmetic group grow exponentially with the covolume? We give many examples where this is the case, and conjecture precise conditions.

\section{Asymptotic torsion}

Let $\Gamma$ be a lattice in a semisimple Lie group $G$. The asymptotic behaviour of the Betti numbers $\operatorname{dim} H_{j}(\Gamma, \mathbb{C})$, when $\Gamma$ varies, has been studied by a number of authors. In particular, if $\Gamma_{N} \leqslant \Gamma$ is a decreasing sequence of subgroups, with trivial intersection, the quotient

$$
\frac{\operatorname{dim} H_{j}\left(\Gamma_{N}, \mathbb{C}\right)}{\left[\Gamma: \Gamma_{N}\right]}
$$

is known [48] to converge to the $j$ th $L^{2}$-Betti number of $\Gamma$; in particular, this is nonzero only when the rank $^{3}$ of $G$ equals the rank of a maximal compact subgroup $K \subset G$. For example, this is the case when $G=\mathrm{SL}_{2}(\mathbb{R})$, and $\Gamma$ is a Fuchsian group.

The purpose of this paper is to investigate the corresponding question when Betti numbers are replaced by the logarithm of the size of the torsion components of homology groups. The motivation comes from number theory; see $\S 1.6$.

\section{1.}

Locally symmetric spaces associated with $\mathrm{SL}_{2}(\mathbb{C})$ are hyperbolic 3-manifolds. Numerical experiments ([27], and unpublished data computed by Calegari and Dunfield in connection with [15]) as well as the work of Calegari and Mazur [19] suggest that arithmetic hyperbolic 3-manifolds should have a lot of torsion in their homology. Here are two such results:

${ }^{3}$ Here the rank is the complex rank, i.e. the maximal dimension of a Cartan algebra, split or otherwise. 
(1) (Taylor, [67, Theorem 4.2]). Let $n_{1}$ and $n_{2}$ be two nonnegative integers. Consider $S_{n_{1}, n_{2}}=\operatorname{Sym}^{n_{1}}\left(\mathbb{Z}[i]^{2}\right) \otimes_{\mathbb{Z}} \overline{\operatorname{Sym}^{n_{2}}\left(\mathbb{Z}[i]^{2}\right)}$ as an $\operatorname{SL}_{2}(\mathbb{Z}[i])$-module. If $n_{1} \neq n_{2}$, there exists a finite index (congruence) subgroup $\Gamma \subset \mathrm{SL}_{2}(\mathbb{Z}[i])$ such that the homology groups

$$
H_{1}\left(\Gamma ; S_{n_{1}, n_{2}}\right)
$$

have a nontrivial torsion subgroup.

(2) (Follows from Silver and Williams [65].) Let $k \subset \mathbb{S}^{3}$ be a hyperbolic knot whose Alexander polynomial has nontrivial Mahler measure (e.g. the figure eight knot). Denote by $M_{N}$ the $N$ th cyclic cover of $\mathbb{S}^{3}$ branched over $k$. We decompose $H_{1}\left(M_{N} ; \mathbb{Z}\right)$ as the direct sum of a free abelian group and a torsion subgroup $H_{1}\left(M_{N}\right)_{\text {tors }}$.

For sufficiently large $N$, the 3 -manifold $M_{N}$ is hyperbolic; moreover,

$$
\lim _{N \rightarrow+\infty} \frac{\log \left|H_{1}\left(M_{N}\right)_{\text {tors }}\right|}{N}>0 \text {. }
$$

\section{2 .}

It is convenient to define, for a semisimple Lie group $G$, the 'deficiency' $\delta(G)$ to be the difference $\operatorname{rank}(G)-\operatorname{rank}(K)$. The quantity $\delta$ is sometimes called the fundamental rank of $G$. It equals zero if and only if $G$ has discrete series, or, equivalently, if and only if $G$ has a compact Cartan subgroup. If $S$ is the global symmetric space $G / K$, we sometimes write $\delta(S)$ for $\delta(G)$.

Examples. $\delta=0$ for the groups $\mathrm{SL}_{2}(\mathbb{R}), \mathrm{SU}_{n, m}, \mathrm{SO}_{n, m}$ ( $n m$ even); $\delta=1$ for the groups $\mathrm{SL}_{2}(\mathbb{C}), \mathrm{SL}_{3}(\mathbb{R}), \mathrm{SO}_{n, m} n m$ odd (this is a complete list of almost simple groups with $\delta=1$, up to isogeny); $\delta=2$ for the groups $\mathrm{SL}_{5}(\mathbb{R}), E_{6}^{\text {split }}$.

Now assume that $\mathbf{G}$ is $\mathbb{Q}$-semisimple and $\Gamma \subset \mathbf{G}(\mathbb{Q})$ is a congruence lattice. Put $G=\mathbf{G}(\mathbb{R})$. We fix an 'arithmetic' $\Gamma$-module $M$; by this we mean that $M$ is a finite rank free $\mathbb{Z}$-module, and there exists an algebraic representation of $\mathbf{G}$ on $M \otimes \mathbb{Q}$ such that $\Gamma$ preserves $M$. We assume that $\mathbf{G}$ is anisotropic over $\mathbb{Q}$ (or equivalently, $\Gamma$ is cocompact in $G$ ), and consider a decreasing sequence of congruence subgroups $\Gamma_{N} \subset \Gamma_{N-1} \subset \cdots \subset \Gamma$ with the property that $\bigcap_{N} \Gamma_{N}=\{1\}$.

Conjecture 1.3. The limit

$$
\lim _{N} \frac{\log \left|H_{j}\left(\Gamma_{N}, M\right)_{\text {tors }}\right|}{\left[\Gamma: \Gamma_{N}\right]}
$$

exists for each $j$ and is zero unless $\delta(S)=1$ and $j=\frac{\operatorname{dim}(S)-1}{2}$. In that case, it is always positive and equal to a positive constant $c_{G, M}$ (explicitly described in §5.9) times the volume of $\Gamma \backslash S$.

This conjecture - which remains somewhat speculative at this stage - can be considered as predicting three different types of behaviour:

(1) If $\delta=0$, then there is little torsion, whereas $H_{j}\left(\Gamma_{N}, M \otimes \mathbb{Q}\right)$ is large; the torsion is almost entirely 'absorbed' by the characteristic 0 homology. 
(2) If $\delta=1$, then there is 'a lot' of torsion but $H_{j}\left(\Gamma_{N}, M \otimes \mathbb{Q}\right)$ is small.

(3) If $\delta \geqslant 2$, there is 'relatively little' ${ }^{4}$ torsion or characteristic 0 homology.

As for the degree $\frac{\operatorname{dim}(S)-1}{2}$, this is the 'middle dimension' for torsion classes: there is a duality between dimensions $j$ and $\operatorname{dim}(S)-1-j$. Compare the situation for the $L^{2}$-Betti numbers of $\Gamma$ : they vanish unless $\delta=0$, and even then are nonvanishing only in degree $\frac{\operatorname{dim}(S)}{2}$ (again the 'middle dimension').

We find it very likely that the restriction to congruence $\Gamma_{N}$ is essential. That the intersection of the $\Gamma_{N}$ is trivial is also likely essential. See $\S 8.3$ for some counterexamples in this direction.

In the present paper, we shall show a result in support of the 'large torsion' direction. With the notation as previously, we say that $M$ is strongly acyclic if the spectra of the (form) Laplacian on $M \otimes \mathbb{C}$-valued $i$-forms on $\Gamma_{N} \backslash S$ are uniformly bounded away from 0 , for all $N, i$. Notice that this implies that $H_{j}\left(\Gamma_{N}, M\right)$ is torsion.

Theorem 1.4. With the notation as previously, suppose that $\delta(S)=1$. Then strongly acyclic arithmetic $\Gamma$-modules $M$ always exist; moreover, for any such,

$$
\liminf _{N} \sum_{j} \frac{\log \left|H_{j}\left(\Gamma_{N}, M\right)_{\text {tors }}\right|}{\left[\Gamma: \Gamma_{N}\right]} \geqslant c_{G, M} \operatorname{vol}(\Gamma \backslash S)>0 .
$$

Here the sum is taken over integers $j$ with the same parity as $\frac{\operatorname{dim} S-1}{2}$.

Remark. This theorem is deduced from Theorem 4.5, which remains valid for any sequence of cocompact lattices such that the injectivity radius of $\Gamma_{N} \backslash S$ goes to infinity - i.e., $\Gamma_{N}$ need not be subgroups of a fixed lattice $\Gamma$. It is possible to formulate a more general version of Theorem 1.4 in that setting; however, this requires 'compatible' specifications of modules for each $\Gamma_{N}$.

Examples. The $\mathrm{SL}_{2}(\mathbb{Z}[i])$-module $S_{n_{1}, n_{2}}\left(n_{1}, n_{2} \in \mathbb{N}\right)$ considered in the first example of $\S 1.1$ is strongly acyclic precisely when $n_{1} \neq n_{2}$. (Our theorem does not apply to this case, since the lattice is not cocompact; a twisted variant where it applies is detailed in $\S 8.2$.) It is likely that using some of the ideas of [20], one could obtain corresponding results for certain sequences of subgroups of $\mathrm{SL}_{2}(\mathbb{Z}[i])$, but we do not attempt to do so.

In Theorem 1.4, we cannot in general isolate the degree which produces torsion except in certain low degree examples.

If $G=\mathrm{SL}_{3}(\mathbb{R})$ then $\lim \inf _{N} \frac{\log \left|H_{2}\left(\Gamma_{N}, M\right)_{\mathrm{tors}}\right|}{\left[\Gamma: \Gamma_{N}\right]}>0$.

If $G=\mathrm{SL}_{2}(\mathbb{C})$ then $\lim _{N} \frac{\log \left|H_{1}\left(\Gamma_{N}, M\right)_{\text {tors }}\right|}{\left[\Gamma: \Gamma_{N}\right]}=c_{G, M} \operatorname{vol}(\Gamma \backslash S)$.

Indeed, these refinements result from the following two observations:

- The proof of the theorem establishes more than (1.4.1); it will in fact show that

$$
\lim _{N} \sum_{j}(-1)^{j+\frac{\operatorname{dim}(S)-1}{2}} \frac{\log \left|H_{j}\left(\Gamma_{N}, M\right)_{\text {tors }}\right|}{\left[\Gamma: \Gamma_{N}\right]}=c_{G, M \operatorname{vol}(\Gamma \backslash S) .}
$$

\footnotetext{
${ }^{4}$ We mean this in the weakest possible sense: there is not exponential growth of torsion. This is not to suggest that there is no torsion, or that the torsion that exists is uninteresting; quite the contrary!
} 
- One can bound the torsion in $H_{0}(M)$ by a polynomial in the index $\left[\Gamma: \Gamma_{N}\right]$; see $\S 8.6$.

- One can bound the torsion in $H_{\operatorname{dim}(S)-1}(M)$, via Poincaré duality and the long exact sequence in homology associated with

$$
M \rightarrow M \rightarrow M / p M
$$

If one assumes the truth of the congruence subgroup property (CSP) for a cocompact lattice $\Gamma \leqslant \mathrm{SL}_{3}(\mathbb{R})$, we obtain in that case also $\lim _{N} \frac{\log \left|H_{2}\left(\Gamma_{N}, M\right)_{\text {tors }}\right|}{\left[\Gamma: \Gamma_{N}\right]}=c_{G, M} \operatorname{vol}(\Gamma \backslash S)$; in fact, the CSP and the homology exact sequence for (1.4.3) would imply that $H_{1}(\Gamma, M)$ is small.

\section{5 .}

The main tool used in the proof of Theorem 1.4 is not due to us. It is a remarkable result [55] of Müller; see also Bismut and Zhang [8] - a generalization of the 'Cheeger-Müller theorem' $[21,54]$. This result states, loosely speaking, that the size of torsion groups can be computed by analytic methods. Beyond this result, the other techniques are also not original and have been used in other contexts (see [46] for instance). These methods have a combinatorial counterpart, which we briefly discuss in $\S 7$; this generalizes the work of Silver and Williams, mentioned above.

We remark that by combinatorial methods it is in principle possible to verify the conclusion of Theorem 1.4 for any specific $\Gamma, M$ by direct computation, without appealing to Müller's theorem (cf. $\S 7.8$, Remark). However, such an analysis would be very complicated, and we have not attempted to carry it out in any case.

Recently, Müller has announced a result of similar nature to Theorem 1.4 but in a different aspect. He studies the homological torsion for a fixed cocompact lattice in $\mathrm{SL}_{2}(\mathbb{C})$ as the module $M$ varies. The paper has now appeared in print [53]; see also the companion paper [51] joint with Marshall.

Also, Sengun has carried out [63] extensive computations of the integral cohomology of Bianchi groups. His results appear to lend support to our conjectures.

Finally, Thang Le has stated a conjecture similar to ours, but for a smaller class of manifolds, and has announced a result implying the other direction of the inequality; the slides of the talks that he has given on that matter are available on line.

\section{6 .}

Our motivation for studying these questions is arithmetic.

In $\S 6$ we recall conjectures of Ash [2,3] and others [27,30,67] that torsion in the homology of arithmetic groups has arithmetic significance: very roughly, a mod $p$ torsion class in the homology of an arithmetic group parametrizes a field extension $K / \mathbb{Q}$ whose Galois group is a simple group of Lie type over $\mathbb{F}_{p}$. The quantity $\delta$ specifies the isomorphism class of $K \otimes \mathbb{R}$.

We make an attempt in $\S 6.5 .1$ to verify whether Conjecture 1.3 and Theorem 1.4 are compatible with Ash's conjectures: are there indeed more such field extensions $K / \mathbb{Q}$ when $\delta=1$ ? Our analysis is based on heuristics proposed by Bhargava [7] and is therefore speculative; nonetheless, it seems to us worthy of inclusion. To be precise, we show that Ash's conjectures and Bhargava's heuristics imply that the 'likelihood' of 
the existence of a mod $p$ class in the homology of $\Gamma \leqslant G$ should be of size $\sim p^{-\delta(G)}$. If $\delta(G)=0$, this amounts simply to the fact that there is abundant characteristic 0 homology, which reduces to giving abundant $\bmod p$ homology. On the other hand, since

$$
\sum_{p} p^{-m}= \begin{cases}\infty, & \text { if } m=1 \\ \text { finite, } & \text { if } m>1,\end{cases}
$$

this suggests an abundance of torsion precisely when $\delta(G)=1$.

\subsection{Organization of the paper}

In the body of the paper, we deal with cohomology rather than homology, since it is easier to make the transition between cohomology and differential forms. ${ }^{5}$

Section 2 is expository: a review of analytic and Reidemeister torsion. We discuss in detail the trivial example of cyclic covers of a circle (equipped with a nontrivial local system); although they are simple, many of the ideas that we use can be already seen clearly here.

Sections 3 and 4 prove (1.4.2), i.e. that the limit of Theorem 1.4 exists when one uses an alternating $j$-sum.

Section 5 is a generalization of the work of Olbrich [57] to the case of an arbitrary local system. In particular, we compute explicitly the constant $c_{G, M}$ of (1.4.2) and verify that it is positive. (We do not see a simple way to check this; we compute each case.)

In $\S 6$, we discuss the conjectures of Ash and their relationship to Theorem 1.4.

Section 7 has a different flavour from the rest of the paper; it studies a tower of coverings of an arbitrary cell complex. We show, in particular, that a suitable torsion quotient grows at a consistent rate as one passes through cyclic covers of a fixed base manifold, generalizing slightly the result of [65].

Section 8 details some instances to which Theorem 1.4 is applicable. In particular, strongly acyclic bundles always exist in the setting of Theorem 1.4 when $\delta=1$.

Finally, $\S 9$ outlines various questions and conjectures motivated by the rest of the paper. Several of these questions seem amenable to numerical study.

\section{Reidemeister torsion, torsion homology, and regularized determinants}

This section is expository: a recollection of works by Ray, Singer, Cheeger, and Müller, as well as some remarks related to $L^{2}$-torsion. We have attempted to present it in a way that motivates our main questions and theorems. The reader with some experience with torsion (analytic and Reidemeister) could likely skip this section.

In other words, we shall discuss:

(1) analytically computing homological torsion for a Riemannian manifold;

(2) limiting behaviour as the manifold grows (say, through a tower of covers).

We do not present the material in generality, but only adapted to our case of interest.

${ }^{5}$ Note that if $X$ is a compact manifold and $M$ a $\mathbb{Z}\left[\pi_{1} X\right]$-module free over $\mathbb{Z}$, Poincaré duality implies that $H^{*}(X ; M) \cong H_{\operatorname{dim} X-*}(X ; M)$. 


\section{1 .}

Given a finite rank free $\mathbb{Z}$-module $A$, such that $A \otimes \mathbb{R}$ is endowed with a positive definite inner product $\langle\cdot, \cdot\rangle$ (a metric for short), we define $\operatorname{vol}(A)$ to be the volume of $A \otimes \mathbb{R} / A$, i.e. $\operatorname{vol}(A)=\sqrt{|\operatorname{det} M|}$, where $M$ is the Gram matrix $\left\langle a_{i}, a_{j}\right\rangle$, for $a_{i}: 1 \leqslant i \leqslant \operatorname{rank}(A)$ a $\mathbb{Z}$-basis for $A$.

Given $f: A_{1} \rightarrow A_{2}$, a linear map between two finite rank free $\mathbb{Z}$-modules, such that $A_{j} \otimes \mathbb{R}$ are endowed with metrics, we set $\operatorname{det}^{\prime}(f)$ to be the product of all nonzero singular values of $f$. (Recall that the nonzero singular values of $f$ are - with multiplicity - the positive square roots of the nonzero eigenvalues of $f f^{*}$.) Then,

$$
\frac{\operatorname{vol}\left(A_{1}\right)}{\operatorname{vol}(\operatorname{ker} f)} \operatorname{det}^{\prime}(f)=\operatorname{vol}(\text { image } f)
$$

Here we understand the metrics on $\operatorname{ker}(f) \otimes \mathbb{R}$ and image $(f) \otimes \mathbb{R}$ as those induced from $A_{1}$ and $A_{2}$, respectively.

\section{2 .}

Now given a complex

$$
A^{*}: 0 \rightarrow A^{0} \stackrel{d_{0}}{\longrightarrow} A^{1} \stackrel{d_{1}}{\longrightarrow} \cdots \rightarrow A^{n} \rightarrow 0
$$

of free finite rank $\mathbb{Z}$-modules, each endowed with metrics and with $\operatorname{vol}\left(A^{j}\right)=1$, the previous result says that

$$
\operatorname{vol}\left(\operatorname{ker}\left(d_{j}\right)\right) \operatorname{vol}\left(\operatorname{image}\left(d_{j}\right)\right)=\operatorname{det}^{\prime}\left(d_{j}\right) .
$$

If the group $H^{j}\left(A^{*}\right)$ is finite, image $\left(d_{j-1}\right)_{\mathbb{R}}=\operatorname{ker}\left(d_{j}\right)_{\mathbb{R}}$ and the quotient $\frac{\operatorname{vol}\left(\operatorname{ker} d_{j}\right)}{\operatorname{vol}\left(\text { image } d_{j-1}\right)}$ is equal to $\left[\operatorname{ker}\left(d_{j}\right): \text { image }\left(d_{j-1}\right)\right]^{-1}$, i.e. the inverse of the size of the group $H^{j}\left(A^{*}\right)$. In general we write

$$
\operatorname{ker}\left(d_{j}\right)_{\mathbb{R}}=\text { image }\left(d_{j-1}\right)_{\mathbb{R}} \oplus \text { image }\left(d_{j-1}\right)^{\perp} \cap \operatorname{ker}\left(d_{j}\right)_{\mathbb{R}}
$$

and applying (2.1.1) to the orthogonal projection $\operatorname{ker} d_{j} \rightarrow$ image $\left(d_{j-1}\right)^{\perp}$ we conclude that the quotient $\frac{\operatorname{vol}\left(\operatorname{ker} d_{j}\right)}{\operatorname{vol}\left(\text { image } d_{j-1}\right)}$ is the product of $\left|H^{j}\left(A^{*}\right)_{\text {tors }}\right|^{-1}$ with the 'regulator' $R^{j}\left(A^{*}\right):=\operatorname{vol}\left(H^{j}\left(A^{*}\right)_{\text {free }}\right)$, where the subscript free denotes quotient by torsion, and where the volume is taken with respect to the induced metric - that is to say, the metric induced by identifying $H^{j}\left(A^{*}\right)_{\text {free }}$ as a subgroup of image $\left(d_{j-1}\right)^{\perp} \cap \operatorname{ker}\left(d_{j}\right)_{\mathbb{R}}$.

In summary,

$$
\begin{gathered}
\frac{\operatorname{vol}\left(\operatorname{ker} d_{j}\right)}{\operatorname{vol}\left(\operatorname{image} d_{j-1}\right)}=\left|H_{\text {tors }}^{j}\right|^{-1} \cdot R^{j} \\
H^{j}=H^{j}\left(A^{*}\right)=\frac{\operatorname{ker} d_{j}}{\operatorname{image~} d_{j-1}}, R^{j}=R^{j}\left(A^{*}\right):=\operatorname{vol}\left(H^{j}\left(A^{*}\right)_{\text {free }}\right) .
\end{gathered}
$$

To ease notation in what follows, we shall use the notation $\prod^{*} a_{i}$ as shorthand for $\prod_{i} a_{i}^{(-1)^{i}}$. In the same way we will use the notation $\sum^{*} a_{i}$ for the alternating sum $\sum(-1)^{i} a_{i}$. 
Taking the alternating product of (2.2.3),

$$
\left(\prod^{*} R^{i}\right) \times\left(\prod^{*}\left|H_{\mathrm{tors}}^{i}\right|\right)^{-1}=\prod^{*} \operatorname{det}^{\prime}\left(d_{i}\right)
$$

Example. Consider the complex of free $\mathbb{Z}$-modules, where the first nonvanishing term is in degree 0:

$$
0 \rightarrow \mathbb{Z}^{2} \stackrel{M}{\rightarrow} \mathbb{Z}^{2} \rightarrow 0
$$

where

$$
M=\left(\begin{array}{cc}
k^{2} & -k \\
-k & 1
\end{array}\right) \quad(k \in \mathbb{Z}) .
$$

Then $H^{0} \cong H^{1} \cong \mathbb{Z}$; and then $R_{0}=\sqrt{k^{2}+1}, R_{1}=\frac{1}{\sqrt{k^{2}+1}} ;$ the singular values of $M$ are 0 and $k^{2}+1$.

The alternating product $\prod^{*} \operatorname{det}^{\prime}\left(d_{i}\right)$ is, in many ways, unwieldy: the $d_{i}$ may have large kernels even if the complex is acyclic. On the other hand, the identity

$$
\left(\prod^{*}\left(\operatorname{det}^{\prime} d_{i}\right)\right)^{2}=\prod_{i \geqslant 0}\left(\operatorname{det}^{\prime} \Delta_{i}\right)^{i(-1)^{i+1}}, \Delta:=d d^{*}+d^{*} d
$$

(see [60, Proposition 1.7]) - here $\operatorname{det}^{\prime}$ is the product of all nonzero eigenvalues, and this usage is compatible with our prior one - expresses it in terms of the determinants of the 'Laplacians' $\Delta_{i}$, whose kernels map isomorphically to cohomology (and in particular, are much smaller than $\left.\operatorname{ker}\left(d_{i}\right)\right)$.

\section{3 .}

Let $X$ be a compact Riemannian manifold and $M$ a unimodular local system of free $\mathbb{Z}$-modules on $X$. Let $M_{\mathbb{C}}=M \otimes \mathbb{C}$.

Fix a $C^{\infty}$ triangulation of $K$. Let $C^{q}(K ; M)$ be the set of $M$-valued cochains, so that an element of $C^{q}(K ; M)$ assigns to each $q$-cell a section of $M$ on that cell. Then we have a cochain complex:

$$
C^{*}(K ; M): C^{0}(K ; M) \rightarrow C^{1}(K ; M) \rightarrow \cdots
$$

which computes the cohomology groups $H^{*}(K ; M)=H^{*}(X ; M)$. The de Rham complex

$$
\Omega^{*}(X ; M): \Omega^{0}\left(X ; M_{\mathbb{C}}\right) \stackrel{d_{0}^{D R}}{\longrightarrow} \Omega^{1}\left(X ; M_{\mathbb{C}}\right) \rightarrow \cdots
$$

computes also $H^{*}\left(X ; M_{\mathbb{C}}\right)$. It can be regarded as a 'limit' of the prior complex when the triangulation becomes very fine. We fix arbitrarily a metric on $M_{\mathbb{C}}$; then each term of the complex $\Omega^{*}$ is equipped with a natural inner product.

The amazing discovery of Ray and Singer (as proved by Cheeger and Müller [21,54] for trivial coefficients (or more generally for all unitary representations), and later by Müller [55] for general unimodular $M$ ) is that the equality (2.2.4), when applied 
to $C^{*}(K ; M)$, 'passes to the limit' in the following sense:

$$
\left(\prod^{*} R^{i}\right) \times\left(\prod^{*}\left|H^{i}(X ; M)_{\mathrm{tors}}\right|^{-1}\right)=\prod^{*} \operatorname{det}^{\prime} d_{i}^{D R},
$$

where $R^{i}$ is now defined as the volume of $H^{i}(X ; M) / H^{i}(X ; M)_{\text {tors }}$ with respect to the metric induced by identifying cohomology with harmonic forms. To make sense of $\operatorname{det}^{\prime} d_{i}^{D R}$, write $\zeta(s)=\sum \lambda^{-s}$, the sum being extended over all nonzero singular values of $d_{i}^{D R}$; we then define $\log \operatorname{det}^{\prime} d_{i}^{D R}:=-\zeta^{\prime}(0)$ and $\operatorname{det}^{\prime} d_{i}^{D R}=\exp \left(-\zeta^{\prime}(0)\right)$. Note that $\zeta(s)$ is defined near zero only after analytic continuation.

More generally, for any countable subset $S$ of positive reals such that $\sum_{\lambda \in S} \lambda^{-s}$ extends to a meromorphic function in $\operatorname{Re}(s) \geqslant 0$, we refer to the outcome of this procedure as the ' $\zeta$-regularized product', denoted as $\prod_{\lambda \in S}^{\wedge} \lambda$.

This is usually formulated slightly differently. Let $d_{i}^{*}$ denote the adjoint of the differential $d_{i}=d_{i}^{D R}$. Define the Laplace operators on $i$-forms by $\Delta_{i}=d_{i}^{*} d_{i}+d_{i-1} d_{i-1}^{*}$. Again, one can define the logarithmic determinant of $\Delta_{i}$ as the zeta-regularized product of nonzero eigenvalues.

Then we have the following, easily verified, identity:

$$
\sum^{*} \log \operatorname{det}^{\prime} d_{i}=\frac{1}{2} \sum_{k \geqslant 0}(-1)^{k+1} k \log \operatorname{det}^{\prime} \Delta_{k} .
$$

Ray and Singer have defined the logarithm of the analytic torsion of $(X, M)$ to be the negative of the right-hand side of (2.3.3).

In summary, the Cheeger-Müller theorem states

$$
\begin{aligned}
\log \frac{\left(\prod^{*} R^{i}\right)}{\left(\prod^{*}\left|H^{i}(X ; M)_{\text {tors }}\right|\right)} & =\frac{1}{2} \sum_{k \geqslant 0}(-1)^{k+1} k \log \operatorname{det}^{\prime} \Delta_{k} \\
& =\log T_{X}\left(M_{\mathbb{C}}\right) \quad \text { (analytic torsion) } .
\end{aligned}
$$

which has the remarkable consequence that torsion in homology groups can be studied by analytical methods.

\section{4 .}

Consider a simple example: $X=S^{1}=\mathbb{R} / \mathbb{Z}$, endowed with the quotient of the standard metric on $\mathbb{R}$; let $M$ be a free rank $m \mathbb{Z}$-module, $A \in \mathrm{SL}(M)$, and $\mathcal{M}$ the local system on $X$ with fibre $M$ and monodromy $A$.

Suppose first that $A$ is semisimple and does not admit 1 as an eigenvalue. (The situation where $A$ has 1 as an eigenvalue is interesting; we return to it in $\S 2.6$.) The cohomology is then concentrated in $H^{1}$; moreover

$$
\left|H^{1}(X, \mathcal{M})\right|=|\operatorname{det}(1-A)| \text {. }
$$

Let us now compute the de Rham complex. Let $V_{j}$ be the space of $M_{\mathbb{C}}:=M \otimes \mathbb{C}$-valued $j$-forms on $S^{1}$, for $j=0,1$. Let

$$
S=\left\{\text { smooth functions } f: \mathbb{R} \rightarrow M_{\mathbb{C}}: f(x+1)=A f(x)\right\}
$$


and

$$
T=\left\{\text { smooth functions } f: \mathbb{R} \rightarrow M_{\mathbb{C}}: f(x+1)=f(x)\right\} .
$$

We may identify each $V_{j}$ with $S$ : this is obvious for $j=0$; use the map $f(x) \mapsto f(x) d x$ for $j=1$. Next, fix a matrix $B \in \mathrm{GL}\left(M_{\mathbb{C}}\right)$ with $\exp (2 \pi i B)=A$; this is always possible, and then multiplication by $\exp (-2 \pi i x B)$ identifies $S$ with the space $T$.

Thus we have identifications of $V_{j}$ with $T$ for $j=0,1$. With respect to these identifications, the de Rham complex becomes

$$
T \stackrel{d}{\rightarrow} T \quad \text { with } d=\frac{d}{d x}+2 \pi i B .
$$

Fix a basis of eigenvectors $v_{1}, \ldots, v_{m}$ for $B$ on $M_{\mathbb{C}}$, and endow $M_{\mathbb{C}}$ with the inner product in which the $v_{i}$ form an orthonormal basis; endow $T$ with the inner product $\langle f, g\rangle=$ $\int_{x \in \mathbb{R} / \mathbb{Z}}\langle f(x), g(x)\rangle$. Then the singular values of $d$ are $\left|2 \pi i\left(n+\lambda_{j}\right)\right|, n \in \mathbb{Z}, \quad 1 \leqslant j \leqslant m$, where $\lambda_{j}$ are the eigenvalues of $B$. Formally speaking, then, the product of singular values of $d$ may be expressed as $\prod_{n} \prod_{j}\left|2 \pi n+2 \pi \lambda_{j}\right|$. If we compute formally, using the identity $\prod_{n \geqslant 1}\left(1-\frac{x^{2}}{n^{2}}\right)=\frac{\sin (\pi x)}{\pi x}$, we arrive at

$$
\left|\operatorname{det}^{\prime} d_{D R}\right|=|\operatorname{det}(1-A)|\left(\prod_{n \geqslant 1}(2 \pi n)\right)^{2} .
$$

Now $\prod_{n \geqslant 1} 2 \pi n=1$ (a zeta-regularized product), as desired. In fact, $\zeta_{\lambda}(s)=(2 \pi)^{-s} \zeta(s)$ with $\zeta$ the Riemann zeta function. Because $\zeta^{\prime}(0)=-\frac{1}{2} \log (2 \pi)$ and $\zeta(0)=-\frac{1}{2}$, we get

$$
\prod_{n \geqslant 1}^{\wedge}(2 \pi n)=1 \text {. }
$$

Remark. On the basis of the naive (but natural) idea of considering the asymptotics of $\prod_{\lambda_{j} \leqslant T} \lambda_{j}$, one can also give an alternate definition of the regularized product: let $h$ be a smooth function on $\mathbb{R}$ of compact support such that $h(0)=1$. Set $P(T):=$ $\sum_{\lambda_{i}} h\left(\lambda_{i} / T\right) \log \lambda_{i}$. Under reasonable conditions on the $\lambda_{i}$ (true in all the instances that we will encounter, for instance) there exists a unique polynomial $q \in \mathbb{C}[X, Y]$ such that

$$
P(T)=q(T, \log T)+o(1), \quad T \rightarrow \infty,
$$

and we define $\prod^{\wedge} \lambda_{i}=\exp (q(0,0))$. This will coincide with $\zeta$-regularization in the cases that we encounter.

This definition is not the traditional one, but, in most instances, is equivalent to it. It has the advantage of being very intuitive, but, on the other hand, it is difficult to work with. Since we will not use it for rigorous proofs, only for intuition, we shall not prove that it agrees with the zeta-regularized definition.

In the case of the $(2 \pi n)_{n \in \mathbb{N}}$, the fact that $\prod^{\wedge} 2 \pi n=1$ 'corresponds' in this picture to the fact that there is no constant term in the asymptotics of Stirling's formula:

$$
\log \prod_{n \leqslant N-1}(2 \pi n) \cdot \sqrt{2 \pi N} \sim N \log N-N ;
$$

we included the endpoint $n=N$ with weight $1 / 2$, a simple form of smoothing. 


\section{5.}

We continue with the example of $\S 2.4$, and let $X \stackrel{\pi_{N}}{\longrightarrow} X$ be the $N$-fold covering. If we suppose that $A$ has no eigenvalue that is a root of unity, then

$$
\frac{\log \left|H^{1}\left(X, \pi_{N}^{*} \mathcal{M}\right)\right|}{N}=\frac{\log \left|\operatorname{det}\left(1-A^{N}\right)\right|}{N} \longrightarrow \log M(\operatorname{charpoly}(A)),
$$

where the Mahler measure $M(q)$ of a monic polynomial $q$ is the product of all the absolute values of all roots of $q$ outside the unit circle; equivalently, $\log M(q)=$ $\int_{|z|=1} \log |q(z)| \frac{d z}{2 \pi i z}$ (the integral taken with respect to the Haar probability measure) as follows from Jensen's equality. We shall later return to the case where $A$ has roots of unity; we shall find that a similar assertion remains valid.

The proof of (2.5.1) uses the following lemma.

Lemma. If $A$ is any square matrix,

$$
\lim _{N} \frac{\log \left|\operatorname{det}^{\prime}\left(1-A^{N}\right)\right|}{N}=\log M(\operatorname{charpoly}(A)),
$$

where det' $^{\prime}$ denotes the product of all nonzero eigenvalues.

Proof. The first proof in print of (2.5.2) seems to be by Lind [45] in the context of a dynamical interpretation. The proof uses the following fact (due to Gelfond [31]): if $\alpha$ is an algebraic number of absolute value 1 , then

$$
\frac{\log \left|\alpha^{N}-1\right|}{N} \rightarrow 0, \quad N \rightarrow \infty .
$$

We refer the reader to [29, Lemma 1.10] for a proof of (2.5.3) which uses a deep theorem of Baker on diophantine approximation. As noticed there, the statement (2.5.3) is much weaker than Baker's theorem; it is exactly equivalent to Gelfond's earlier estimate.

\subsection{The case where $A$ has a trivial eigenvalue}

Now let us repeat the procedure of $\S 2.5$ for the case where $A$ has a trivial eigenvalue. We suppose, for simplicity, that precisely one eigenvalue of $A$ is 1 , and amongst the other eigenvalues $\left\{z_{2}, \ldots, z_{n}\right\}$ none is a root of unity. We are interested in the size of $H^{1}\left(X, \pi_{N}^{*} \mathcal{M}\right) \cong \operatorname{coker}\left(1-A^{N}\right)_{\text {tors }}$, as $N \rightarrow \infty$.

Write $W=\operatorname{ker}(1-A)$ and $U=\operatorname{image}(1-A)$; finally put $C=\left.\frac{1-A^{N}}{1-A}\right|_{U}$. Noting that $U / C U \subset \mathbb{Z}^{N} /\left(1-A^{N}\right) \mathbb{Z}^{N}$, we see at least that

$$
\left|\operatorname{coker}\left(1-A^{N}\right)_{\text {tors }}\right| \geqslant|\operatorname{det} C|=\prod_{2}^{n} \frac{z_{i}^{N}-1}{z_{i}-1} .
$$

Consider now the short exact sequence $W \hookrightarrow \mathbb{Z}^{n} \rightarrow \mathbb{Z}^{n} / W$. The map $1-A^{N}$ acts trivially on $W$, and descends to an endomorphism $A^{\prime}$ of $\mathbb{Z}^{N} / W$. Applying the snake lemma yields an exact sequence:

$$
0 \rightarrow \mathbb{Z} \rightarrow \operatorname{coker}\left(1-A^{N}\right) \rightarrow \operatorname{coker}\left(1-A^{\prime N}\right) \rightarrow 0,
$$

which implies the upper bound $\prod_{2}^{n}\left(z_{i}^{N}-1\right)$. 
We deduce that (2.5.1) remains valid even if $A$ has 1 as an eigenvalue, i.e., even in the case where $H^{1}\left(S^{1}, \mathcal{M}\right)$ is not a torsion group.

In general, the question of extending our results to the case where the homology is not purely torsion will be very difficult; the 'interaction' between characteristic 0 cohomology and torsion can be very complicated. Implicitly in the above argument, we used the possibility of 'splitting' into $\operatorname{ker}(1-A)$ and image $(1-A)$; more generally, we can carry out such a 'splitting' in the presence of a group of automorphisms, but not in the general setting. We discuss some of the issues involved (including extending the discussion above to a more general setting - that of cyclic covers of an arbitrary base complex) in $\S 7$.

\section{7 .}

Let us now try to prove (2.5.1) from the 'de Rham' perspective, returning to the case where $A$ has no eigenvalue that is a root of unity.

If we carry out the same computations as before for the determinant of the de Rham complex of $\pi_{N}^{*} \mathcal{M}$ - with respect to metrics pulled back from $X$ - we arrive at

$$
\left|H^{1}\left(X, \pi_{N}^{*} \mathcal{M}\right)_{\text {tors }}\right|=\prod_{n \in \mathbb{Z}} \prod_{j}^{\wedge}\left|\frac{2 \pi i n}{N}+2 \pi i \lambda_{j}\right| .
$$

Now as $N \rightarrow \infty$, the sequence of numbers $\frac{2 \pi n}{N}+2 \pi \lambda_{j}$ fill out densely the line $2 \pi \lambda_{j}+\mathbb{R}$; the 'density' is $N / 2 \pi$. It is therefore reasonable to imagine that it converges to a regularized integral:

$$
\frac{\log \prod_{n \in \mathbb{Z}^{\wedge}}\left|\frac{2 \pi i n}{N}+2 \pi i \lambda_{j}\right|}{N / 2 \pi} \stackrel{?}{\longrightarrow} \int^{\wedge} \log \left|2 \pi \lambda_{j}+x\right| d x,
$$

where the wedge on the latter integral means that it is to be understood, again, in a regularized fashion: we may define, analogously to $\zeta$-regularization, the regularized integral via

$$
\int^{\wedge} \log |f(x)| d x:=-\left.\frac{d}{d s}\right|_{s=0} \int|f(x)|^{-s} d x .
$$

We evaluate the right-hand side of (2.7.1) (and a slight generalization) in $\S 2.8$ below; it is $2 \pi^{2}\left|\operatorname{Im} \lambda_{j}\right|$. Therefore, if we assume the validity of (2.7.1),

$$
\frac{\log \left|H^{1}\left(X, \pi_{N}^{*} \mathcal{M}\right)_{\text {tors }}\right|}{N} \longrightarrow \pi \sum_{j}\left|\operatorname{Im}\left(\lambda_{j}\right)\right|=2 \pi \sum_{\operatorname{Im}\left(\lambda_{j}\right)>0} \operatorname{Im}\left(\lambda_{j}\right),
$$

which is easily seen to again equal $\log M(\operatorname{charpoly}(A))$.

The validity of (2.7.1) is, however, not easy to justify in general, as perhaps might be expected from the nontriviality of (2.5.3). The issue here is that some $\lambda_{j}$ may have imaginary part 0 (equivalently: $A$ has an eigenvalue of absolute value 1 ). Suppose that this is not so. Then (2.7.1) can be deduced, for instance, from the 'naive definition' (2.4.2) of regularized products. The key point is that the convergence of 
the sequence $\frac{2 \pi n}{N}$ to the density $\frac{N}{2 \pi} \mathrm{d} x$ is uniform, in that

$$
\#\{n: 2 \pi n / N \in[A, B]\}-\int_{A}^{B} \frac{\mathrm{d} x}{2 \pi}=\mathrm{o}(N),
$$

in a fashion that is uniform in $A, B$.

In the rest of this paper, we shall encounter a similar, although more complicated, situation: we will compute the asymptotic growth of $H^{1}$ by studying the asymptotic behaviour of the de Rham determinants. It would be possible to justify our main theorems in the fashion described above - in other words, deducing the limiting behaviour of torsion from the limiting distribution of eigenvalues. However, for brevity of proof we follow a more traditional path with heat kernels; this allows us to appeal to a family of known estimates at various points.

\section{8 .}

Let $a>0$ be a positive real number and $p$ be an even polynomial. We show that

$$
\int^{\wedge} p(\mathrm{i} x) \log \left(x^{2}+a^{2}\right) d x=\pi \int_{-a}^{a} p(x) d x,
$$

where the left-hand side is defined as in (2.7.2), but replacing the Lebesgue measure by $p(\mathrm{i} x) d x$.

Proof. By linearity, it suffices to check $p(x)=x^{k}$ with $k$ even. In this case, ${ }^{6}$

$$
\int_{-\infty}^{\infty}\left(x^{2}+a^{2}\right)^{-s} x^{k} d x=a^{2\left(\frac{k+1}{2}-s\right)} B\left(\frac{k+1}{2}, s-\frac{k+1}{2}\right) .
$$

Here $B$ is the Beta function

$$
B(x, y)=\int_{0}^{1} t^{x-1}(1-t)^{y-1} d t=\frac{\Gamma(x) \Gamma(y)}{\Gamma(x+y)} .
$$

Differentiating the function

$$
a^{2\left(\frac{k+1}{2}-s\right)} \frac{\Gamma\left(\frac{k+1}{2}\right) \Gamma\left(s-\frac{k+1}{2}\right)}{\Gamma(s)}=a^{2\left(\frac{k+1}{2}-s\right)} s \frac{\Gamma\left(\frac{k+1}{2}\right) \Gamma\left(s-\frac{k+1}{2}\right)}{\Gamma(s+1)}
$$

at $s=0$ and using that

$$
\Gamma\left(\frac{k+1}{2}\right) \Gamma\left(-\frac{k+1}{2}\right)=(-1)^{k / 2+1} \frac{2 \pi}{k+1}
$$

yields $-i^{k} \frac{2 \pi a^{k+1}}{k+1}$.

Again, this reflects the constant term of a truncated integral, e.g. we observe, in the case $p=1$, that $\int_{-T}^{T} d x \log \left(1+x^{2}\right)=4 T \log (T)-4 T+2 \pi+o(1)$.

6 Take as a new variable $t=x^{2} /\left(x^{2}+a^{2}\right)$. 


\section{Heat kernels on forms on a symmetric space}

\section{1 .}

Let $\mathbf{G}$ be a semisimple algebraic group over $\mathbb{R}$; let $G$ be the connected component of $\mathbf{G}(\mathbb{R})$, with Lie algebra $\mathfrak{g}$.

Let $K \subset G$ be a maximal compact subgroup, with Lie algebra $\mathfrak{k}$. Since $G$ is connected, so also is $K$. We denote by $S$ the global Riemannian symmetric space $G / K$.

Remark (Notation for real and complex Lie algebras.). When we perform explicit evaluations in $\S 5$, we shall use the letters $\mathfrak{g}, \mathfrak{k}$ and so on to denote the complexified Lie algebra associated with $G, K$ and so on. However, in the present section, we use them for the real Lie algebra; we hope that this abuse does not cause confusion.

\section{2 .}

With a $K$-representation $(Q, \sigma)$, we may associate a $G$-equivariant vector bundle on $G / K$, namely, $(Q \times G) / K$, where the $K$-action (resp. $G$-action) is given by $(q, g) \stackrel{k}{\longrightarrow}$ $\left(\sigma\left(k^{-1}\right) q, g k\right)$ (resp. $\left.(q, g) \stackrel{x}{\rightarrow}(q, x g)\right)$; thus, smooth sections of the $G$-equivariant vector bundle associated with $Q$ are identified with maps $C^{\infty}(G ; Q)$ with the property that $f(g k)=\sigma\left(k^{-1}\right) f(g)$.

Conversely, given a $G$-equivariant vector bundle on $G / K$, the fibre at the identity coset defines a $K$ representation.

These two associations define an equivalence of categories between $K$-representations and $G$-equivariant vector bundles on $S$.

\section{3.}

We now discuss normalizations.

The Killing form defines a quadratic form on $\mathfrak{g}$, negative definite on $\mathfrak{k}$ and positive definite on its orthogonal complement $\mathfrak{p}$. We normalize the metric on $S$ so that its restriction to $\mathfrak{p} \cong T_{e K} S$ matches the Killing form.

Let $d x$ be the Riemannian volume form of $S$ and $d k$ be the Haar measure on $K$ of volume 1 . These choices yield a preferred normalization for the Haar measure $d g$ on $G$ : $\int_{G} f(g) d g=\int_{S} \int_{K} f(g k) d k d(g K)$.

Fix orthonormal bases $\left\{X_{i}\right\}$ for $\mathfrak{p}$ and $\left\{Y_{j}\right\}$ for $\mathfrak{k}$ (the latter taken with respect to the negative of the Killing form). We define the Casimir operators of $G$ and $K$ respectively by $\Omega_{G}=\sum X_{i}^{2}-\sum Y_{j}^{2}$ and $\Omega_{K}=-\sum Y_{j}^{2}$; we understand these to act on $G$ and $K$ as left-invariant differential operators. Let

$$
L=-\Omega_{G}+2 \Omega_{K} \in \mathcal{U}(\mathfrak{g}) .
$$

The operator $L$ on $G$ is the infinitesimal generator of a convolution semigroup of absolutely continuous measures on the group $G$. Indeed, the right action of $L$ coincides with the Laplacian with respect to a suitable metric on $G$.

There exists, for positive $t$, a function $p_{t}: G \rightarrow \mathbb{R}_{\geqslant 0}$ such that $\mathrm{e}^{-t L} f=f * p_{t}$, i.e., the function $g \mapsto \int_{h \in G} f(g h) p_{t}(h) \mathrm{d} h$. In fact, on any $G$-representation, the action of $p_{t}$ by convolution coincides with the action of $\mathrm{e}^{-t L}$. 


\section{4 .}

Put $\mathfrak{u}=\mathfrak{k} \oplus i \mathfrak{p}$; its normalizer inside $G_{\mathbb{C}}:=\mathbf{G}(\mathbb{C})$ is a maximal compact subgroup $U$ of $G_{\mathbb{C}}$. Let $\rho$ be an irreducible representation of $U$; it extends to a unique holomorphic representation of $G_{\mathbb{C}}$ on a complex vector space $E_{\rho}$, or indeed an algebraic representation of $\mathbf{G}$. Since $\mathbf{G}$ is semisimple, the representation $\rho$ is necessarily unimodular, i.e. takes values in $\operatorname{SL}\left(E_{\rho}\right)$.

There is, up to scaling, one $U$-invariant Hermitian metric on $E_{\rho}$. We fix an inner product $(-,-)_{E}$ in this class.

The representation $\rho \mid K$ gives rise to a $G$-equivariant Hermitian vector bundle on $G / K$, as in $\S 3.2$. We denote this vector bundle also as $E_{\rho}$. Note that this bundle is $G$-equivariantly isomorphic to the trivial vector bundle $E_{\rho} \times G / K$, where the $G$-action is via $x:(e, g K) \longrightarrow(\rho(x) e, x g K)$.

\section{5.}

The bundle of $E_{\rho}$-valued differential $k$-forms on $S$ can be identified with the vector bundle associated (via $\S 3.2$ ) with the $K$-representation $\bigwedge^{k} \mathfrak{p}^{*} \otimes E$.

Note that $\bigwedge^{k} \mathfrak{p}^{*} \otimes E$ is naturally endowed with a $K$-invariant scalar product: the tensor product of $(,)_{E}$ with the scalar product on $\bigwedge^{k} \mathfrak{p}^{*}$ defined by the Riemannian metric on $S$. The space of differentiable $E$-valued $k$-forms on $S$, denoted as $\Omega^{k}(S, E)$, is therefore identified with

$$
\left.\left\{\varphi: G \stackrel{C^{\infty}}{\longrightarrow} \bigwedge^{k} \mathfrak{p}^{*} \otimes E: \varphi(g k)=\bigwedge^{k} \operatorname{ad}_{\mathfrak{p}}^{*}\left(k^{-1}\right) \otimes \rho\left(k^{-1}\right)\right)(\varphi(g)), \quad g \in G, k \in K\right\} .
$$

The space of square integrable $k$-forms $\Omega_{(2)}^{k}(S, E)$ is the completion of the subspace which consists of the compactly supported $\varphi$ in the latter space with respect to the norm

$$
\varphi \mapsto \int_{S}\|\varphi(x K)\|_{\bigwedge^{k}{ }^{*}{ }^{*} \otimes E}^{2} d x
$$

\subsection{The de Rham complex}

Let $V$ be any $(\mathfrak{g}, K)$-module equipped with a $K$-invariant Hermitian form; we do not require this form to be $G$-invariant. We will be particularly interested in $V_{0}=C_{c}^{\infty}(G)_{K} \otimes E$, where $C_{c}^{\infty}(G)_{K}$ denotes right $K$-finite smooth compactly supported functions.

Set

$$
D^{k}(V)=\bigwedge^{k} \mathfrak{p}^{*} \otimes V
$$

We introduce the natural inner product on $D^{k}(V)$. Thus, for instance, $D^{k}\left(V_{0}\right)^{K}$ is identified with the space $\Omega^{k}(S, E)$ of $E$-valued $k$-forms.

Generalizing the differential on $E$-valued $k$-forms, one may define for any $V$ a natural differential $d: D^{k}(V) \rightarrow D^{k+1}(V)$; see [10, II. Proposition 2.3]. In the case $V=V_{0}$, its restriction to $D^{k}\left(V_{0}\right)^{K}$ recovers the de Rham differential. Let $d^{*}$ be the formal adjoint of $d$. We refer to the restriction of $d d^{*}+d^{*} d$ to $D^{k}(V)^{K}$ as the Laplacian. 
In the case $V=V_{0}$, this 'Laplacian' extends to a $G$-invariant self-adjoint nonnegative densely defined elliptic operator $\Delta_{k}^{(2)}$ on $\Omega_{(2)}^{k}(S, E)$, the form Laplacian. ${ }^{7}$

In general, the Laplacian is difficult to compute. However, the fact that the bundle $E_{\rho}$ arose from the restriction of a $G$-representation makes the situation simpler: if the Casimir acts on $V$ (resp. $E$ ) through the scalar $\Lambda_{V}$ (resp. $\Lambda_{\rho}$ ), Kuga's lemma implies that for any $f \in D^{k}(V)^{K}$,

$$
\left(d^{*} d+d d^{*}\right) f=\left(\Lambda_{\rho}-\Lambda_{V}\right) f .
$$

In other terms, the Laplacian acts by the same scalar on the entire complex $D^{*}(V)^{K}$.

Of course, this does not literally apply to our case of primary interest, when $V=V_{0}$, for the Casimir does not act on $V_{0}$ as a scalar. However, one may decompose $V_{0}$ into irreducible subrepresentations and then apply (3.6.1).

\section{7.}

Denote by $\mathrm{e}^{-t \Delta_{k}^{(2)}} \in \operatorname{End}\left(\Omega_{(2)}^{k}(S, E)\right)$ the bounded operator (cf. [4]) defined by the fundamental solution of the heat equation:

$$
\left\{\begin{array}{l}
\Delta_{k}^{(2)} P_{t}=-\frac{\partial}{\partial t} P_{t}, \quad t>0 \\
P_{0}=\delta
\end{array}\right.
$$

where $\delta$ is the Dirac distribution.

It is expressed by an integral kernel (the heat kernel) $\mathrm{e}^{-t \Delta_{k}^{(2)}}$, which we may regard as a section of a certain bundle $\mathscr{E}_{k}$ over $S \times S$. Explicitly, this is the $G \times G$-equivariant bundle associated with the $K \times K$-representation $\operatorname{End}\left(\bigwedge^{k} \mathfrak{p}^{*} \otimes E, \bigwedge^{k} \mathfrak{p}^{*} \otimes E\right)$; more explicitly, the fibre of $\mathscr{E}_{k}$ above $(x, y)$ is the set of homomorphisms from $E$-valued $k$-forms at $x$ to $E$-valued $k$-forms at $y$. Moreover,

$$
\left(\mathrm{e}^{-t \Delta_{k}^{(2)}} f\right)(x)=\int_{S} \mathrm{e}^{-t \Delta_{k}^{(2)}}(x, y) f(y) d y, \quad \forall f \in \Omega_{(2)}^{k}(S, E) .
$$

Lemma 3.8. Let $M \geqslant 1$. Then there exists a constant $c_{1}$ depending only on $G, \rho, M$ such that

$$
\left\|\mathrm{e}^{-t \Delta_{k}^{(2)}}(x, y)\right\| \leqslant c_{1} t^{-d / 2} \exp \left(-\frac{r^{2}}{5 t}\right), \quad|t| \leqslant M,
$$

where $x, y \in S, r$ is the geodesic distance between $x$ and $y, d$ the dimension of $S$ and $\|\cdot\|$ is the metric induced by that on $E$.

The lemma gives us only information about the 'small time' dependence of the heat kernel. The 'long time' dependence is more sensitive; for instance, it is quite different if there exist $L^{2}$-harmonic forms (e.g., for $\left.G=\mathrm{SL}_{2}(\mathbb{R})\right)$.

Proof. First we make an observation in a more abstract context: if $(V, \pi)$ is any $G$-representation with Casimir eigenvalue $\Lambda_{\pi}$ and $(W, \sigma)$ any $K$-representation, the

${ }^{7}$ It should be noted that there is another notion of Laplacian, which is the specialization to $\bigwedge^{k} \mathfrak{p}^{*} \otimes E$ of the Laplacian that exists on any Hermitian bundle with connection. 
operator $Q:=\int_{k \in K} \pi(k) \otimes \sigma(k) d k$ realizes the projection of $V \otimes W$ onto $K$-invariants $(V \otimes W)^{K}$; on the other hand, denoting by $E_{t} \in \operatorname{End}(W)$ the operator $e^{t \Omega_{K}}$, we have for $t>0$ that

$$
T=\pi\left(p_{t}\right) \otimes E_{2 t}=\int_{G}\left(p_{t}(g) \pi(g)\right) \otimes E_{2 t} d g
$$

acts on $(V \otimes W)^{K}$ through $e^{t \Lambda_{\pi}}$. (Here $p_{t}$ is as in $\S 3.3$.) Thus $Q \cdot T \cdot Q$ certainly acts on $(V \otimes W)^{K}$ through the scalar $e^{t \Lambda_{\pi}}$.

On the other hand, expanding the expressions above, we see that $Q \cdot T \cdot Q=\pi\left(\psi_{t}\right)$ where $\psi_{t}$ is the $\operatorname{End}(W)$-valued function on $G$ given by

$$
\psi_{t}: g \mapsto \int_{K \times K} p_{t}\left(k_{1}^{-1} g k_{2}^{-1}\right)\left(\sigma\left(k_{1}\right) \cdot E_{2 t} \cdot \sigma\left(k_{2}\right)\right) d k_{1} d k_{2} .
$$

Let us note that the norm of the right-hand side, with respect to the natural Hilbert norm on $\operatorname{End}(W)$, is bounded above by a constant multiple (depending on $M, \sigma$ ) of $\int_{K \times K} p_{t}\left(k_{1}^{-1} g k_{2}^{-1}\right) d k_{1} d k_{2}$, since $p_{t}$ is positive. The integral $\int_{K \times K} p_{t}\left(k_{1}^{-1} g k_{2}^{-1}\right) d k_{1} d k_{2}$ defines a bi- $K$-invariant function on $G$ that is identified with the (usual) heat kernel on the symmetric space $S$; in particular, by the Cheng-Li-Yau bounds [22], it is bounded for $|t| \leqslant M$ by $c_{1}(M) t^{-d / 2} \exp \left(-\frac{r^{2}}{5 t}\right)$, with $r$ the distance between $g K$ and the identity coset.

We now apply this construction with $V$ equal to the underlying $(\mathfrak{g}, K)$-module of the right regular representation $L^{2}(G)$; and $W=\bigwedge^{k} \mathfrak{p}^{*} \otimes E$. In this case $V$ is no longer irreducible; however, breaking it into irreducibles and applying (3.6.1), we see that the operator (3.8.1) acts on $(V \otimes W)^{K}=D_{k}(V)^{K}$ in the same way as $\mathrm{e}^{-t \Delta_{k}^{(2)}} e^{t \Lambda_{\rho}}$, where $\Lambda_{\rho}=\rho(\Omega)$ is the Casimir eigenvalue of the $G$-representation $E$.

In other words, if we identify elements in $\Omega^{k}$ with functions on $G$ as specified in $\S 3.5$, we have

$$
\left(\mathrm{e}^{-t \Delta_{k}^{(2)}} f\right)(g)=\mathrm{e}^{-t \Lambda_{\rho}} \int_{G} \psi_{t}\left(g^{-1} g^{\prime}\right) f\left(g^{\prime}\right) d g^{\prime}, \quad \forall f \in \Omega_{(2)}^{k}(S, E) .
$$

Passing to the associated section of $\mathcal{E}_{k}$ gives the desired assertion.

\section{A 'limit multiplicity formula' for torsion}

We continue with the notation of the previous section; let $\Gamma$ be a cocompact torsion-free subgroup of $G$ and let $X=\Gamma \backslash S$. Set $V$ to be the vector bundle on $X$ induced by $\rho$ (i.e., the quotient of the total space $E_{\rho} \times S$ by the $\Gamma$-action). Let $\Delta_{k}$ be the Laplacian on $E_{\rho}$-valued $k$-forms on $X$.

Note that $H^{*}(X ; V)$ is isomorphic to $H^{*}(\Gamma ; V)$. We say, accordingly, that $\rho$ is acyclic for $\Gamma$ when $H^{k}(X ; V)=0$ for each $k$; equivalently, the smallest eigenvalue of each $\Delta_{k}$ is positive. We say that $\rho$ is strongly acyclic if there exists some uniform positive constant $\eta=\eta(G)>0$ such that every eigenvalue of every $\Delta_{k}$ for any choice of $\Gamma$ is $\geqslant \eta$. The remarkable fact is that there exists a large and interesting supply of strongly acyclic representations; see $\S 8$. To check this, the following will be necessary. 
Lemma 4.1. Suppose that the isomorphism class of $d \rho: \mathfrak{g} \rightarrow \mathfrak{g l}\left(E_{\rho}\right)$ is not preserved under the Cartan involution. Then $\rho$ is strongly acyclic.

Proof. Set $F=E_{\rho}^{*}$; it is a finite dimensional representation of $\mathfrak{g}$. It is known $[10, \S \mathrm{VII}$, Theorem 6.7] that, if the isomorphism class of $F$ is not fixed by the Cartan involution, then $\rho$ is acyclic (for any $\Gamma$ as above). In fact, one of the arguments explained there shows strong acyclicity, as we now explain.

First note that using

$$
\Omega_{(2)}^{*}\left(\Gamma \backslash S, E_{\rho}\right) \cong \operatorname{Hom}_{K}\left(\wedge^{*} \mathfrak{p} \otimes F, L^{2}(\Gamma \backslash G)\right)
$$

and the decomposition of $L^{2}(\Gamma \backslash G)$ into irreducible unitary representations of $G$ it is enough to show that if $\pi$ is an irreducible unitary representation for which

$$
\operatorname{Hom}_{K}\left(\wedge^{*} \mathfrak{p} \otimes F, \pi\right) \neq 0,
$$

there exists a positive constant $\varepsilon$ depending only on $F$ such that ${ }^{8}$

$$
\Lambda_{F}-\Lambda_{\pi} \geqslant \varepsilon
$$

To do so we proceed as in the proof of [10, §II, Proposition 6.12].

Fix compatible (in the sense of $[10, \mathrm{II}, \S 6.6]$ ) positive root systems $\Delta^{+}$and $\Delta_{k}^{+}$for $\mathfrak{g} \otimes \mathbb{C}$ and $\mathfrak{k} \otimes \mathbb{C}$ and denote by $\rho$ and $\rho_{k}$ the corresponding half-sums of positive roots. Let $W$ be the Weyl group of $\mathfrak{g}_{\mathbb{C}}$ and set $W^{1}=\left\{w \in W: w \Delta^{+}\right.$is compatible with $\left.\Delta_{k}^{+}\right\}$. Write $v$ for the highest weight of $F$; note that $\Lambda_{F}=|v+\rho|^{2}-|\rho|^{2}$.

The orthogonal Lie algebra $\mathfrak{s o}(\mathfrak{p})$ has a natural representation $S=\operatorname{spin}(\mathfrak{p})$. Since the adjoint action of $\mathfrak{k}$ on $\mathfrak{p}$ preserves the Killing form defining $\mathfrak{s o}(\mathfrak{p})$, there is a natural map $\mathfrak{k} \rightarrow \mathfrak{s o}(\mathfrak{p})$; so $S$ may be regarded as a representation of $\mathfrak{k}$. The exterior algebra $\wedge^{*} \mathfrak{p}$ is isomorphic to one or two copies (according to the parity of the dimension of $\mathfrak{p}$ ) of $S \otimes S$. Since $S$ is isomorphic to $S^{*}$ as a $\mathfrak{k}$-module, it follows from (4.1.1) that there exists a representation of $\mathfrak{k}$ occurring in both $F \otimes S$ and $\pi \otimes S$. Now [10, §II, Lemma 6.9] implies that every highest weight of $F \otimes S$ is of the form $\frac{1}{2}(\mu+\theta \mu)+w \rho-\rho_{k}$ where $\mu$ is a weight of $F$ and $w \in W^{1}$. Using the expression relating the square of the Dirac operator $D$ to the Casimir operator (see [10, §II, Lemma 6.11]), and the positivity of $D$, we conclude that there exists a weight $\mu$ of $F$ and an element $w$ of $W^{1}$ such that

$$
\left|\frac{1}{2}(\mu+\theta \mu)+w \rho\right|^{2} \geqslant \Lambda_{\pi}+|\rho|^{2} .
$$

This inequality is a refined version of Parthasarathy's Dirac operator inequality $[58,(2.26)]$. It implies that

$$
\Lambda_{F}-\Lambda_{\pi} \geqslant|v+\rho|^{2}-\left|\frac{1}{2}(\mu+\theta \mu)+w \rho\right|^{2} .
$$

Now since $\left|\frac{1}{2}(\mu+\theta \mu)+w \rho\right|^{2} \leqslant|\mu+w \rho|^{2}$, the left-hand side of (4.1.2) is nonnegative: in fact $\mu+w \rho$ is a weight of the finite dimensional representation of $\mathfrak{g}$ given by $F \otimes F_{\rho}$,

${ }^{8}$ Recall from (3.6.1) that if the space of $\pi$ is realized as a subspace of $L^{2}(\Gamma \backslash G)$ the Laplace operator acts on the corresponding subspace of $\operatorname{Hom}_{K}\left(\wedge^{*} \mathfrak{p} \otimes F, L^{2}(\Gamma \backslash G)\right)$ through $\Lambda_{F}-\Lambda_{\pi}$. 
where $F_{\rho}$ has highest weight $\rho$; thus

$$
|\mu+w \rho| \leqslant|v+\rho|
$$

with equality if and only if there exists $u \in W$ with $\mu+w \rho=u(v+\rho)$. But since $w \rho$ is $\theta$-invariant we conclude that the left-hand side of (4.1.2) vanishes if and only if

$$
|v+\rho|^{2}=\left|\frac{1}{2}(u(v+\rho)+\theta u(v+\rho))\right|^{2} .
$$

This forces $u(v+\rho)$ to be $\theta$-fixed, i.e., the isomorphism class of $F$ to be $\theta$-fixed.

From now on we assume that $\rho$ is strongly acyclic.

We denote by $T_{X}(\rho)$ the analytic torsion of $\left(X, E_{\rho}\right)$ w.r.t. the metric on $E_{\rho}$ that we fixed in $\S 3.4$; it is defined as (cf. §2)

$$
\log T_{X}(\rho)=\frac{1}{2} \sum_{k \geqslant 0}(-1)^{k+1} k \log \operatorname{det}^{\prime} \Delta_{k} .
$$

We recall the definition of $\operatorname{det}^{\prime} \Delta_{k}$ in $\S 4.2$ below. It follows from (2.3.4) and (2.3.3) that, if $\rho$ is strongly acyclic and we are given a local system $M$ of free $\mathbb{Z}$-modules with an isomorphism $M \otimes \mathbb{C} \stackrel{\sim}{\longrightarrow} E_{\rho}$, we have

$$
\log T_{X}(\rho)=-\sum^{*} \log \left|H^{i}(X ; M)\right| .
$$

\section{2 .}

Although already discussed - somewhat informally - in $\S 2$, let us recall the definition of $\operatorname{det}^{\prime} \Delta_{k}$.

The Laplacian $\Delta_{k}$ is a symmetric, positive definite, elliptic operator with pure point spectrum

$$
0<\lambda_{1} \leqslant \lambda_{2} \leqslant \cdots \rightarrow+\infty
$$

and, writing $\mathrm{e}^{-t \Delta_{k}}(x, y)(x, y \in X)$ for the integral kernel representing the heat kernel on $k$-forms on $X$,

$$
\operatorname{Tre}^{-t \Delta_{k}}=\int_{X} \operatorname{tr}\left(\mathrm{e}^{-t \Delta_{k}}(x, x)\right) d x=\sum_{j=1}^{+\infty} \mathrm{e}^{-t \lambda_{j}}
$$

is convergent for each positive $t$. We may thus define

$$
\log \operatorname{det}^{\prime} \Delta_{k}=-\frac{d}{d s} \zeta_{k}(s ; \rho)_{\mid s=0}
$$

where the function $\zeta_{k}$ is the unique meromorphic function [62] satisfying

$$
\zeta_{k}(s ; \rho)=\frac{1}{\Gamma(s)} \int_{0}^{+\infty} t^{s-1} \operatorname{Tre}^{-t \Delta_{k}} d t=\sum_{j=1}^{+\infty} \lambda_{j}^{-s} .
$$

for $\operatorname{Re}(s)$ sufficiently large. 


\section{3. $\Delta_{k}$ and $\Delta_{k}^{(2)}$}

Our goal is to relate the analytic torsion on $X$ to computations on the universal covering $S$; and in particular, as the injectivity radius of $X$ tends to infinity, to approximate the former by the latter.

Indeed, if we write $\mathrm{e}^{-t \Delta_{k}}(x, y)(x, y \in X)$ for the integral kernel representing the heat kernel on $k$-forms on $X$, then for each positive $t$ and for each integer $k$ we have

$$
\mathrm{e}^{-t \Delta_{k}}(x, y)=\sum_{\gamma \in \Gamma}\left(\gamma_{y}\right)^{*} \mathrm{e}^{-t \Delta_{k}^{(2)}}(\tilde{x}, \gamma \tilde{y})
$$

where $\tilde{x}, \tilde{y}$ are lifts of $x, y$ to $S$; by $\left(\gamma_{y}\right)^{*}$, we mean the pullback by the map $(x, y) \mapsto(x, \gamma y)$. The sum converges absolutely and uniformly for $\tilde{x}, \tilde{y}$ in compacta: by cocompactness of $\Gamma$ and bounds on volume growth, there exist constants $c_{i}=c_{i}(\Gamma, G)$, $i=2,3$, such that for any $x, y \in S$,

$$
N(x, y ; R):=|\{\gamma \in \Gamma: d(\tilde{x}, \gamma \tilde{y}) \leqslant R\}| \leqslant c_{2} e^{c_{3} R}
$$

(see e.g. [40]) which taken in combination with Lemma 3.8 gives the absolute convergence. In particular, when the injectivity radius is big, a single term in (4.3.1) will dominate.

\section{4. $L^{2}$-torsion}

The trace of the $\left(S\right.$-)heat kernel $\mathrm{e}^{-t \Delta_{k}^{(2)}}(x, x)$ on the diagonal is independent of $x$, because it is invariant under $G$. We will prove in $\S 5$ that the integral

$$
\frac{1}{\Gamma(s)} \int_{0}^{+\infty} t^{s-1} \operatorname{tr} \mathrm{e}^{-t \Delta_{k}^{(2)}}(x, x) d t
$$

is absolutely convergent for $\operatorname{Re}(s)$ sufficiently large and extends to a meromorphic function of $s \in \mathbb{C}$ which is holomorphic at $s=0$.

Define $t_{S}^{(2)}(\rho)$ via

$$
t_{S}^{(2)}(\rho)=\frac{1}{2} \sum_{k \geqslant 0}(-1)^{k} k\left(\left.\frac{d}{d s}\right|_{s=0} \frac{1}{\Gamma(s)} \int_{0}^{+\infty} t^{s-1} \operatorname{tr} \mathrm{e}^{-t \Delta_{k}^{(2)}}(x, x) d t\right) .
$$

(The product of $t_{S}^{(2)}(\rho)$ with the volume of $X$ is the $L^{2}$-analytic torsion of $X$.)

Remark. The $L^{2}$-analytic torsion is generally defined as

$$
\begin{aligned}
t_{S}^{(2)}(\rho)= & \frac{1}{2} \sum_{k \geqslant 0}(-1)^{k} k\left(\left.\frac{d}{d s}\right|_{s=0} \frac{1}{\Gamma(s)} \int_{0}^{\varepsilon} t^{s-1} \operatorname{tr} \mathrm{e}^{-t \Delta_{k}^{(2)}}(x, x) d t\right) \\
& +\frac{1}{2} \sum_{k \geqslant 0}(-1)^{k} k \int_{\varepsilon}^{+\infty} t^{-1} \operatorname{tr} \mathrm{e}^{-t \Delta_{k}^{(2)}}(x, x) d t
\end{aligned}
$$

under the condition that for some positive $\varepsilon$ each integral

$$
\int_{\varepsilon}^{+\infty} t^{-1} \operatorname{tr} \mathrm{e}^{-t \Delta_{k}^{(2)}}(x, x) d t
$$


is convergent. For trivial coefficients this last condition is well known to be satisfied: symmetric spaces are of 'analytic determinant class' is the sense of [49]. The condition is still satisfied for general $\rho$, as will follow from the proof of Proposition 5.2.

It is possible to compute $t_{S}^{(2)}(\rho)$ in a completely explicit fashion - it is an explicit quantity depending on $\rho$ - and we carry this out in $\S 5$. Thus the following theorem shows that we can approximate the torsion of any 'large' quotient $X$ by an explicitly computable quantity.

Theorem 4.5. Let $\rho: G \rightarrow \mathrm{GL}(E)$ be a strongly acyclic representation. Then,

$$
\frac{\log \left(T_{X}(\rho)\right)}{\operatorname{vol}(X)} \rightarrow t_{S}^{(2)}(\rho)
$$

when $\Gamma$ varies through any sequence of subgroups for which the injectivity radius of $X=\Gamma \backslash S$ goes to $\infty$.

We conjecture that this remains valid without the strongly acyclic assumption. We are unable to make significant progress without ruling out eigenvalues on $k$-forms very close to zero. At a combinatorial level this seems to be equivalent to exceptionally difficult problems of diophantine approximation.

The scheme of proof will be, when unwound, very close to the latter part of the discussion in $\S 2.5$. In the language of $\S 2.5$, we already observed in that context that difficulties arose when $\operatorname{Im}\left(\lambda_{j}\right)=0$, which manifested themselves also arithmetically through (2.5.3). This is the issue of 'small eigenvalues'.

Proof. Let $\ell$ be the length of the shortest closed geodesic on $X$. Assume for simplicity that $\ell \geqslant 1$. Let

$$
A_{k}(t)=\frac{1}{\operatorname{vol}(X)} \int_{X}\left(\operatorname{tr} \mathrm{e}^{-t \Delta_{k}^{(2)}}(\tilde{x}, \tilde{x})-\operatorname{tr} \mathrm{e}^{-t \Delta_{k}}(x, x)\right) d x,
$$

where $\tilde{x}$ denotes any lift of $x$ to $S$ (in fact, $\operatorname{tr} \mathrm{e}^{-t \Delta_{k}^{(2)}}$ is constant, and so we could replace $\tilde{x}$ by a fixed basepoint in what follows).

Remark. Both $\int_{1}^{+\infty} \int_{X} \operatorname{tr} \mathrm{e}^{-t \Delta_{k}^{(2)}}(\tilde{x}, \tilde{x}) \frac{d t}{t}$ and $\int_{1}^{+\infty} \int_{X} \operatorname{tr} \mathrm{e}^{-t \Delta_{k}}(x, x) \frac{d t}{t}$ are known to be convergent. In our case, where the local system is strongly acyclic we will easily prove below that both of these integrals are even absolutely convergent.

The following lemma is a slight modification of [46, Lemma 4]:

Lemma. Let $M$ be a real number $\geqslant 1$. There exists a constant $c=c(M, \rho, G)$ such that for any $t \in(0, M]$,

$$
\left|A_{k}(t)\right| \leqslant c t^{-(d+1) / 2} e^{-\frac{(\ell-c t)^{2}}{5 t}} .
$$

Proof. We may rewrite (4.3.1) as

$$
\mathrm{e}^{-t \Delta_{k}}(x, x)-\mathrm{e}^{-t \Delta_{k}^{(2)}}(\tilde{x}, \tilde{x})=\sum_{\gamma \in \Gamma, \gamma \neq e}\left(\gamma_{y}\right)^{*} \mathrm{e}^{-t \Delta_{k}^{(2)}}(\tilde{x}, \gamma \tilde{x})
$$


It follows from Lemma 3.8 and (4.3.2) that, up to constants, the last sum is bounded by

$$
\int_{\ell}^{+\infty} t^{-d / 2} e^{-r^{2} / 5 t} d(N(x, x ; r)-N(x, x ; \ell)) \leqslant c_{2} \int_{\ell}^{+\infty}\left|\frac{d}{d r}\left\{t^{-d / 2} e^{-r^{2} / 5 t}\right\}\right| e^{c_{3} r} d r .
$$

And the last integral above is, up to a constant, bounded by

$$
\begin{aligned}
t^{-(d+1) / 2} \int_{\ell}^{+\infty} e^{-r^{2} / 5 t} r e^{c_{3} r} d r & \leqslant \text { const } \cdot t^{-(d+1) / 2} e^{-\left(\ell-3 c_{3} t\right)^{2} / 5 t} \int_{\ell}^{+\infty} r e^{-c_{3} r / 5} d r \\
& \leqslant \text { const } \cdot t^{-(d+1) / 2} e^{-\left(\ell-3 c_{3} t\right)^{2} / 5 t}
\end{aligned}
$$

Integrating (4.5.2) over $X$ thus gives the lemma.

It follows from (4.5.1) that

$$
\int_{0}^{1} t^{s-1} A_{k}(t) d t
$$

is holomorphic in $s$ in a half-plane containing 0 , so (using the remark above)

$$
\left.\frac{d}{d s}\right|_{s=0} \frac{1}{\Gamma(s)} \int_{0}^{+\infty} t^{s-1} A_{k}(t) d t=\int_{0}^{+\infty} A_{k}(t) \frac{d t}{t} .
$$

It thus follows from the definitions that

$$
t_{S}^{(2)}(\rho)-\frac{\log \left(T_{X}(\rho)\right)}{\operatorname{vol}(X)}=\frac{1}{2} \sum_{k=0}^{d}(-1)^{k} k \int_{0}^{+\infty} A_{k}(t) \frac{d t}{t} .
$$

It is to handle the 'large $t$ ' contribution to $\int_{0}^{\infty} A_{k}(t) \frac{d t}{t}$ that 'strong acyclicity' of $\rho$ enters. Let $\eta$ be such that every eigenvalue of $\Delta_{k}$ is $\geqslant \eta$. Now, for any $t \geqslant 1$, spectral expansion on the compact manifold $\Gamma \backslash S$ shows that

$$
\operatorname{tr} \mathrm{e}^{-t \Delta_{k}}(x, x) \leqslant e^{-\eta(t-1)} \operatorname{tr} e^{-\Delta_{k}}(x, x) .
$$

We apply (4.3.1) and Lemma 3.8 with $M$ replaced by 1 , say, to estimate the latter quantity, arriving at

$$
\operatorname{tr} \mathrm{e}^{-t \Delta_{k}(x, x)} \leqslant c^{\prime} e^{-\eta(t-1)},
$$

where the constant $c^{\prime}$ only depends on $\rho$ and $G$. A similar reasoning with $\mathrm{e}^{-t \Delta_{k}^{(2)}}(\tilde{x}, \tilde{x})$ leads to the conclusion that both

$$
\int_{1}^{\infty} t^{-1} \operatorname{tr} \mathrm{e}^{-t \Delta_{k}^{(2)}}(\tilde{x}, \tilde{x}) d t, \quad \int_{1}^{\infty} t^{-1} \operatorname{tr} \mathrm{e}^{-t \Delta_{k}}(x, x) d t
$$

are absolutely convergent. Thus, given $v>0$ arbitrary, there exists a constant $M \geqslant 1$ depending only on $G, \rho$ and $v$ such that

$$
\left|\int_{M}^{+\infty} t^{-1} A_{k}(t) d t\right| \leqslant v .
$$

But it follows from (4.5.1) that

$$
\left|\int_{0}^{M} t^{-1} A_{k}(t) d t\right| \rightarrow 0
$$


as the injectivity radius $\ell$ tends to $\infty$. In particular, this shows that the right-hand side of (4.5.2) approaches zero as the injectivity radius $\ell$ approaches $\infty$.

\section{Explicit evaluation of the $\boldsymbol{L}^{2}$-torsion for unimodular local systems}

In this section, we shall compute explicitly the asymptotic constants of Theorem 4.5. The contents of this section are extensions of computations from [57] and also relate to computations performed in [20].

\subsection{Notation}

We continue with notation as in $\S 3$ with the following change: in what follows, if $H$ is a real Lie group, we will write $\mathfrak{h}$ for the complexification of its real Lie algebra, and $\mathfrak{h}_{\mathbb{R}}$ for the Lie algebra of $H$.

5.1.1. Groups and subgroups. We have already defined $K \subset G$ and $U$ a compact form of $G$. Note that we may identify the complexified Lie algebra $\mathfrak{u}$ of $U$ and the complexified Lie algebra of $\mathfrak{g}$. Let $S=G / K$ be the Riemannian symmetric space associated with $G$ and $S^{c}=U / K$ be its compact dual. Let $\Theta$ be the Cartan involution of $G$ fixing $K$.

Fix a maximal torus $T_{f} \subset K$ with complexified Lie algebra $\mathfrak{b}$. Extend it to a $\Theta$-stable maximal torus $\mathfrak{t}_{U}=\mathfrak{b} \oplus \mathfrak{a}_{0} \subset \mathfrak{u}$, where $\mathfrak{a}_{0} \subset \mathfrak{p}$ is the complexification of an abelian subspace $\mathfrak{a}_{0 \mathbb{R}} \subset \mathfrak{p}_{\mathbb{R}}$. Therefore, $\mathfrak{b} \oplus \mathfrak{a}_{0}$ is a 'fundamental Cartan subalgebra', i.e. one with maximal compact part. We extend $\mathfrak{a}_{0 \mathbb{R}}$ to an 'Iwasawa' space $\mathfrak{a}_{\mathbb{R}} \subset \mathfrak{p}_{\mathbb{R}}$ - that is to say, a maximal abelian semisimple subspace.

Let $T_{U} \subset U, W_{U} \subset \operatorname{Aut}\left(\mathfrak{t}_{U}\right)$ be the maximal tori and Weyl groups that correspond to $\mathfrak{t}_{U} \subset \mathfrak{g}$.

Let $A_{f}=\exp \left(\mathfrak{a}_{0 \mathbb{R}}\right)$. Then $A_{f}$ is the split part of a fundamental parabolic subgroup $P_{f}=M_{f} A_{f} N_{f}$; here $M_{f} A_{f}$ is the centralizer in $G$ of $\mathfrak{a}_{0}$, and the $\Theta$-stable subgroup $M_{f}$ is a certain canonical complement to $A_{f}$ (see [39, Chapter V, $\left.\S 5\right]$ ).

Let $K_{f}=K \cap M_{f}$; it is a maximal compact subgroup of $M_{f}$. We denote by $M_{f}^{0}$ and $K_{f}^{0}$ the connected components of $M_{f}$ and $K_{f}$ respectively. Denote by $\mathfrak{k}_{f}$ and $\mathfrak{m}_{f}$ the complexified Lie algebras of $K_{f}$ and $M_{f}$. Note that $T_{f} \subset M_{f}$ and $T_{f} \subset K_{f}^{0}$.

We let $W_{f}$ be the Weyl group for $\mathfrak{b} \subset \mathfrak{k}_{f}$ and $W_{M_{f}}$ be the Weyl group of $\mathfrak{b} \subset \mathfrak{m}_{f}$. Note that, in general, the natural injection $W_{f} \hookrightarrow W_{M_{f}}$ may not be surjective.

We say, as usual, that a parabolic subgroup is standard if it contains $A=\exp \left(\mathfrak{a}_{0 \mathbb{R}}\right)$. Finally let $Q$ be another standard parabolic subgroup with Levi decomposition ${ }^{9}$ $Q=M_{Q} A_{Q} N_{Q}$.

5.1.2. Bilinear forms. We equip $\mathfrak{t}_{U}$ with the bilinear form which is the negative of the Killing form for $U$; we equip $\mathfrak{a}_{0}$ and $\mathfrak{a}$ with the bilinear form defined by the Killing form for $G$. These forms are all nondegenerate, and thereby equip the dual spaces $\mathfrak{t}_{U}^{*}, \mathfrak{a}_{0}^{*}, \mathfrak{a}^{*}$ with bilinear forms; if we write, for instance, $\langle\alpha, \beta\rangle$ for some $\alpha, \beta \in \mathfrak{a}^{*}$, it always means that this is taken with respect to the bilinear form just normalized.

${ }^{9}$ Note that neither $M_{Q}$ nor $M_{f}$ need be connected. 
5.1.3. Systems of positive roots. We choose a system $\Delta^{+}\left(\mathfrak{b}, \mathfrak{m}_{f}\right)$ of positive roots for the action of $\mathfrak{b}$ on $\mathfrak{m}_{f}$; it restricts to a system of positive roots for $\mathfrak{b}$ on $\mathfrak{k}_{f}$. We extend it to a system $\Delta^{+}=\Delta^{+}\left(\mathfrak{t}_{U}, \mathfrak{u}\right)$ of positive roots for the action of $\mathfrak{t}_{U}$ on $\mathfrak{u}$ : a $\operatorname{root} \alpha$ of $\mathfrak{t}_{U}$ on $\mathfrak{u}$ is positive if and only if either the corresponding root space belongs to $\mathfrak{n}_{f}$, the complexified Lie algebra of $N_{f}$; or, otherwise, $\alpha$ is trivial on $\mathfrak{a}_{0}$ and $\alpha \in \Delta^{+}\left(\mathfrak{b}, \mathfrak{m}_{f}\right)$.

Let $\rho_{U} \in \mathfrak{t}_{U}^{*}$ be the half-sum of positive roots in $\Delta^{+}$. Define similarly $\rho_{M_{f}}, \rho_{K_{f}}$.

5.1.4. Representations. Let $\rho_{\lambda}$ be an irreducible representation of $U$ with (dominant) highest weight $\lambda \in \mathfrak{t}_{U}^{*}$; it extends to a unique holomorphic representation of $G_{\mathbb{C}}$, which we also denote as $\rho_{\lambda}$.

Given $\theta$, a unitary representation of $M_{Q}$ and $v \in \mathfrak{a}_{Q}^{*}=\operatorname{Hom}\left(\mathfrak{a}_{Q}, \mathbb{C}\right)$ we may construct a (generalized) principal series representation $\pi(\theta, v)$ of the group $G$; its restriction to $K$ is isomorphic to $\operatorname{Ind}_{\left(K \cap M_{Q}\right)}^{K} \theta$. We normalize so that $\pi(\theta, v)$ is tempered unitary if $\theta$ is tempered and $\nu \mid \mathfrak{a}_{Q, \mathbb{R}}$ is valued in $i \mathbb{R}$. Note that this principal series is not necessarily irreducible; however, for $\theta$ irreducible and generic $v$, it is indeed irreducible [39, Theorem 7.2].

For each $w \in W_{U}$, let $\mu_{w}$ be the restriction of $w\left(\rho_{U}+\lambda\right)$ to $\mathfrak{b}$. For any $v \in \mathfrak{a}^{*}$, we regard $\mu_{w}+v$ as defining an element of $(\mathfrak{a} \oplus \mathfrak{b})^{*}=\mathfrak{t}_{U}^{*}$, in the obvious way. Put

$$
P_{w}(v)=\operatorname{sign}(w) \prod_{\alpha \in \Delta^{+}\left(\mathfrak{t}_{U}, \mathfrak{u}\right)} \frac{\left\langle\mu_{w}+v, \alpha\right\rangle}{\left\langle\rho_{U}, \alpha\right\rangle},
$$

where $\langle-,-\rangle$ is induced by the Killing form, as described above. Then $P_{w}$ depends only on the coset of $w$ in $W_{M_{f}} \backslash W_{U}$. Indeed, for $w^{\prime} \in W_{M_{f}}, \prod_{\alpha}\left\langle\mu_{w^{\prime} w}+v, \alpha\right\rangle=\prod_{\alpha}\left\langle\mu_{w}+v, w^{\prime-1} \alpha\right\rangle$ and the set $w^{\prime-1} \alpha$ coincides with the set of $\alpha$ after a number of sign changes, of total parity $\operatorname{sign}\left(w^{\prime}\right)$.

Proposition 5.2. If $\delta(S) \neq 1$, then $t_{S}^{(2)}(\rho)=0$. If $\delta(S)=1$, for each $w \in W_{U}$, put $J_{w}=\left\{v \in \operatorname{Hom}\left(\mathfrak{a}_{0, \mathbb{R}}, \mathbb{R}\right):|v| \leqslant \sqrt{\left|\rho_{U}+\lambda\right|^{2}-\left|\mu_{w}\right|^{2}}\right\} ;$ equip $\operatorname{Hom}\left(\mathfrak{a}_{0, \mathbb{R}}, \mathbb{R}\right)$ with the additive-invariant measure in which the interval $|\nu| \leqslant 1$ has measure 2 . Then there exists $c(S)>0$, depending only on $S$, such that

$$
t_{S}^{(2)}(\rho)=(-1)^{\frac{\operatorname{dim} S-1}{2}} c(S) \sum_{w \in W_{M_{f}} \backslash W_{U}} \int_{J_{w}} P_{w}(\nu) d \nu .
$$

Moreover, when $\delta(S)=1$,

$$
(-1)^{\frac{\operatorname{dim} S-1}{2}} t_{S}^{(2)}(\rho)>0
$$

Note that $t_{S}^{(2)}(\rho)$ depends on the choice of a normalization for the (symmetric) Riemannian metric on $S$. If we had scaled the metric on $S$ by a constant $C$, then $t_{S}^{(2)}(\rho)$ would have been scaled by $C^{-\operatorname{dim}(S)}$, as is the volume form on $S$.

The proof takes up the rest of this section; we work out some explicit examples in $\S 5.9$ and prove that $t_{S}^{(2)}(\rho)$ is nonzero if $\delta(S)=1$ in $\S 5.8$. Let us explain the outline. We are not going to give a lot of details of issues related to convergence here. It follows 
from $\S 3.6$ and (4.4.1) (see also $[57,(12)]$ for more details) that

$$
t_{S}^{(2)}(\rho)=\left.\frac{1}{2} \frac{d}{d s}\right|_{s=0} \frac{1}{\Gamma(s)} \int_{0}^{\infty} d t \int_{\pi \in \hat{G}} d(\pi) t^{s-1} \mathrm{e}^{-t\left(\Lambda_{\rho}-\Lambda_{\pi}\right)} d \mu_{\operatorname{Planch}}(\pi)
$$

where $d(\pi) \in \mathbb{Z}$ is defined as

$$
d(\pi):=\sum_{k}(-1)^{k} k \operatorname{dim}\left[\left.\pi \otimes \wedge^{k} \mathfrak{p}^{*} \otimes \rho_{\lambda}\right|_{K}\right]^{K},
$$

$d \mu_{\text {Planch }}$ is the Plancherel measure on the unitary dual $\hat{G}$, and $\Lambda_{\rho}, \Lambda_{\pi}$ denote the Casimir eigenvalues of $\rho$ and $\pi$. The convergence of the right-hand side follows from $\S 5.7$. It is often convenient to think of $d(\pi)$ as the dimension of the virtual vector space $\sum^{*} k\left[\left.\pi \otimes \wedge^{k} \mathfrak{p}^{*} \otimes \rho_{\lambda}\right|_{K}\right]^{K}$ (the alternating sum). Note that $\wedge^{k_{\mathfrak{p}}}$ and $\wedge^{\operatorname{dim} S-k_{\mathfrak{p}} \text { are }}$ isomorphic $K$-modules. The representation $\left.\rho_{\lambda}\right|_{K}$ being self-dual, it therefore follows that $d(\pi)=0$ if $S$ is even dimensional (see the proof [55, Proposition 2.9] for more details), so $t_{S}^{(2)}(\rho)=0$ as long as $S$ is even dimensional.

The proof of Proposition 5.2 follows from the following results:

(1) $d(\pi(\theta, \nu))=0$ unless $Q$ is associated with $P_{f}$. We prove this in $\S 5.3$. This proves, by standard facts about the Plancherel measure, that the integration in (5.2.1) may be restricted to the case where $Q=P_{f}, \operatorname{dim} A_{f}=1$ and $\theta$ is a discrete-series representation of $M=M_{f}$.

(2) In $\S \S 5.4-5.6$, we evaluate $d(\pi(\theta, v))$ explicitly when $Q=P_{f}$ is a fundamental parabolic subgroup and $\theta$ a discrete series; namely, $\S 5.4$ sets up general notation, $\S 5.5$ recalls character formulas, and $\S 5.6$ carries out the computation of $d(\pi)$.

(3) The explicit Plancherel density when $Q=P_{f}$ and $\theta$ is discrete is presented in (5.4.2); this, together with $\S 2.8$, completes the computation of the right-hand side of (5.2.1).

\section{3.}

Let $G$ be a group and $V$ a $G$-vector space. We denote by $\operatorname{det}[1-V]$ the virtual $G$-representation (that is to say, the element of $K_{0}$ of the category of $G$-representations) defined by the alternating sum $\sum_{i}(-1)^{i}\left[\wedge^{i} V\right]$ of exterior powers. This is multiplicative in an evident sense:

$$
\operatorname{det}[1-V \oplus W]=\operatorname{det}[1-V] \otimes \operatorname{det}[1-W] .
$$

We put $\operatorname{det}^{\prime}[1-V]=\sum_{i}(-1)^{i} i \wedge^{i} V$. Then

$$
\operatorname{det}^{\prime}[1-V \oplus W]=\operatorname{det}^{\prime}[1-V] \otimes \operatorname{det}[1-W] \oplus \operatorname{det}[1-V] \otimes \operatorname{det}^{\prime}[1-W] .
$$

Let us note that if $H$ is a group acting on $V$ such that every $g \in H$ has a fixed space of dimension $\geqslant 2$, then $\operatorname{det}^{\prime}[1-V]$ is trivial as a virtual $H$-representation; this follows since, by (5.3.2), the trace of every $g \in H$ on $\operatorname{det}^{\prime}[1-V]$ is then trivial.

Note that $\operatorname{det}^{\prime}\left[1-\mathfrak{p}^{*}\right]=\operatorname{det}^{\prime}[1-\mathfrak{p}]$ as virtual $K$-representations so, by Frobenius reciprocity and (5.2.2),

$$
d(\pi(\theta, v))=\operatorname{dim}\left[\theta \otimes \operatorname{det}^{\prime}[1-\mathfrak{p}] \otimes \rho_{\lambda}\right]^{\left(K \cap M_{Q}\right)} .
$$


Let us suppose that (5.3.3) is nonzero. Each $g \in K \cap M_{Q}$ fixes, in its conjugation action, $\mathfrak{a}_{Q}$; on the other hand, it also belongs to some conjugate of a maximal torus in $K$ and, hence, fixes some $K$-conjugate of $\mathfrak{a}_{0}$. There must exist some $g \in K \cap M_{Q}$ for which these two spaces coincide and are one dimensional; otherwise $\operatorname{det}^{\prime}\left[1-\mathfrak{p}^{*}\right]$ is virtually trivial. Therefore, $\mathfrak{a}_{0}$ and $\mathfrak{a}_{Q}$ are $K$-conjugate and of dimension at most 1 . The case where $\delta(S)=0$ is excluded, as this would force $\operatorname{dim} S$ to be even and thus (5.3.3) to be zero. It therefore follows that $Q$ is fundamental (i.e., it is associated with $P_{f}$ ) and $\operatorname{dim} A_{f}=1$.

In particular, $t_{S}^{(2)}(\rho)=0$ unless $\delta(S)=1$.

\subsection{The case of the fundamental series.}

In the remainder of the section, $Q=P_{f}$.

It is a theorem of Harish-Chandra [39, Corollary 14.60] that, in these circumstances, $\pi(\theta, v)$ is irreducible for all $v$, although we do not need this fact. Since compact Cartan subgroups of $M_{f}$ are connected, we may moreover realize $\theta$ as an induction from the connected component $M_{f}^{0}$ of a discrete-series representation $\theta_{0}$ of $M_{f}^{0}$ (see $[69, \S \S 6.9$ and 8.7.1]). Again by Frobenius reciprocity we obtain

$$
d(\pi(\theta, v))=\operatorname{dim}\left[\theta_{0} \otimes \operatorname{det}^{\prime}[1-\mathfrak{p}] \otimes \rho_{\lambda}\right]^{K_{f}^{0}} .
$$

We denote by $\mu \in \mathfrak{b}^{*}$ the infinitesimal character of the representation $\theta_{0}^{*}$ - the representation dual to $\theta$. (We index $\theta$ by the infinitesimal character of its dual to make the computation shorter at a later stage.) Therefore, $\theta_{0}^{*}$ contains with multiplicity 1 the $K_{f}^{0}$ type with highest weight $\mu+\rho_{M_{f}}-2 \rho_{K_{f}}$ ('Blattner's formula', [39, Theorem 9.20]). There exists a positive constant $c_{S}^{\prime}$ depending only on our choice of a Haar measure on $G$ such that the Plancherel measure along the space of $\pi(\theta, v)$ is equal to

$$
c_{S}^{\prime}(-1)^{\frac{1}{2} \operatorname{dim} N} \prod_{\alpha \in \Delta^{+}} \frac{\langle\mu+v, \alpha\rangle}{\left\langle\rho_{U}, \alpha\right\rangle} \cdot d v
$$

(see [39, Theorem 13.11]). This is a nonnegative even polynomial on $i \mathfrak{a}_{0, \mathbb{R}}$.

\subsection{Weyl denominators}

We continue with the notation of $\S 5.4$. Let $T_{f}^{\sim}, T_{U}^{\sim}$ denote, respectively, the universal covers of $T_{f}, T_{U}$.

Our choice of positive systems defines Weyl denominators $D_{M_{f}}: T_{f}^{\sim} \rightarrow \mathbb{C}, D_{U}: T_{U}^{\sim} \rightarrow$ $\mathbb{C}$; these are, by definition, the determinant of $1-\operatorname{Ad}\left(t^{-1}\right)$ acting on the sums $\mathfrak{m}_{f}^{+}$and $\mathfrak{u}^{+}$respectively of all positive root spaces on $\mathfrak{m}_{f}$ and $\mathfrak{u}$, respectively, multiplied by $\rho_{M_{f}}$ or $\rho_{U}$. In a similar way we obtain a Weyl denominator $D_{K_{f}}$ of $T_{f}^{\sim}$, where we sum over all positive roots of $\mathfrak{k}_{f}$ only. Note that formally Weyl denominators are given by products $\prod_{\alpha>0}\left(e^{\alpha / 2}-e^{-\alpha / 2}\right)$ over the corresponding positive roots.

Now $\mathfrak{u} / \mathfrak{t}_{U}=\mathfrak{u}^{+} \oplus \mathfrak{u}^{-}$and (5.3.1) implies that the character of $\operatorname{det}\left[1-\mathfrak{u} / \mathfrak{t}_{U}\right]$ is equal to $\prod_{\alpha>0}\left(1-e^{\alpha}\right) \cdot \prod_{\alpha>0}\left(1-e^{-\alpha}\right)$. It follows that

$$
D_{U}^{2}=(-1)^{\operatorname{dim} \mathfrak{u}^{+}} \text {character of } \operatorname{det}\left[1-\mathfrak{u} / \mathfrak{t}_{U}\right],
$$

with a similar identity for $D_{M_{f}}^{2}$ and $D_{K_{f}}^{2}$. 
We claim that, as characters of $T_{f}^{\sim}$,

$$
\left|D_{K_{f}}\right|^{2} \cdot\left(\text { character of } \operatorname{det}^{\prime}[1-\mathfrak{p}]\right)=-D_{U} \overline{D_{M_{f}}}
$$

(and in particular, the right-hand side descends to $T_{f}$ ).

Equivalently, since the $T_{f}$-character of $\operatorname{det}^{\prime}[1-\mathfrak{p}]$ is the negative of the character of $\operatorname{det}\left[1-\mathfrak{p} / \mathfrak{a}_{0}\right]$ and $D_{K_{f}}^{2}$ (resp. $\left.D_{M_{f}}^{2}\right)$ is real valued and has the same sign as $(-1)^{\operatorname{dim} \mathfrak{k}_{f}^{+}}$ (resp. $(-1)^{\operatorname{dim} \mathfrak{m}_{f}^{+}}$), we need to establish

$$
\operatorname{det}\left[1-\mathfrak{p} / \mathfrak{a}_{0}\right]=(-1)^{\operatorname{dim} \mathfrak{m}_{f}^{+}+\operatorname{dim} \mathfrak{k}_{f}^{+}} \frac{D_{U}}{D_{M_{f}}} \cdot \frac{D_{M_{f}}^{2}}{D_{K_{f}}^{2}} .
$$

The signs of both sides of this putative equality are the same. In fact, we claim that $(-1)^{\operatorname{dim} \mathfrak{m}_{f}^{+}+\operatorname{dim} \mathfrak{k}_{f}^{+}} D_{M_{f}}^{2} / D_{K_{f}}^{2}, D_{U} / D_{M_{f}}$, and the character of $\operatorname{det}\left[1-\mathfrak{p} / \mathfrak{a}_{0}\right]$ all take positive values on $T_{f}^{\sim}$ :

- $D_{M_{f}}^{2}$ is real valued and has the same sign as $(-1)^{\operatorname{dim} \mathfrak{m}_{f}^{+}} ;$and similarly for $D_{K_{f}}^{2}$. It follows that the quantity $(-1)^{\operatorname{dim} \mathfrak{m}_{f}^{+}+\operatorname{dim} \mathfrak{k}_{f}^{+}} D_{M_{f}}^{2} / D_{K_{f}}^{2}$ is positive.

- The representation of the compact torus $T_{f}$ on $\mathfrak{u}^{+} / \mathfrak{m}_{f}^{+}$has no fixed vectors (the centralizer of $\mathfrak{b}$ is $\left.\mathfrak{b} \oplus \mathfrak{a}_{0}\right)$ and is the complexification of the real representation on $\mathfrak{n}_{f \mathbb{R}}$.

This means that the eigenvalues of $T_{f}$ on $\mathfrak{u}^{+} / \mathfrak{m}_{f}^{+}$come in complex conjugate pairs; it also shows that $\rho_{U} / \rho_{M_{f}}>0$, being a real-valued character on the connected $\operatorname{group} T_{f}^{\sim}$.

Therefore $D_{U} / D_{M_{f}}>0$.

- The character of $\operatorname{det}\left[1-\mathfrak{p} / \mathfrak{a}_{0}\right]$ is positive because, again, the eigenvalues of $T_{f}$ on $\mathfrak{p} / \mathfrak{a}_{0}$ come in complex conjugate pairs, this being the complexification of $\mathfrak{p}_{\mathbb{R}} / \mathfrak{a}_{0} \cap \mathfrak{p}_{\mathbb{R}}$.

Note that this also implies that

$$
\operatorname{dim} \mathfrak{m}_{f}^{+} \equiv \operatorname{dim} \mathfrak{u}^{+} \text {modulo } 2 .
$$

Since the signs match, it suffices to check (5.5.3) after squaring both sides. In view of (5.5.1) and (5.5.4), our task reduces to that of checking the equality of $T_{f}^{\sim}$-characters: $\operatorname{det}\left[1-\mathfrak{p} / \mathfrak{a}_{0}\right]^{2}=\operatorname{det}\left[1-\mathfrak{u} / \mathfrak{t}_{U}\right] \operatorname{det}\left[1-\mathfrak{m}_{f} / \mathfrak{b}\right] / \operatorname{det}\left[1-\mathfrak{k}_{f} / \mathfrak{b}\right]^{2}$, which in turn would follow from

$$
2\left[\mathfrak{p} / \mathfrak{a}_{0}\right]=\left[\mathfrak{u} / \mathfrak{t}_{U}\right]+\left[\mathfrak{m}_{f} / \mathfrak{b}\right]-2\left[\mathfrak{k}_{f} / \mathfrak{b}\right],
$$

this identity being understood in the Grothendieck group of $T_{f}$-representations.

Now $[\mathfrak{u}]=[\mathfrak{k}]+[\mathfrak{p}]$ as $T_{f}$-representations. Similarly $\left[\mathfrak{m}_{f}\right]=\left[\mathfrak{k} \cap \mathfrak{m}_{f}\right]+\left[\mathfrak{p} \cap \mathfrak{m}_{f}\right]$. So we can write the right-hand side of (5.5.5) as

$$
\begin{aligned}
{\left[\mathfrak{u} / \mathfrak{t}_{U}\right]+\left[\mathfrak{m}_{f} / \mathfrak{b}\right]-2\left[\mathfrak{k}_{f} / \mathfrak{b}\right] } & =[\mathfrak{u}]+\left[\mathfrak{m}_{f}\right]-2\left[\mathfrak{k}_{f}\right]+[\mathfrak{b}]-\left[\mathfrak{t}_{U}\right] \\
& =[\mathfrak{k}]+[\mathfrak{p}]+\left[\mathfrak{k}_{f}\right]+\left[\mathfrak{p} \cap \mathfrak{m}_{f}\right]-2\left[\mathfrak{k}_{f}\right]+[\mathfrak{b}]-\left[\mathfrak{t}_{U}\right] \\
& =\left[\mathfrak{k} / \mathfrak{k}_{f}\right]+\left([\mathfrak{p}]+[\mathfrak{b}]-\left[\mathfrak{t}_{U}\right]\right)+\left[\mathfrak{p} \cap \mathfrak{m}_{f}\right] \\
& =\left[\mathfrak{k} / \mathfrak{k}_{f}\right]+\left[\mathfrak{p} / \mathfrak{a}_{0}\right]+\left[\mathfrak{p} \cap \mathfrak{m}_{f}\right] .
\end{aligned}
$$


Write $\mathfrak{p}_{M}=\mathfrak{p} \cap\left(\mathfrak{m}_{f} \oplus \mathfrak{a}_{0}\right)$. If we show that $\mathfrak{p} / \mathfrak{p}_{M}$ and $\mathfrak{k} / \mathfrak{k}_{f}$ are isomorphic as $T_{f}$-representations, we will be done.

Choose nonzero $X_{0} \in \mathfrak{a}_{0}$ and consider the map

$$
\phi: Y \in \mathfrak{k} / \mathfrak{k}_{f} \mapsto\left[Y, X_{0}\right] \in \mathfrak{p} .
$$

This map is injective, because $\mathfrak{k}_{f}$ exhausts the centralizer of $X_{0}$ in $\mathfrak{k}$; moreover, for any $Z \in \mathfrak{p}_{M}$,

$$
\left\langle\left[Y, X_{0}\right], Z\right\rangle=-\left\langle Y,\left[X_{0}, Z\right]\right\rangle=0,
$$

so the image of $\phi$ in fact lands in $\mathfrak{p}_{M}^{\perp}$. The map $\phi$ thus induces a $T_{f}$-equivariant injective map from $\mathfrak{k} / \mathfrak{k}_{f}$ to $\mathfrak{p} / \mathfrak{p}_{M}$. The difference between the dimensions of these spaces is the difference between the split ranks of $G$ and $M A$; we verify by direct inspection ${ }^{10}$ that this is zero in all cases. These spaces are thus equivariantly isomorphic, as desired.

\subsection{Computation for the fundamental series}

We shall now compute (5.3.3) in the case under consideration, when $Q=P_{f}$ is fundamental and the contragredient representation $\theta_{0}^{*}$ as indexed previously by a dominant character $\mu \in \mathfrak{b}^{*}$.

Let $\left[W_{f} \backslash W_{U}\right]$ be the set of $w \in W_{U}$ such that $\mu_{w}$ is dominant as a weight on $\mathfrak{b}$ (with respect to the roots of $\mathfrak{b}$ on $\mathfrak{k}_{f}$ ), i.e.,

$$
\left[W_{f} \backslash W_{U}\right]=\left\{w \in W_{U}:\left\langle\mu_{w}, \beta\right\rangle \geqslant 0 \text { for all } \beta \in \Delta^{+}\left(\mathfrak{b}, \mathfrak{k}_{f}\right)\right\} .
$$

This is therefore a set of coset representatives for $W_{f}$ in $W_{U}$ : for every such $\beta$,

$$
\left\langle\left(\rho_{U}+\lambda\right), \beta\right\rangle \geqslant\left\langle\rho_{U}, \beta\right\rangle>0 .
$$

This shows that any $\mu_{w}$ lies strictly in the interior of a Weyl chamber for the roots of $\mathfrak{b}$ on $\mathfrak{k}_{f}$.

Let us agree to denote by $\sum^{*}$ any summation over a Weyl group that is weighted by the sign character.

If we equip $T_{f}$ with the Haar measure of total mass 1, the Weyl integration formula formally gives

$$
d(\pi(\theta, v))=\int_{T_{f}} \text { character of }\left(\theta_{0} \otimes \operatorname{det}^{\prime}[1-\mathfrak{p}] \otimes \rho_{\lambda}\right) \frac{\left|D_{K_{f}}\right|^{2}}{\left|W_{f}\right|} d t .
$$

A priori this is only formal; its validity is a nontrivial fact, and only follows from the truth of Blattner's conjecture [37].

We carry out the computation using character formulas for discrete series.

The character of $\rho_{\lambda}$ is given on the maximal torus $T_{U}$ by

$$
\frac{\sum_{w \in W_{U}}^{*} w\left(\rho_{U}+\lambda\right)}{D_{U}} \text {. }
$$

Separately, the numerator and denominator only make sense on $T_{U}^{\sim}$, but the ratio makes sense on $T_{U}$. On the other hand, the character of $\theta_{0}^{*}$ is a distribution whose restriction

${ }^{10}$ For $\mathrm{SL}_{3}(\mathbb{R})$ both ranks are 2 ; for $\mathrm{SO}(p, q)$ both ranks are $\min (p, q)$. 
to $T_{f}$ is given by [39, Theorem 12.7$]$

$$
(-1)^{\frac{1}{2} \operatorname{dim}\left(M_{f} / K_{f}\right)} \frac{\sum_{w \in W_{f}}^{*} w \mu}{D_{M_{f}}} .
$$

Here again the ratio makes sense on $T_{f}$. It thus follows from (5.5.2) that

$$
\begin{aligned}
& d(\pi(\theta, v))=\frac{(-1)^{\frac{1}{2} \operatorname{dim}\left(M_{f} / K_{f}\right)}}{\left|W_{f}\right|} \int_{T_{f}} \overline{\left(\frac{\sum_{w \in W_{f}}^{*} w \mu}{D_{M_{f}}}\right)}\left(\frac{\sum_{w \in W_{U}}^{*} w\left(\rho_{U}+\lambda\right)}{D_{U}}\right) \\
& \operatorname{det}^{\prime}[1-\mathfrak{p}]\left|D_{K_{f}}\right|^{2} d t \\
&=(-1)^{\frac{1}{2} \operatorname{dim}\left(M_{f} / K_{f}\right)+1} \int_{T_{f}} \overline{\left(\sum_{w \in W_{f}}^{*} w \mu\right)}\left(\sum_{w \in\left[W_{f} \backslash W_{U}\right]}^{*} \mu_{w}\right) d t .
\end{aligned}
$$

The equation (5.6.1) implies that $w \mu=\mu_{w^{\prime}}\left(w \in W_{f}, w^{\prime} \in\left[W_{f} \backslash W_{U}\right]\right)$ only if $w$ is trivial; we conclude that

$$
d(\pi(\theta, v))= \begin{cases}(-1)^{\frac{1}{2} \operatorname{dim}\left(M_{f} / K_{f}\right)+1} \operatorname{sgn}(w) & \text { if } \mu=\mu_{w} \text { for some } w \in\left[W_{f} \backslash W_{U}\right] \\ 0 & \text { if not. }\end{cases}
$$

\subsection{The end of the proof of Proposition 5.2.}

The infinitesimal character of $\rho_{\lambda}$ is $\rho_{U}+\lambda$. It thus follows [39, Proposition 8.22 and Lemma 12.28] that

$$
\Lambda_{\rho}-\Lambda_{\pi(\theta, v)}=|\nu|^{2}-|\mu|^{2}+\left|\rho_{U}+\lambda\right|^{2}
$$

Remark. Note that from the definitions, $\left|\rho_{U}+\lambda\right|^{2}-\left|\mu_{w}\right|^{2}$ is always nonnegative. It moreover follows from Parthasarathy's Dirac inequality $[10,58,68]$ that $\rho_{\lambda}$ is strongly acyclic if and only if $\left|\rho_{U}+\lambda\right|^{2}-\left|\mu_{w}\right|^{2}$ is positive for all $w$.

Recall that we assume that $\delta(G)=1$. From this, (5.4.2), (5.2.1), and the result of $\S 5.6$ we get

$$
\begin{aligned}
t_{S}^{(2)}(\rho)= & \left.\frac{(-1)^{\frac{1}{2} \operatorname{dim} N_{f}+\frac{1}{2} \operatorname{dim}\left(M_{f} / K_{f}\right)+1}}{2} c_{S}^{\prime} \sum_{w \in\left[W_{f} \backslash W_{U}\right]} \operatorname{sgn}(w) \frac{d}{d s}\right|_{s=0}\left(\frac{1}{\Gamma(s)} \int_{0}^{\infty} d t\right. \\
& \left.\times \int_{-\infty}^{+\infty} t^{s-1} \mathrm{e}^{-t\left(v^{2}+\left|\rho_{U}+\lambda\right|^{2}-\left|\mu_{w}\right|^{2}\right)} \prod_{\alpha \in \Delta^{+}\left(\mathfrak{t}_{U}, \mathfrak{u}\right)} \frac{\left\langle\mu_{w}+v, \alpha\right\rangle}{\left\langle\rho_{U}, \alpha\right\rangle} \cdot d v\right)
\end{aligned}
$$

It moreover follows from $\S 2.8$ that if $p$ is an even polynomial and $c \geqslant 0$,

$$
\left.\frac{d}{d s}\right|_{s=0} \frac{1}{\Gamma(s)} \int_{0}^{\infty} d t \int_{-\infty}^{+\infty} t^{s-1} \mathrm{e}^{-t\left(y^{2}+c^{2}\right)} p(i y) d y=-2 \pi \int_{0}^{c} p(y) d y .
$$


To conclude, note that $\operatorname{dim} N_{f}+\operatorname{dim}\left(M_{f} / K_{f}\right)=\operatorname{dim} S-1$. Thus we obtain the statement of Proposition 5.2, where the constant $c(S)$ is given by $\frac{\pi}{2} \frac{\left|W_{M_{f}}\right|}{\left|W_{K_{f}}\right|} c_{S}^{\prime}$, and $c_{S}^{\prime}$ is the constant appearing in the Plancherel measure (5.4.2).

\subsection{Nonvanishing in the general case}

Assume that $\delta(S)=1$; we shall show that $t_{S}^{(2)}(\rho)$ is always nonzero and of the sign $(-1)^{\frac{\operatorname{dim} S-1}{2}}$. We follow an idea of Olbrich to minimize computation.

Proof. If $\delta(S)=1$, the (real) Lie algebra of $G$ splits as $\mathfrak{g}_{0} \oplus \mathfrak{g}_{1}$, where $\delta\left(\mathfrak{g}_{0}\right)=0$ and $\mathfrak{g}_{1}$ is isomorphic to either $\mathfrak{s l}_{3}$ or $\mathfrak{s o}_{p, q}$. Using Proposition 5.2 our task can be reduced to that of checking the case where $\mathfrak{g}_{0}$ is trivial, i.e. the (real) Lie algebra of $G$ equals either $\mathfrak{s l}_{3}$ or $\mathfrak{s o}_{p, q}$.

Moreover, the expression $\sum_{w} \int_{v}$ of Proposition 5.2 depends only on the triple $\left(\mathfrak{a}_{0} \subset \mathfrak{t}_{U} \subset \mathfrak{u}\right)$. The isomorphism class of this triple, in the case of $\mathfrak{s o}_{p, q}$, depends only on $p+q$ (more generally, it depends only on the inner form of the Lie algebra).

Our task reduces to that of checking the cases where the real Lie algebra of $G$ is isomorphic to either $\mathfrak{s l}_{3}$ or $\mathfrak{s o}_{p, 1}$. These cases are handled in $\S \S 5.9 .2$ and 5.9.1 respectively.

Proof of Theorem 1.4. The existence of strongly acyclic bundles is proven in $\$ 8.1$.

It follows from (4.1.3), Theorem 4.5, and duality that

$$
\sum^{*} \log \left|H_{j}\left(\Gamma_{N}, M\right)\right| \rightarrow \operatorname{vol}(\Gamma \backslash S) t_{S}^{(2)}(\rho)
$$

as $N$ tends to infinity. The constant $c_{G, M}=t_{S}^{(2)}(\rho)$ has the same sign as $(-1)^{\frac{\operatorname{dim} S-1}{2}}$ as we have just proven, and only depends on $G, M$.

\subsection{Examples.}

5.9.1. $\mathbf{G}=\mathbf{S O}_{2 n+1,1}$. In this case $U \cong \mathrm{SO}_{2 n+2}(\mathbb{R}), K \cong \mathrm{O}_{2 n+1}(\mathbb{R}), M_{f}=K_{f} \cong \mathrm{SO}_{2 n}(\mathbb{R})$ and $S=\mathbb{H}^{2 n+1}$.

In the notation of [11], we may choose a Killing-orthogonal basis $\varepsilon_{i}$ for $\mathfrak{t}_{U}^{*}$ such that:

(1) The positive roots are those roots $\varepsilon_{k} \pm \varepsilon_{l}$ with $1 \leqslant k<l \leqslant n+1$.

(2) $\mathfrak{a}$ is the common kernel of $\varepsilon_{2}, \ldots, \varepsilon_{n}$.

$(3) \mathfrak{b}$ is the kernel of $\varepsilon_{1}$, and the positive roots for $\mathfrak{b}$ on $M_{f} \cap K$ are $\varepsilon_{j} \pm \varepsilon_{k}(1<j<k)$.

(4) The positive roots nonvanishing on $\mathfrak{a}$ are $\varepsilon_{1} \pm \varepsilon_{l}(1<l \leqslant n+1)$, and thus $\alpha_{0}=\varepsilon_{1}$ gives the unique positive restricted root $\mathfrak{a}_{0, \mathbb{R}} \rightarrow \mathbb{R}$.

The representations of $U$ are parametrized by a highest weight $\lambda=\left(\lambda_{1}, \ldots, \lambda_{n+1}\right)=$ $\lambda_{1} \varepsilon_{1}+\cdots+\lambda_{n+1} \varepsilon_{n+1}$ such that $\lambda$ is dominant (i.e. $\lambda_{1} \geqslant \cdots \geqslant \lambda_{n} \geqslant\left|\lambda_{n+1}\right|$ ) and integral (i.e. $\lambda_{i}-\lambda_{i+1} \in \mathbb{Z}, i=1, \ldots, n-1$, and $\lambda_{n} \pm \lambda_{n+1} \in \mathbb{Z}$ ). The action of the Cartan involution on representations is via

$$
\left(\lambda_{1}, \ldots, \lambda_{n+1}\right) \mapsto\left(\lambda_{1}, \ldots, \lambda_{n},-\lambda_{n+1}\right) .
$$


Note in particular (see Lemma 4.1) that $\rho=\rho_{\lambda}$ is strongly acyclic if and only if $\lambda_{n+1} \neq 0$.

The Weyl group of $U$ consists of permutations and sign changes of $\left\{\varepsilon_{1}, \ldots, \varepsilon_{n+1}\right\}$ with even sign (i.e., positive determinant). In particular $\left|W_{U}\right|=2^{n}(n+1)$ !. The subgroup $W_{f}=W_{M_{f}} \subset W_{U}$ comprises all permutations that fix the first coordinate.

We have $\rho_{U}+\lambda=\left(n+\lambda_{1}, n-1+\lambda_{2}, \ldots, \lambda_{n+1}\right)$. We may assume that $\lambda_{n+1} \geqslant 0$ and for convenience we will rewrite the expression as

$$
\rho_{U}+\lambda=\left(a_{n}, \ldots, a_{0}\right) .
$$

Note that $\left(a_{j}\right)$ is a strictly increasing sequence of nonnegative half-integers.

The set $W_{M_{f}} \backslash W_{U}$ has size $2(n+1)$; one may choose a set of representatives for it given by $w_{k}(0 \leqslant k \leqslant n)$, any element sending $\varepsilon_{k}$ to $\varepsilon_{1}$, as well as $w_{k}^{*}$, any element sending $\varepsilon_{k}$ to $-\varepsilon_{1}$. For explicitness we choose $w_{k}$ such that

$$
w_{k}\left(\rho_{U}+\lambda\right)=\left(a_{k}, a_{n}, \ldots, \widehat{a_{k}}, \ldots, a_{0}\right) ;
$$

it is of sign $(-1)^{n-k}$. (As usual, $\widehat{a_{k}}$ denotes that the $a_{k}$ term is omitted.) Choose $w_{k}^{*}$ similarly to $w_{k}$ but replacing $a_{k}, a_{0}$ by $-a_{k},-a_{0}$ respectively. Thus $\mu_{w_{k}}+t \alpha_{0}=$ $\left(t, a_{n}, \ldots, \widehat{a_{k}}, \ldots, a_{0}\right)$.

To apply Proposition 5.2, first note that $\left|\rho_{U}+\lambda\right|^{2}-\left|\mu_{w_{k}}\right|^{2}=a_{k}^{2}|\varepsilon|^{2}$, where $|\varepsilon|^{2}$ is the common value of any $\left|\varepsilon_{j}\right|^{2}$. This again shows that $\rho_{\lambda}$ is strongly acyclic when $a_{0}=\lambda_{n+1} \neq 0$. Set

$$
E=E(\rho)=\prod_{0 \leqslant i<j \leqslant n}\left(a_{j}^{2}-a_{i}^{2}\right), \quad F=\prod_{0 \leqslant i<j \leqslant n}\left(j^{2}-i^{2}\right) .
$$

When $\rho$ is trivial, $E=F$. Now, with the notation as in Proposition 5.2,

$$
P_{w_{k}}\left(t \alpha_{0}\right)=P_{w_{k}^{*}}\left(t \alpha_{0}\right)=\frac{E}{F} \prod_{j \neq k} \frac{t^{2}-a_{j}^{2}}{a_{k}^{2}-a_{j}^{2}} .
$$

Note that the left-hand side is independent of the normalization of the inner product $\langle\cdot, \cdot\rangle$. The polynomial $\Pi_{k}(t)=\prod_{j \neq k} \frac{t^{2}-a_{j}^{2}}{a_{k}^{2}-a_{j}^{2}}$ is of degree $2 n$ and $\Pi_{k}\left( \pm a_{j}\right)=\delta_{j k}$, the Kronecker $\delta$ symbol. Set $Q_{k}=\Pi_{k}+\cdots+\Pi_{n}$; it is the unique even polynomial of degree $\leqslant 2 n$ which satisfies

$$
Q_{k}\left( \pm a_{j}\right)= \begin{cases}0 & \text { if } j<k \\ 1 & \text { if } j \geqslant k\end{cases}
$$

Proposition 5.2 then implies that

$$
\begin{aligned}
t_{\mathbb{H}^{2 n+1}}^{(2)}(\rho) & =(-1)^{n} c\left(\mathbb{H}^{2 n+1}\right) \frac{4|\varepsilon| E}{F} \sum_{k=0}^{n} \int_{0}^{a_{k}} \Pi_{k}(t) d t . \\
& =(-1)^{n} c\left(\mathbb{H}^{2 n+1}\right) \frac{4|\varepsilon| E}{F} \sum_{k=0}^{n} \int_{a_{k-1}}^{a_{k}} Q_{k}(\nu) d \nu .
\end{aligned}
$$

Here we set $a_{-1}=0$. 
Each integral in (5.9.3) is positive. In fact $Q_{k}^{\prime}$ has a root in each interval $\left[ \pm a_{j-1}, \pm a_{j}\right]$ for $1 \leqslant j \neq k$, as well as a root in $\left[-a_{0} ; a_{0}\right]$. Being of degree $\leqslant 2 n-1$, this forces $Q_{k}$ to be either constant (this is the case iff $k=0$ ) or strictly increasing between $a_{k-1}$ and $a_{k}$. It follows that $\int_{a_{k-1}}^{a_{k}} Q_{k}(v) d v>0$.

5.9.2. $\mathbf{G}=\mathrm{SL}_{3}$. In this case $U \cong \mathrm{SU}_{3}, K \cong \mathrm{SO}_{3}, M_{f} \cong\left\{g \in \mathrm{GL}_{2}(\mathbb{R}): \operatorname{det} g= \pm 1\right\}$, and $K_{f} \cong \mathrm{O}_{2}(\mathbb{R})$.

Fix an element $g \in \mathrm{SU}_{3}$ conjugating $\mathfrak{t}_{U}$ into diagonal matrices; let $\varepsilon_{j} \quad(1 \leqslant j \leqslant 3)$ be the pullback, by $g$, of the coordinate functionals. Thus $\sum \varepsilon_{j}=0$; moreover, we may choose $g$ such that:

(1) The positive roots are $\varepsilon_{i}-\varepsilon_{j}$, with $i<j$.

(2) $\rho_{U}=\varepsilon_{1}-\varepsilon_{3}$.

$(3) \mathfrak{b}$ is identified with the kernel of $\varepsilon_{1}+\varepsilon_{2}-2 \varepsilon_{3}$.

(4) $\alpha_{0}=\frac{1}{2}\left(\varepsilon_{1}+\varepsilon_{2}-2 \varepsilon_{3}\right)$ is the unique restricted root $\mathfrak{a}_{0, \mathbb{R}} \rightarrow \mathbb{R}$.

(5) The Weyl group $S_{3}$ acts by permuting the $\varepsilon_{j}$.

Now let $p \geqslant q \geqslant r \in \mathbb{Z}$ and set $\lambda=p \varepsilon_{1}+q \varepsilon_{2}+r \varepsilon_{3}$. Put

$$
\begin{aligned}
A_{1}=\frac{1}{2}(p+1-q), \quad A_{2}=\frac{1}{2}(p-r+2) \quad \text { and } \quad A_{3}=\frac{1}{2}(q-r+1) . \\
C_{1}=\frac{1}{3}(p+q-2 r+3), \quad C_{2}=\frac{1}{3}(p+r-2 q) \quad \text { and } \quad C_{3}=\frac{1}{3}(2 p-q-r+3) .
\end{aligned}
$$

One may choose a set of representatives $w_{k}(k=1,2,3)$ of $W_{M_{f}} \backslash W_{U}$ such that

$$
\mu_{w_{k}}=A_{k}\left(\varepsilon_{1}-\varepsilon_{2}\right) \text { and } \operatorname{sign}\left(w_{k}\right)=(-1)^{k+1} .
$$

Writing $\rho_{U}+\lambda=A_{1}\left(\varepsilon_{1}-\varepsilon_{2}\right)+C_{1} \alpha_{0}$ we see that $\left|\rho_{U}+\lambda\right|^{2}-\left|\mu_{w_{1}}\right|^{2}=C_{1}^{2}\left|\alpha_{0}\right|^{2}$. Similarly we have

$$
\left|\rho_{U}+\lambda\right|^{2}-\left|\mu_{w_{k}}\right|^{2}=C_{k}^{2}\left|\alpha_{0}\right|^{2} \quad \text { and } \quad P_{w_{k}}\left(t \alpha_{0}\right)=A_{k}\left(\frac{9 t^{2}}{4}-A_{k}^{2}\right) \quad(k=1,2,3) .
$$

After a routine computation, Proposition 5.2 therefore gives

$$
t_{S}^{(2)}(\rho)=\left|\alpha_{0}\right| c(S) \sum_{k=1}^{3}(-1)^{k+1} \int_{0}^{\left|C_{k}\right|} A_{k}\left(\left(\frac{9 t^{2}}{4}\right)-A_{k}^{2}\right) d t .
$$

Now $A_{k} \int_{0}^{\left|C_{k}\right|}\left(\frac{9 t^{2}}{4}-A_{k}^{2}\right) d t=\frac{A_{k}\left|C_{k}\right|}{4}\left(3 C_{k}^{2}-4 A_{k}^{2}\right)$. The inner $k$-sum equals

$$
2 A_{1} A_{3} C_{1} C_{3}+2 A_{2}\left|C_{2}\right| \begin{cases}A_{3} C_{3}, & C_{2} \geqslant 0 \\ A_{1} C_{1}, & C_{2} \leqslant 0\end{cases}
$$

which is manifestly positive, since $A_{1}, A_{2}, A_{3}, C_{1}, C_{3}$ are all positive.

5.9.3. Numerical computations. Here we compute the explicit value of the constant $c(S)$ appearing in Proposition 5.2. The computations are not original; they were first done by Olbrich [57] following Harish-Chandra. We just translate these to our setting. 
First recall from $\S 5.7$ that $c(S)=\frac{\pi}{2} \frac{\left|W_{M_{f}}\right|}{\left|W_{K_{f}}\right|} c_{S}^{\prime}$, where $c_{S}^{\prime}$ is the constant appearing in the Plancherel measure (5.4.2). This constant is explicitly computed by Harish-Chandra [36, §24 Theorem 1 and $\S 27$ Theorem 3] but we have to take into account that Harish-Chandra's and our normalizations of the measure $d g$ and $d v$ differ by some multiplicative factor.

Indeed the Plancherel measure (5.4.2) depends on the normalization of the Haar measure $d g$ and of $d v$. Our normalization (cf. §3.3) of $d g$ differ from Harish-Chandra's by the factor $2^{\frac{1}{2}\left(\operatorname{dim}(S)-\operatorname{dim} \mathfrak{a}_{\mathbb{R}}\right)}$ - see [35, $\S 37$ Lemma 2]. Similarly our $d v$ is the Lebesgue measure which corresponds to the metric induced by the $\operatorname{Killing}$ form on $\operatorname{Hom}\left(\mathfrak{a}_{0, \mathbb{R}}, \mathbb{R}\right)$. It differs from Harish-Chandra's by the factor $(2 \pi)^{\delta(G)}$.

We thus get

$$
c_{S}^{\prime}=\frac{1}{\left|W_{A}\right|(2 \pi)^{\frac{1}{2}(\operatorname{dim} G / K+\delta(G))}} \frac{\prod_{\alpha \in \Delta^{+}}\left\langle\rho_{U}, \alpha\right\rangle}{\prod_{\alpha \in \Delta_{\mathfrak{k}^{+}}}\left\langle\rho_{K}, \alpha\right\rangle},
$$

where $W_{A}=\{k \in K: \operatorname{Ad}(k) \mathfrak{a} \subset \mathfrak{a}\} / K_{f}, \Delta_{\mathfrak{k}}^{+}=\Delta^{+}(\mathfrak{b}, \mathfrak{k})$ and $\rho_{K} \in \mathfrak{b}^{*}$ is the half-sum of positive roots in $\Delta_{\mathfrak{k}}^{+}$.

But $[35, \S 37$ Lemma 4] implies that

$$
\frac{\prod_{\alpha \in \Delta^{+}}\left\langle\rho_{U}, \alpha\right\rangle}{\prod_{\alpha \in \Delta_{\mathfrak{k}}^{+}}\left\langle\rho_{K}, \alpha\right\rangle}=(2 \pi)^{\frac{1}{2}\left(\operatorname{dim}\left(U / T_{U}\right)-\operatorname{dim}(K / B)\right)} \frac{\operatorname{vol}(K) \operatorname{vol}\left(T_{U}\right)}{\operatorname{vol}(U) \operatorname{vol}(B)},
$$

where the volumes are the Riemannian ones corresponding to the Killing form.

We may now suppose that $\delta(G)=1$ and let $\alpha_{0}$ be the unique positive restricted root $\mathfrak{a}_{0, \mathbb{R}} \rightarrow \mathbb{R}$. Then $\operatorname{vol}\left(T_{U}\right) / \operatorname{vol}(B)=2 \pi /\left|\alpha_{0}\right|$ and we get

$$
\frac{\prod_{\alpha \in \Delta^{+}}\left\langle\rho_{U}, \alpha\right\rangle}{\prod_{\alpha \in \Delta_{\mathfrak{k}^{+}}}\left\langle\rho_{K}, \alpha\right\rangle}=(2 \pi)^{\frac{1}{2}(\operatorname{dim}(G / K)-1)} \frac{2 \pi}{\left|\alpha_{0}\right| \operatorname{vol}\left(S^{c}\right)} .
$$

We finally verify by direct inspection that $\frac{\left|W_{M_{f}}\right|}{\left|W_{K_{f}}\right|\left|W_{A}\right|}=2$ except for $G=\mathrm{SO}_{2 n+1,1}^{0}$ where $\frac{\left|W_{M_{f}}\right|}{\left|W_{K_{f}}\right|\left|W_{A}\right|}=\frac{1}{2}$. It thus follows from (5.9.4) and (5.9.5) that

$$
c(S)= \begin{cases}\frac{\pi}{\left|\alpha_{0}\right| \operatorname{vol}\left(S^{c}\right)} & \text { if } G \neq \mathrm{SO}_{2 n+1,1}^{0}, \\ \frac{\pi}{4\left|\alpha_{0}\right| \operatorname{vol}\left(S^{c}\right)} & \text { if } G=\mathrm{SO}_{2 n+1,1}^{0} .\end{cases}
$$

Examples. (1) Since the volume of the standard $(2 n+1)$-sphere is $2 \pi^{n+1} / n$ ! we get $c\left(\mathbb{H}^{2 n+1}\right)=\frac{n !}{8\left|\alpha_{0}\right| \pi^{n}}$, so

$$
t_{\mathbb{H}^{2 n+1}}^{(2)}(\rho)=(-1)^{n} \frac{n !}{2 \pi^{n}} \frac{E}{F} \sum_{k=0}^{n} \int_{0}^{a_{k}} \Pi_{k}(t) d t .
$$

(The notation is as in $\S 5.9 .1$.)

When $\rho$ is the trivial representation, we recover that $t_{\mathbb{H}^{3}}^{(2)}=-\frac{1}{6 \pi}, t_{\mathbb{H}^{5}}^{(2)}=\frac{31}{45 \pi^{2}}, \ldots$; see [49] for more values. 
(2) There is a natural Haar measure $d g$ on $\mathrm{SL}_{3}(\mathbb{R})$ which gives covolume $\zeta(2) \zeta(3)$ to $\mathrm{SL}_{3}(\mathbb{Z})$. As in $\S 3.3$, the measure $d g$ is the product of a Riemannian volume form $d x$ on $S=\mathrm{SL}_{3}(\mathbb{R}) / \mathrm{SO}_{3}(\mathbb{R})$ and the Haar measure $d k$ on $K$ normalized such that $\operatorname{vol}(K)=\operatorname{vol}\left(\mathbb{S}^{1}\right) \operatorname{vol}\left(\mathbb{S}^{2}\right)=8 \pi^{2}$. The volume form $d x$ is associated with the invariant metric induced from the trace form of the standard representation of $\mathfrak{s l}_{3}(\mathbb{R})$. Then a routine computation - using e.g. Macdonald's formula [50] - shows that $\operatorname{vol}\left(S^{c}\right)=\pi^{3} / \sqrt{2}$ and we deduce from $\S 5.9 .2$ that

$$
t_{S}^{(2)}(\text { triv })=\frac{1}{\sqrt{2} \pi^{2}}
$$

and so

$$
t_{S}^{(2)}(\operatorname{triv}) \times \operatorname{vol}\left(\mathrm{SL}_{3}(\mathbb{Z}) \backslash S\right)=\frac{\sqrt{2}}{96 \pi^{2}} \zeta(3) \approx 0,001
$$

(3) One of our most important examples is $G=\mathrm{SL}_{2}(\mathbb{C})$. It is locally isomorphic to $\mathrm{SO}_{3,1}$ but we have $U \cong \mathrm{SU}_{2} \times \mathrm{SU}_{2}$ and representations of $U$ are more easily parametrized by two nonnegative integers $(p, q)$; namely,

$$
g_{\theta, \phi}=\left(\begin{array}{cc}
e^{i \theta} & 0 \\
0 & e^{-i \theta}
\end{array}\right) \times\left(\begin{array}{cc}
e^{i \phi} & 0 \\
0 & e^{-i \phi}
\end{array}\right) \mapsto e^{i p \theta+i q \phi} .
$$

The action of the Cartan involution on representations corresponds to the involution $\alpha \leftrightarrow \beta$ on highest weights. The representation $\rho_{\lambda}$ is strongly acyclic if and only if $p \neq q$. The corresponding finite dimensional representation of $\mathrm{SO}_{3,1}^{0}$ is parametrized by $\left(a_{1}, a_{0}\right)=\left(\frac{1}{2}(p+q+2), \frac{1}{2}(p-q)\right)$ and we find

$$
t_{\mathbb{H}^{3}}^{(2)}(\rho)=\frac{-1}{48 \pi}\left\{(p+q+2)^{3}-|p-q|^{3}+3(p+q+2)|p-q|(p+q+2-|p-q|)\right\} .
$$

One interesting possibility is that one could numerically compute the $L^{2}$-torsion sufficiently rapidly for this to be of interest as an algorithm for computing homological torsion. We do not know how efficient one could expect this to be, in practice.

\section{Ash-Serre conjectures and Conjecture 1.3}

We shall discuss the Ash-Serre conjectures, relating torsion classes for arithmetic groups to Galois representations; after this, we explain why these conjectures, together with various known heuristics in number theory, are 'compatible' with Conjecture 1.3. In particular, we shall see how the term $\operatorname{rank}(G)-\operatorname{rank}(K)$ in Conjecture 1.3 arises naturally from arithmetic computations.

This section, by contrast with the foregoing, is very algebraic and should also be regarded as very speculative in character. We do prove, however, two results that are possibly of independent interest: Proposition 6.1 (classification of odd involutions in the $L$-group) and Proposition 6.4 (counting the number of local nearly ordinary representations.)

Proposition 6.1. Let $\mathbf{G}$ be a semisimple simply connected algebraic group over the real numbers. Let ${ }^{L} G$ be the Langlands dual group, i.e. the semidirect 
product $G^{\vee}(\mathbb{C}) \ltimes \operatorname{Gal}(C / \mathbb{R})$, where $G^{\vee}(\mathbb{C})$ is a complex dual group for $\mathbf{G}$ and the action of complex conjugation on $G^{\vee}(\mathbb{C})$ is induced from its action on the Dynkin diagram.

Then every involution in ${ }^{L} G$ lifting the nontrivial element of $\mathrm{Gal}(\mathbb{C} / \mathbb{R})$ has trace $\geqslant \operatorname{rank}(G)-2 \operatorname{rank}(K)$ in the adjoint representation; the set of elements of ${ }^{L} G$ with adjoint trace equal to $\operatorname{rank}(G)-2 \operatorname{rank}(K)$ forms a single $G^{\vee}(\mathbb{C})$-orbit.

This proposition has also been independently proven by Emerton and will appear in a published version of [16].

This motivates the definition of the odd Galois representation that will play a critical role in our later discussion $(\S 6.2 .5)$ : a Galois representation with target ${ }^{L} G$ is odd if the image of complex conjugation lies in the canonical class of involutions specified by the proposition.

Proof. It suffices to verify the assertion of the proposition in the case where the Lie algebra $\mathfrak{g}$ of $\mathbf{G}$ is $\mathbb{R}$-simple and quasi-split (the latter because the $L$-group is unchanged by inner twisting).

Now, the adjoint map induces an action of ${ }^{L} G$ on the Lie algebra $\mathfrak{g}^{\vee}$ of the complex dual group $G^{\vee}(\mathbb{C})$. Since $\mathbf{G}$ is simply connected (and so $G^{\vee}$ is adjoint) the resulting map ${ }^{L} G \rightarrow \operatorname{Aut}\left(\mathfrak{g}^{\vee}\right)$ is injective unless the action of $\operatorname{Gal}(\mathbb{C} / \mathbb{R})$ on $\mathfrak{g}^{\vee}$ is trivial. In all cases, the adjoint map identifies the set of involutions in ${ }^{L} G$ that lift complex conjugation, with the set of involutions of $\mathfrak{g}^{\vee}$ that lie in the inner class of complex conjugation.

We shall now use the fact that involutions correspond to real forms of semisimple Lie algebras together with a case-by-case check. More precisely, if $\mathfrak{h}$ is any complex semisimple Lie algebra, there is a bijection:

$$
\text { involutions of } \mathfrak{h} \text { modulo inner automorphism } \leftrightarrow \text { real forms of } \mathfrak{h} \text {. }
$$

The map from the right-hand to the left-hand side is given by the (conjugacy class of) Cartan involutions associated with a real form. There are natural equivalence relations on both sides: on the left, we have the fibres of the projection to Out(h); on the right, we have the equivalence relation of 'inner twisting', i.e. we regard $\mathfrak{h}_{0}$ and $\mathfrak{h}_{1}$ as equivalent if they are inner forms of one another. These relations are identified under (6.1.1).

Let us recall, moreover, that any real semisimple Lie algebra $\mathfrak{h}_{0}$ possesses a unique quasi-split inner form $\mathfrak{h}_{0}^{q s}$. It is numerically distinguished amongst inner forms of $\mathfrak{h}_{0}$ by the fact that the Cartan involution has minimal trace. ${ }^{11}$

Together with $(6.1 .1)$, this implies that there is a unique $G^{\vee}(\mathbb{C})$-conjugacy class of involutions in ${ }^{L} G$ of minimal trace on $\mathfrak{g}$, but does not identify this trace. Let $\mathfrak{g}_{0}^{\vee}$ be the quasi-split real form of $\mathfrak{g}^{\vee}$ whose associated involution (under the bijection of (6.1.1)) projects, in Out $\left(\mathfrak{g}^{\vee}\right)$, to the image of the complex conjugation under the map $\operatorname{Gal}(\mathbb{C} / \mathbb{R}) \rightarrow \operatorname{Out}(\mathfrak{g})=\operatorname{Out}\left(\mathfrak{g}^{\vee}\right)$.

11 This follows from the Iwasawa 'NAK' decomposition: compare this decomposition for $\mathfrak{h}_{0}$ and its quasi-split form, namely, $\mathfrak{h}_{0}=\mathfrak{n}_{0}+\mathfrak{a}_{0} \oplus \mathfrak{k}_{0}$ and $\mathfrak{h}_{0}^{\mathrm{qS}}=\mathfrak{n}_{0}^{\prime} \oplus \mathfrak{a}_{0}^{\prime} \oplus \mathfrak{k}_{0}^{\prime}$. We wish to show that $\operatorname{dim} \mathfrak{k}_{0}^{\prime} \leqslant \operatorname{dim} \mathfrak{k}_{0}$, or, equivalently, $\operatorname{dim}\left(\mathfrak{n}_{0} \oplus \mathfrak{a}_{0}\right) \leqslant \operatorname{dim}\left(\mathfrak{n}_{0}^{\prime} \oplus \mathfrak{a}_{0}^{\prime}\right)$. But, being quasi-split, $\mathfrak{n}_{0}^{\prime} \oplus \mathfrak{a}_{0}^{\prime}$ complexifies to a Borel subalgebra of $\mathfrak{h}_{0}^{q \mathrm{~s}} \otimes \mathbb{C}$; on the other hand, $\mathfrak{n}_{0} \oplus \mathfrak{a}_{0}$ complexifies to a solvable subalgebra of $\mathfrak{h}_{0} \otimes \mathbb{C}$, necessarily contained in a Borel subalgebra. This proves the desired inequality, and shows that equality holds if and only if $\mathfrak{h}_{0}$ is quasi-split. 
Our assertion now amounts to the following: if $\Theta$ is the involution of $\mathfrak{g}^{\vee}$ obtained by complexifying the Cartan involution of $\mathfrak{g}_{0}^{\vee}$, then

$$
\operatorname{trace}(\Theta)=\operatorname{rank}(G)-2 \operatorname{rank}(K) .
$$

We verify this case by case. The map $\mathfrak{g} \rightarrow \mathfrak{g}_{0}^{\vee}$ is an involution on isomorphism classes of simple real quasi-split Lie algebras; it fixes:

(1) The split forms of $\mathfrak{g}_{2}, \mathfrak{f}_{4}, \mathfrak{e}_{7}, \mathfrak{e}_{8}$ and $\mathfrak{s o}(2 n, 2 n)$. Both sides of $(6.1 .2)$ equal $-\operatorname{rank}(G)$.

(2) $\mathfrak{s o}(2 n-1,2 n+1)$; both sides of $(6.1 .2)$ equal $2-2 n$.

(3) Every $\mathfrak{g}$ that is not absolutely simple, i.e., any complex simple Lie algebra, considered as a real Lie algebra; in those cases, both sides of (6.1.2) are 0.

As for the remaining cases, the involution switches pairs of rows in the table

\begin{tabular}{|c|c|c|}
\hline $\mathfrak{g}$ & $r_{G}-2 r_{K}$ & $\operatorname{trace}(\Theta)$ \\
\hline $\mathfrak{s u}(p, p)$ & $-2 p$ & 0 \\
$\mathfrak{s l}_{2} p$ & 0 & $-2 p$ \\
\hline $\mathfrak{s u}(p, p+1)$ & $-2 p-1$ & 1 \\
$\mathfrak{s l}_{2 p+1}$ & 1 & $-2 p-1$ \\
\hline $\mathfrak{s o}(p, p+1)$ & $-p$ & $-p$ \\
$\mathfrak{s p}(2 p)$ & $-p$ & $-p$ \\
\hline $\mathfrak{s o}(2 p+1,2 p+1)$ & $1-2 p$ & $-1-2 p$ \\
$\mathfrak{s o}(2 p, 2 p+2)$ & $-1-2 p$ & $1-2 p$ \\
\hline $\mathfrak{e}_{6}^{\mathrm{split}}$ & -2 & -6 \\
$\mathfrak{e}_{6}^{\mathrm{qs}}$ & -6 & -2 \\
\hline
\end{tabular}

In the last line, $\mathfrak{e}_{6}^{\mathrm{qs}}$ denotes the unique quasi-split and nonsplit form of $\mathfrak{e}_{6}$.

\subsubsection{Examples.}

(1) For $\mathbf{G}=\mathrm{SL}_{n}$, an odd involution on $\mathfrak{g}^{\vee}=\mathfrak{s l}_{n}$ is conjugation with

$$
\pm\left(\begin{array}{ccccc}
1 & & & & \\
& -1 & & & \\
& & 1 & & \\
& & & -1 & \\
& & & & \ddots
\end{array}\right) \text {. }
$$

(2) For $\mathbf{G}=\mathrm{SU}_{n}$, an odd involution on $\mathfrak{g}^{\vee}=\mathfrak{s l}_{n}$ is represented by $X \mapsto-X^{t}$ (negative transpose).

(3) If $\mathbf{G}$ admits discrete series (i.e., $\operatorname{rank}(G)=\operatorname{rank}(K)$ ), then the odd involution is the unique conjugacy class with trace $-\operatorname{rank}(G)$. This case is discussed in detail by Gross [32]. 
(4) If $\mathbf{G}_{1}$ is a complex reductive group, and $\mathbf{G}$ the Weil restriction of scalars to $\mathbf{R}$, then an odd involution $\mathfrak{g}^{\vee}=\mathfrak{g}_{1}^{\vee} \oplus \mathfrak{g}_{1}^{\vee}$ is given by $(X, Y) \mapsto(Y, X)$.

\subsection{The modularity conjecture}

We formulate a slight generalization of [2] (a split simply connected semisimple group, as opposed to $\mathrm{SL}_{n}$ ); we do not address the difficult question of pinning down all the weights in which a given Galois representation occurs (see e.g. [13,28,38] and an unpublished manuscript by Clozel and Belabas where they make precise computations for $\mathrm{SL}_{2}$ over an imaginary quadratic field).

The assumption of being simply connected is to avoid a host of difficulties that occur, even concerning characteristic 0 Galois representations, in the general case. For a detailed treatment we refer the reader to the paper [14].

6.2.1. Let $\mathbf{G}$ be a semisimple simply connected split $^{12}$ algebraic group over $\mathbb{Z}$. Let $G^{\vee}$ be the dual group. We regard it as a split group over $\mathbb{Z}$.

For later use, we fix a maximal torus $\mathbf{T} \subset \mathbf{G}$, a Borel $\mathbf{B} \subset \mathbf{G}$, and thus a system of positive roots for $\mathbf{T}$. Because $G$ is simply connected, the half-sum of positive roots gives a character $\rho: \mathbf{T} \rightarrow \mathbb{G}_{m}$.

We also fix a torus $T^{\vee} \subset G^{\vee}$ dual to $\mathbf{T}$, and a Borel $B^{\vee}=T^{\vee} N^{\vee} \subset G^{\vee}$ containing $T^{\vee}$.

6.2.2. Define the level $Q$ subgroup $\Gamma(Q)=\operatorname{ker}(\mathbf{G}(\mathbb{Z}) \rightarrow \mathbf{G}(\mathbb{Z} / Q \mathbb{Z}))$. Let $\Gamma_{0}(Q)$ be the preimage of $\mathbf{B}(\mathbb{Z} / Q \mathbb{Z})$ under the same map.

6.2.3. We define a weight to be an algebraic character $\mathbf{x}$ of $\mathbf{T}$ such that $\mathbf{x}-\rho$ is dominant. We say that it is strongly regular if $\left\langle\mathbf{x}, \alpha^{\vee}\right\rangle \geqslant 2$ for every simple coroot $\alpha^{\vee}$. In what follows, we always suppose that $\mathbf{x}$ is strongly regular.

6.2.4. We shall extract from $\mathrm{x}$ an arithmetic $\Gamma$-module $M_{\mathrm{x}}$.

Being dominant, $\mathbf{x}-\rho$ is the highest weight of an irreducible representation $\alpha: \mathbf{G}_{\mathbb{Q}} \rightarrow \mathrm{GL}(W)$, where $W$ is a finite dimensional $\mathbb{Q}$-vector space. We choose a $\Gamma$ (1)-stable $\mathbb{Z}$-lattice $M_{\mathbf{x}} \subset W$. Such a lattice is not, in general, unique.

6.2.5. Fix an algebraic closure $\overline{\mathbb{Q}}$ of $\mathbb{Q}$ and a place of $\overline{\mathbb{Q}}$ above every place $v$ of $\mathbb{Q}$. This gives an algebraic closure $\overline{\mathbb{Q}_{v}}$ of $\mathbb{Q}_{v}$ and an embedding $D_{v}:=\operatorname{Gal}\left(\overline{\mathbb{Q}_{v}} / \mathbb{Q}_{v}\right) \hookrightarrow \operatorname{Gal}(\overline{\mathbb{Q}} / \mathbb{Q})$. We henceforth regard $D_{v}$ as a subgroup of $\operatorname{Gal}(\overline{\mathbb{Q}} / \mathbb{Q})$. Let $I_{v} \subset D_{v}$ be the inertia group for $v$ finite. For $v$ infinite, let $c_{\infty}$ be the nontrivial element of $D_{v}$.

6.2.6. Let $k$ be a finite field of characteristic $p$ and of cardinality $q$.

Let $\omega: I_{p} \subset D_{p} \rightarrow \mathbb{F}_{p}^{\times} \hookrightarrow \mathbb{G}_{m}(k)$ be the cyclotomic character (i.e., the map $D_{p} \rightarrow \mathbb{F}_{p}^{\times}$ arises from the action on $p$ th roots of unity). Define the character associated with $\mathbf{x}$ :

$$
\psi_{\mathrm{x}}: I_{p} \rightarrow T^{\vee}(k)
$$

as the composition of $\omega$ with the dual $\mathbf{x}^{\vee}: \mathbb{G}_{m} \rightarrow T^{\vee}$.

12 The assumption of being split is not necessary; we have imposed it to simplify the notation at several points. The main goal of this section is to understand the arithmetic significance of the factor $\operatorname{rank}(G)-\operatorname{rank}(K)$, and this is already interesting in the split case. 
6.2.7. A representation $\sigma_{v}: D_{v} \rightarrow G^{\vee}(k)$ is said to be:

(1) $(v=\infty) O d d$, if $\sigma\left(c_{\infty}\right)$ has trace $\operatorname{rank}(G)-2 \operatorname{rank}(K)$ in the adjoint action on $\mathfrak{g}$.

(2) $(v=p)$ Nearly ordinary of type $\mathbf{x}$, if it is conjugate to a representation with image in $B^{\vee}(k)$, which, upon restriction to inertia and projection to $B^{\vee} / N^{\vee}(k) \cong T^{\vee}(k)$, equals $\psi_{\mathbf{x}}$.

We will sometimes also apply the description nearly ordinary at $\mathbf{x}$ to a representation with codomain $B^{\vee}(k)$; this means simply that the projection to $B^{\vee} / N^{\vee}$ coincides, on inertia, with $\psi_{\mathrm{x}}$.

(3) $(v=\ell \neq p)$ Steinberg at $\ell$ if its restriction to $I_{\ell}$ has as image the cyclic subgroup generated by a principal unipotent element ${ }^{13}$ of $G^{\vee}(k)$. Since $I_{\ell}$ has a unique cyclic quotient of order $p$, and all principal unipotent elements in $G^{\vee}(k)$ are conjugate, there exists only one conjugacy class of such maps, $I_{\ell} \rightarrow G^{\vee}(k)$.

Let $\sigma: \operatorname{Gal}(\overline{\mathbb{Q}} / \mathbb{Q}) \rightarrow G^{\vee}(k)$ be a homomorphism. We say that it is odd (resp. nearly ordinary of type $\mathbf{x}$, Steinberg at $\ell$ ) if its restriction to $D_{v}$ for $v=\infty(\operatorname{resp} . v=p, v=\ell)$ is.

Conjecture 6.3. Let $S$ be a finite subset of places of $\mathbb{Q}$, not containing p. Suppose $\rho: \mathrm{Gal} \rightarrow G^{\vee}(k)$ is an odd representation, nearly ordinary of type $\mathbf{x}$, Steinberg at every place in $S$, and unramified outside $S$. Put $N=\prod_{\ell \in S} \ell$.

Then there is a Hecke eigenclass in $H^{*}\left(\Gamma_{0}(N), M_{\mathbf{x}}\right)$ that matches $\rho$.

The meaning of 'matches' is the usual compatibility ${ }^{14}$ between Frobenius eigenvalues and Hecke eigenvalues, which we require only at primes not dividing $N p$. The idea of such a conjecture is due to Ash and others and was formulated precisely for $\mathbf{G}=\mathrm{SL}_{n}$ in [2]. However, to our knowledge, it has not been formulated for a general group G; any error in this formulation is due solely to us. We have formulated it only in the special case of Steinberg ramification at $S$ so that we could be precise about the level (in particular, to obtain a numerically falsifiable conjecture!)

Suppose, for example, that $\mathbf{G}=\mathrm{SL}_{n}$; take $\mathbf{T}$ to be the diagonal torus. For any integers $a_{1} \geqslant a_{2} \geqslant \cdots$,

$$
\mathbf{x}_{a_{1} a_{2} \ldots a_{n}}:\left(\begin{array}{cccc}
y_{1} & 0 & \ldots & 0 \\
0 & y_{2} & \ldots & 0 \\
0 & 0 & \ddots & 0 \\
0 & 0 & \ldots & y_{n} .
\end{array}\right) \mapsto \prod y_{i}^{a_{i}+n-i}
$$

is a weight. It is strongly regular if $a_{1}>a_{2}>\cdots>a_{n}$. A representation $\rho$ : $\operatorname{Gal}\left(\overline{\mathbb{Q}_{p}} / \mathbb{Q}_{p}\right) \rightarrow \mathrm{PGL}_{n}(k)$ is nearly ordinary of type $\mathbf{x}_{a_{1}, \ldots, a_{n}}$ if its restriction to inertia

13 For the purpose of this definition, we shall suppose that $\ell$ is a 'good prime' for $G^{\vee}$.

14 To be precise: Let $s$ be a prime number distinct from $p$. Let $\mathscr{H}_{s}$ be the 'abstract' algebra over $\mathbb{Z}$ generated by Hecke operators at $s$, i.e. the algebra of $\mathbb{Z}$-valued $\mathbf{G}\left(\mathbb{Z}_{S}\right)$-bi-invariant compactly supported functions on $\mathbf{G}\left(\mathbb{Q}_{s}\right)$. Then the usual definition of the Satake isomorphism (see [33] for discussion) actually defines an isomorphism $\mathscr{H}_{S} \otimes k \rightarrow \operatorname{Rep}(\check{G}) \otimes_{\mathbb{Z}} k$; it is important that $\mathbf{G}$ is simply connected for this remark - otherwise the half-sum of positive roots causes difficulty. 
is conjugate to

$$
\left(\begin{array}{cccc}
\omega^{a_{1}+n-1} & * & \ldots & * \\
0 & \omega^{a_{2}+n-2} & \ldots & * \\
0 & 0 & \ddots & * \\
0 & 0 & \ldots & \omega^{a_{n}} .
\end{array}\right)
$$

where $\omega: I_{p} \rightarrow \mathbb{Z}_{p}^{\times}$is the cyclotomic character.

For $n=2$, the representation of $\mathbf{G}=\mathrm{SL}_{2}$ with highest weight $\mathbf{x}-\rho$ is the symmetric $\left(a_{1}-a_{2}\right)$ th power of the standard representation; thus, this corresponds to modular forms of weight $a_{1}-a_{2}+2$, and strong regularity corresponds to weight 3 or greater.

The following result is perhaps of independent interest; it will be of use in $\S 6.5 .1$.

Proposition 6.4. Suppose $\mathbf{x}$ to be strongly regular. For sufficiently large $p$, the number of nearly ordinary representations $\operatorname{Gal}\left(\overline{\mathbb{Q}_{p}} / \mathbb{Q}_{p}\right) \rightarrow G^{\vee}(k)$ of type $\mathbf{x}$ equals $\left|G^{\vee}(k)\right| \cdot\left|N^{\vee}(k)\right|$.

The condition sufficiently large $p$ arises in one way as follows: we wish to guarantee, for certain $m$ depending on $\mathbf{x}$, that the $m$ th power of the cyclotomic character $I_{p} \rightarrow \mathbb{F}_{p}^{*}$ is nontrivial. This will be so if $p-1>m$, but not in general if $p$ is small.

Let us formulate an alternative phrasing, which will be convenient in the proof: let $G_{1}, G_{2}$ be finite groups, and let $\operatorname{Hom}\left(G_{1}, G_{2}\right)^{\sharp}$ be the set of homomorphisms up to $G_{2}$-conjugacy. Then $\operatorname{Hom}\left(G_{1}, G_{2}\right)^{\sharp}$ is endowed with a natural measure: the $G_{2}$-conjugacy class of a homomorphism $\alpha$ has mass $\frac{1}{|Z(\alpha)|}$; here $Z(\alpha)$ is the centralizer of $\alpha$ in $G_{2}$. Alternatively we could say that the measure (or mass) of $S \subset \operatorname{Hom}\left(G_{1}, G_{2}\right)^{\sharp}$ is the number of preimages in $\operatorname{Hom}\left(G_{1}, G_{2}\right)$ divided by $\left|G_{2}\right|$. With these conventions, we may rephrase the proposition: the mass of nearly ordinary representations of type $\mathbf{x}$ equals $\left|N^{\vee}(k)\right|$.

Proof. Write, as shorthand, $D_{p}=\operatorname{Gal}\left(\overline{\mathbb{Q}_{p}} / \mathbb{Q}_{p}\right)$. Fix an algebraic closure $\bar{k}$ of $k$.

Let $\psi_{1}, \psi_{2}$ be any two nearly ordinary representations of type $\mathbf{x}$ valued in $B^{\vee}(k)$. We claim that if the $\psi_{i}$ are $G^{\vee}(k)$-conjugate, then they are already $B^{\vee}(k)$-conjugate. Suppose in fact that $\operatorname{Ad}(g) \psi_{1}=\psi_{2}$ for some $g \in G^{\vee}(k)$. The intersection $g B^{\vee} g^{-1} \cap B^{\vee}$ contains a maximal torus $T^{\vee^{\prime}}$. Let $N^{\prime}$ be the normalizer of $T^{\vee^{\prime}}$. All Borels containing a torus are conjugate under the normalizer of that torus, so there exists $n^{\prime} \in N^{\prime}(\bar{k})$ such that $g B^{\vee} g^{-1}=n^{\prime} B^{\vee} n^{\prime-1}$; since $B^{\vee}$ is its own normalizer, it follows that there exists $b \in B^{\vee}(\bar{k})$ such that $g=n^{\prime} b$.

The inclusion into $B^{\vee}$ induces an isomorphism of $T^{\vee \prime}$ with $B^{\vee} / N^{\vee}$. Let $w$ be the automorphism of the latter group induced by $n^{\prime}$. The composition

$$
I_{p} \hookrightarrow D_{p} \stackrel{\psi_{j}}{\longrightarrow} B^{\vee}(k) \rightarrow\left(B^{\vee} / N^{\vee}\right)(k)
$$

is independent of $j$ (it is specified in terms of $\mathbf{x}$ ). It is also $w$-fixed. If $w$ is a nontrivial element of the Weyl group, this contradicts strong regularity for $q$ large.

Thus, $n^{\prime} \in B^{\vee}(\bar{k})$, which shows that $g \in B^{\vee}(k)$. 
Our task is reduced to that of proving that the total mass of nearly ordinary representations of type $\mathbf{x}$ from $D_{p}$ to $B^{\vee}(k)$ equals $\left|N^{\vee}(k)\right|$; equivalently, the number of nearly ordinary representations $D_{p} \rightarrow B^{\vee}(k)$ equals $\left|N^{\vee}(k)\right| \cdot\left|B^{\vee}(k)\right|$.

Let $\psi: D_{p} \rightarrow T^{\vee}(k)$ be any character extending $\psi_{\mathrm{x}}: I_{p} \rightarrow T^{\vee}(k)$. All such $\psi$ are unramified twists of one another; the total number of such $\psi$, then, equals $\left|T^{\vee}(k)\right|$.

On the other hand, nearly ordinary representations $D_{p} \rightarrow B^{\vee}(k)$ are precisely lifts (with respect to the isomorphism $\left.B^{\vee}(k) / N^{\vee}(k) \cong T^{\vee}(k)\right)$ of some such $\psi$. Our task therefore reduces to that of showing that the total number of lifts of any such $\psi: D_{p} \rightarrow T^{\vee}(k)$ equals $\frac{\left|N^{\vee}(k)\right| \cdot\left|B^{\vee}(k)\right|}{\left|T^{\vee}(k)\right|}=\left|N^{\vee}(k)\right|^{2}$.

Now consider the lower central series for $N^{\vee}=U_{0} \supset U_{1} \supset U_{2} \supset \cdots$. Thus $B^{\vee} / U_{0}(k)=$ $T^{\vee}(k)$. Suppose that we have fixed a lift $\psi_{j}$ of $\psi$ to $B^{\vee} / U_{j}(k)$. We have an exact sequence of algebraic groups

$$
U_{j} / U_{j+1} \rightarrow B^{\vee} / U_{j+1} \rightarrow B^{\vee} / U_{j}
$$

This sequence is also exact on $k$-points. The group $X_{j}=U_{j}(k) / U_{j+1}(k)$ is abelian; $D_{p}$ acts on it (via conjugation composed with $\psi_{j}$ ) and the resulting action decomposes into characters of the form $\alpha \circ \psi_{j}$, for various roots $\alpha: T^{\vee} \rightarrow k^{*}$.

By the assumption of strong regularity, no $\alpha \circ \psi_{j}$ is either trivial or cyclotomic, at least for sufficiently large $p$. Therefore, $H^{0}\left(D_{p}, X_{j}\right)=H^{2}\left(D_{p}, X_{j}\right)=0$. The vanishing of $H^{2}$ shows that $\psi_{j}$ lifts to $B^{\vee} / U_{j+1}$; the vanishing of $H^{0}$ proves that $U_{j} / U_{j+1}$ acts freely by conjugation on the set of lifts. The number of such lifts up to $U_{j} / U_{j+1}$-conjugacy is $H^{1}\left(D_{p}, X_{j}\right)=\left|X_{j}\right|$, by the local Euler characteristic formula [56]; the total number of lifts is therefore $\left|X_{j}\right|^{2}$.

The desired conclusion follows by iteration.

\subsection{Bhargava's heuristics}

This section is rather speculative in nature. Our goal is to verify that Conjecture 6.3 is compatible with the main result of this paper, Theorem 1.4. In other terms: does there exist a sufficiently large supply of Galois representations to account for the (proven) exponential growth in torsion, when $\delta=1$ ?

Current technology is wholly inadequate to answer this question. We shall use heuristics for counting number fields proposed by Bhargava. These suggest - as we shall explain - that the fraction of squarefree levels $N$ for which there is an eigenclass in $H^{*}\left(\Gamma(N), M_{\mathbf{x}} \otimes \mathbb{F}_{p}\right)$ is $\gg p^{-\delta(G)}$. Suppose now that $M_{\mathbf{x}}$ is strongly acyclic; since the product $\prod_{p} p^{p^{-\delta(G)}}$ diverges when $\delta(G)=1$, this and Conjecture 6.3 imply the existence of levels $N$ for which the torsion group has unbounded size. This is indeed compatible with the conclusion of Theorem 1.4.

It is also possible to obtain support for Conjecture 1.3 via similar reasoning, but we do not discuss this here, focusing only on the case $\delta=1$ where we can prove unconditional results about torsion.

6.5.1. Fix $\mathcal{Q}$ a finite group.

Let $\mathbf{L}=\prod_{v} \operatorname{Hom}\left(D_{v}, \mathcal{Q}\right)^{\sharp}$ (the restricted product, taken with respect to unramified representations). 
Let $\mathbf{G}$ be the $\mathcal{Q}$-conjugacy classes of surjective homomorphisms $\operatorname{Gal}(\overline{\mathbb{Q}} / \mathbb{Q}) \rightarrow \mathcal{Q}$.

Thus there is a natural map $\mathbf{G} \rightarrow \mathbf{L} .{ }^{15}$

The 'mass' (see the remarks after Proposition 6.4) defines a measure on $\operatorname{Hom}\left(D_{v}, \mathcal{Q}\right)^{\sharp}$. For $v \neq \infty$, the set of unramified homomorphisms has mass 1 . Thus, this gives rise to a product measure $\mu$ on $\mathbf{L}$. Roughly speaking, Bhargava's heuristic suggests that the expected number of elements of $\mathbf{G}$ inside a large subset $S \subset \mathbf{L}$ should be approximately $\mu(S)$.

Bhargava formulates this as a conjecture only in the following specific case: for $\mathcal{Q}=S_{n}$, we can define the 'discriminant' of any element of $\mathbf{G}$, i.e., the discriminant of the associated degree $n$ extension. This map $\mathbf{G} \rightarrow \mathbb{N}_{\geqslant 1}$ factors through $\mathbf{L}$. Let $\mathbf{L}(N) \subset \mathbf{L}, \mathbf{G}(N) \subset \mathbf{G}$ be the subsets of discriminant $\leqslant N$. Then Bhargava conjectures that

$$
|\mathbf{G}(N)| \sim \mu(\mathbf{L}(N)) \quad N \rightarrow \infty .
$$

Bhargava proves this conjecture for $n \leqslant 5$. If we move away from $\mathcal{Q}=S_{n}$, there is no shortage of simple examples (e.g., $\mathcal{Q}=S_{3}^{3}$ ) where the heuristic fails in the precise form above. Our goal, however, is only to use it as an indication of the rough order of magnitude.

6.5.2. Return to the setting of Conjecture 6.3. Let $S$ be a finite subset of places of $\mathbb{Q}$ not containing $p$, and let $N(S)$ be the corresponding squarefree integer - a product of the primes in $S$. We are interested in the number $A(S)$ of Galois representations $\rho: \operatorname{Gal}(\overline{\mathbb{Q}} / \mathbb{Q}) \rightarrow G^{\vee}(k)$ as in Conjecture 6.3 - or, rather, the behaviour of $A(S)$ on average with respect to $S$.

For $v=\infty, p, \ell \neq p$ respectively, we define $M(v) \subset \operatorname{Hom}\left(D_{v}, G^{\vee}(k)\right)^{\sharp}$ to consist of (respectively) odd, nearly ordinary of type $\mathbf{x}$, and Steinberg-at- $\ell$ maps. Let

$$
\mu(S)=\operatorname{mass} M(\infty) \cdot \operatorname{mass} M(p) \cdot \prod_{\ell \in S} \operatorname{mass} M(\ell) .
$$

Now, Bhargava's heuristics suggest that

$$
\sum_{N(S)<X} A(S) \sim \sum_{N(S)<X} \mu(S) .
$$

We claim that $\mu(S)=q^{-\delta(G)}\left(1+O\left(q^{-1 / 2}\right)\right)$ for any such $S$; this follows from the equality $2 \operatorname{dim}(N)+\operatorname{rank}(K)-\operatorname{dim}(G)=-\delta(G)$ and the following remarks:

(1) For $\ell \neq p$ a finite prime, mass $M(\ell)=1$ : the Steinberg-at- $\ell$ maps form a single conjugacy class after restriction to $I_{\ell}$.

(2) Let $\mathbf{O}$ be the algebraic subvariety of $G^{\vee}$ consisting of odd involutions. We regard $\mathbf{O}$ as a scheme over $\mathbb{Z}$. It follows from Proposition 6.1 that $\mathbf{O} \times \mathbb{Z} \mathbb{C}$ is an irreducible variety of dimension $\operatorname{dim}(N)+\operatorname{rank}(K) .{ }^{16}$ By the Lang-Weil

15 The letters $G$ and $L$ stand for global and local.

16 Indeed, Proposition 6.1 shows that the complex points of $\mathbf{O} \times \mathbb{Z} \mathbb{C}$ form a single $G^{\vee}(\mathbb{C})$ orbit, and the dimension $D$ of the Lie algebra of the stabilizer of any point $\Theta$ satisfies $2 D-\operatorname{dim}(G)=\operatorname{trace}(\Theta)$. We get $D=\frac{\operatorname{dim}(G)+\operatorname{rank}(G)-2 \operatorname{rank}(K)}{2}$ and the claim follows. 
estimates, $|\mathbf{O}(k)|=q^{\operatorname{dim}(N)+\operatorname{rank}(K)}\left(1+O\left(q^{-1 / 2}\right)\right)$. We conclude that

$$
\operatorname{mass}(M(\infty))=q^{\operatorname{dim}(N)+\operatorname{rank}(K)-\operatorname{dim}(G)}\left(1+O\left(q^{-1 / 2}\right)\right)
$$

(3) For $v=p$, Proposition 6.4 gives $\operatorname{mass}(M(p))=q^{\operatorname{dim}(N)}$.

We conclude, then, that - if we suppose valid a generalization of Bhargava's heuristics - the average value of $A(S)$, over $S$ with $N(S)<X$, approaches $q^{-\delta(G)}\left(1+O\left(q^{-1 / 2}\right)\right.$ ). As we have already discussed at the start of this section, this is 'compatible' with the conclusion of Theorem 1.4 in the case $\delta=1$. It also suggests that there should be a 'paucity' of Steinberg torsion classes when $\delta \geqslant 2$.

Remark. One can also try to analyze the issue of torsion via eigenvarieties or Galois deformations. These (roughly speaking) parametrize lifts of a given $\bmod p$ torsion class or Galois representation, and conjectures concerning their dimension are therefore related to the likelihood that a $\bmod p$ class will lift modulo $p^{2}$. In our (limited and possibly mistaken) understanding, conjectures such as those of $[16,19]$ suggest that the 'likelihood' that a given class modulo $p$ lifts modulo $p^{2}$ should be of order $p^{-\delta(G)}$. Also, Conjecture 6.1 of [16] also suggests that, when $\delta=1$, the torsion is largely in 'the middle dimension', namely, in degree $\frac{\operatorname{dim}(S)-1}{2}$.

\section{The combinatorial picture}

This section has a different flavour to the rest of the paper; it is more general, in that we deal with an arbitrary cell complex; however, it is also less general in that we deal with a tower of coverings of a fixed space, whereas many of our previous results applied to any sequences of locally symmetric spaces with increasing injectivity radius.

We have seen in $\S 2.5$ that 'limit multiplicity formulas' may be easier to prove in the context of combinatorial torsion than from the 'de Rham' perspective. Such a phenomenon already appears in the classical case of normalized Betti numbers converging toward $L^{2}$-Betti numbers. The most general result, due to Lück [48], is proved in the combinatorial setting and no general proof is known in the analytical one.

\section{1 .}

From now on let $K$ be a path-connected finite cell complex having a universal cover $\widetilde{K}$ and fundamental group $\Gamma$, such that $K$ is the quotient of $\widetilde{K}$ by (left) deck transformations. By 'the cover of $K$ corresponding to' a subgroup $\Gamma^{\prime} \leqslant \Gamma$, we mean the quotient $\widetilde{K} / \Gamma^{\prime}$ together with the induced map to $K$.

Now let $\Lambda$ be a quotient of $\Gamma$, i.e., we regard it as being equipped with a map $\Gamma \rightarrow \Lambda$. Let $\widehat{K}$ be the covering space of $K$ corresponding to the kernel of $\Gamma \rightarrow \Lambda$; let $\Lambda_{N} \subset \cdots \subset \Lambda_{1}=\Lambda$ be a decreasing sequence of finite index normal subgroups, with $\bigcap_{N=1}^{\infty} \Lambda_{N}=1$, and $K_{N}$ the cover corresponding to the preimage of $\Lambda_{N}$ in $\Gamma$. Denote by

$$
T\left(K_{N}\right)=\prod^{*}\left|H^{i}\left(K_{N}\right)_{\text {tors }}\right|
$$

the torsion part of the cohomology, and define the regulator $R^{i}\left(K_{N}\right)$ as in (2.2.4). 
Let $C^{j}(\widehat{K})$ be the cellular cochain complex of $\widehat{K}$. Each $C^{j}$ is a finitely generated free $\mathbb{Z}[\Lambda]$-module. A cell complex for $K_{N}$ is given by $C^{j}(\widehat{K}) \otimes_{\mathbb{Z} \Lambda} \mathbb{Z}\left[\Lambda / \Lambda_{N}\right]$. In all cases, we equip the complexes with the inner products in which the characteristic functions of distinct cells form an orthonormal basis.

Let $\Delta_{j}=d_{j-1} d_{j-1}^{*}+d_{j}^{*} d_{j}$; here $d_{j}^{*}$ is the adjoint of $d_{j}$ with respect to the inner product described. Each $\Delta_{j}$ is a $\mathbb{Z}[\Lambda]$-endomorphism of the finite free $\mathbb{Z}[\Lambda]$-module $C^{j}$.

In particular, if we fix a basis for $C^{j}$ as a left $\mathbb{Z}[\Lambda]$-module, $\Delta_{j}$ is given by right multiplication by a unique matrix with entries in $\mathbb{Z}[\Lambda]$. We do indeed fix such bases, and, in what follows, regard $\Delta_{j}$ as an element of $\operatorname{Mat}_{n_{j}}(\mathbb{Z}[\Lambda])$.

\section{2.}

Let us discuss, first of all, the simple case where $\Lambda=\mathbb{Z}^{m}, \Lambda_{N}=N \mathbb{Z}^{m}$.

$\ell^{2}$-acyclicity.. For $\left(z_{1}, \ldots, z_{m}\right) \in\left(\mathbb{C}^{\times}\right)^{m}$, let $\Delta_{j}(z) \in \operatorname{Mat}_{n_{j}}(\mathbb{C})$ be obtained from $\Delta_{j}$ via the homomorphism $\left(n_{1}, \ldots, n_{m}\right) \in \Lambda \mapsto \prod_{i} z_{i}^{n_{i}}$. We say that $\widehat{K}$ is $\ell^{2}$-acyclic if $\operatorname{det} \Delta_{j}(z)$ - which is always a polynomial in $z_{i}^{ \pm 1}-$ is not identically vanishing for any $j$. (This condition is equivalent to the vanishing of the reduced $\ell^{2}$-cohomology.)

Note that

$$
\operatorname{dim} H^{j}\left(K_{N}, \mathbb{C}\right)=\sum_{\mathbf{z}^{N}=1} \operatorname{dim} \operatorname{ker} \Delta_{j}(z) .
$$

The set of roots of unity contained in the zero locus of ker $\Delta_{j}$ is contained in a finite union of translates of proper subtori of $\left(S^{1}\right)^{m}$ (this is a simple example of a 'Manin-Mumford' phenomenon; see [23] for a discussion of this in topological context) so it follows that, if $\widehat{K}$ is $\ell^{2}$-acyclic, then

$$
\operatorname{dim} H^{j}\left(K_{N}, \mathbb{C}\right) \leqslant A N^{m-1},
$$

the constant $A$ depending only on $K$.

$\ell^{\mathbf{2}}$-torsion.. We define it as

$$
\tau^{(2)}(\widehat{K} ; \Lambda):=\sum_{j}(-1)^{j+1} j \int_{\mathbf{z} \in\left(S^{1}\right)^{m}} \log \left|\operatorname{det} \Delta_{j}(\mathbf{z})\right| d \mathbf{z},
$$

the integral being taken with respect to the invariant probability measure on the compact torus $\left(S^{1}\right)^{m}$.

For example, let $k$ be an oriented knot with exterior $V=\mathbb{S}^{3}-$ int $N(k)$, where $N(k)$ is a regular neighbourhood of $k$. The meridianal generator of the knot group $\pi_{1}\left(\mathbb{S}^{3}-k\right)$ represents a distinguished generator $t$ for its abelianization. We identify this generator with the standard generator 1 of $\mathbb{Z}$. Fix $K$, a triangulation of $V$, as a finite polyhedron and let $\widehat{K}$ be its maximal abelian cover corresponding to the kernel of $\pi_{1}(\mathbb{S}-k) \rightarrow \mathbb{Z}$. Let $\Delta$ be the Alexander polynomial of $k$. It is never identically vanishing and $\widehat{K}$ is $\ell^{2}$-acyclic; see [52] for more details. It is well known (see e.g. [44, (8.2)]) that

$$
\tau^{(2)}(\widehat{K} ; \mathbb{Z})=-\log M(\Delta) .
$$


Theorem 7.3 (Growth of torsion and regulators in abelian covers). With the notation as above, suppose that $\widehat{K}$ is $\ell^{2}$-acyclic. Then

$$
\frac{\log R^{i}\left(K_{N}\right)}{\left[\Lambda: \Lambda_{N}\right]} \rightarrow 0, \quad 0 \leqslant i \leqslant \operatorname{dim}(K) .
$$

Moreover, if $m=1$, we have

$$
\frac{\log T\left(K_{N}\right)}{\left[\Lambda: \Lambda_{N}\right]} \rightarrow-\tau^{(2)},
$$

where the $\tau^{(2)}$ is the $\ell^{2}$-torsion.

Applied to cyclic covers of a knot complement, Theorem 7.3 translates into the theorem of Silver and Williams [65] mentioned in the introduction. It is possible to prove a version of (7.3.2) for $m>1$ replacing lim by lim sup but it is a bit more subtle (see $[43,59])$; also, one can establish $(7.3 .2)$ for general $m$ if we suppose that each det $\Delta_{j}$ is everywhere nonvanishing on $\left(S^{1}\right)^{m}$, this being the analog of 'strongly acyclic'.

Proof. Equation (7.3.1) follows from the subsequent Proposition 7.4, taking into account (7.2.1) and the fact that $\Lambda / \Lambda_{N}$ is abelian.

Now apply (2.2.4) and (2.2.5). They imply, together, that

$$
\log \left(\frac{\prod_{i}^{*} R^{i}\left(K_{N}\right)}{T\left(K_{N}\right)}\right)=\frac{1}{2} \sum_{j}(-1)^{j+1} j\left(\sum_{\zeta^{N}=1} \log \operatorname{det}^{\prime} \Delta_{j}(\zeta)\right)
$$

Equation (7.3.2) then follows from (2.5.3):

Write $\Delta_{j}(z)=a \prod_{j}\left(z-z_{j}\right)$. Note that $z \mapsto \operatorname{det} \Delta_{j}(z)$ is nonnegative on the unit circle and has only finitely many zeros amongst roots of unity, so

$$
\sum_{\zeta^{N}=1} \log \operatorname{det}^{\prime} \Delta_{j}(\zeta)-N \log a-\sum_{z_{j}^{N} \neq 1} \log \left|z_{j}^{N}-1\right|
$$

is bounded as $N \rightarrow \infty$. Thereby, $\frac{1}{N} \sum_{\zeta^{N}=1} \log \operatorname{det}^{\prime} \Delta_{j}(\zeta)$ approaches $\log a+\sum_{j} \log ^{+}\left|z_{j}\right|$, which is the integral of $\log \operatorname{det} \Delta_{j}(z)$ over $S^{1}$.

Proposition 7.4. Let $\delta_{N}$ be the sums of squares of all the dimensions of $\Lambda / \Lambda_{N}$-representations occurring in $H^{i}\left(K_{N}, \mathbb{C}\right)$ (counted without multiplicity). Then,

$$
\left|\frac{\log R^{i}}{\delta_{N}}\right| \leqslant A \log \left[\Lambda: \Lambda_{N}\right]+B,
$$

the constants $A, B$ depending only on $K$.

Note that the proposition does not require $\Lambda / \Lambda_{N}$ to be abelian. The basic idea is to use the action of $\Lambda / \Lambda_{N}$ to split the cochain complex of $K_{N}$ into two pieces: an acyclic piece, and a piece contributing all the cohomology. Under favourable circumstances, the 
latter piece is small, and it follows that $R^{i}$ is small. In practice, we cannot literally split the complex into a direct sum of $\mathbb{Z}$-subcomplexes; nonetheless, we can do so to within a controlled error.

More specifically, the proof will follow from the string of subsequent lemmas: the bound $\frac{\log R^{i}}{\delta_{N}} \geqslant \cdots$ follows by specializing Lemma 7.5 to the case of the cochain complex of $K_{N}$; the inequality $-\frac{\log R^{i}}{\delta_{N}} \geqslant \cdots$ follows from applying that lemma to the dual complex and using Lemma 7.6.

We say that a metrized lattice is integral if the inner product $\langle-,-\rangle$ takes integral values on $L$. Note that a sublattice of an integral lattice is also integral.

Lemma. Any integral lattice has volume $\geqslant 1$.

Lemma. Let $f: A_{1} \rightarrow A_{2}$ be a map of integral lattices of dimensions $\leqslant n$. Suppose that every singular value of $f$ is $\leqslant M$, where we suppose that $M \geqslant 1$. Then,

$$
1 \leqslant \operatorname{vol}(\operatorname{ker} f) \leqslant \operatorname{vol}\left(A_{1}\right) M^{n}
$$

and the same inequality holds for vol(image $f$ ).

Proof. This follows from the prior lemma and (2.1.1).

Lemma. Let $A^{*}$ be a complex, as in (2.2.1). Suppose that every $A^{i}$ is an integral metrized lattice (not necessary of volume 1). Let $v>1, M>1, D$ be such that:

(1) Each $A^{i} \otimes \mathbb{Q}$ has a basis consisting of elements of $A^{i}$ of length $\leqslant v$.

(2) $\operatorname{dim}\left(A^{i}\right) \leqslant D$ for all $i$.

(3) All differentials $d_{i}: A^{i} \rightarrow A^{i+1}$ have all singular values $\leqslant M$.

Then,

$$
R^{i}\left(A^{*}\right) \geqslant(M \nu)^{-D}
$$

Proof. Hadamard's inequality implies that $\operatorname{vol}\left(A^{i}\right) \leqslant v^{D}$ (indeed, if $x_{1}, \ldots, x_{r} \in A^{i}$ form a $\mathbb{Q}$-basis, then $\operatorname{vol}\left(A^{i}\right) \leqslant\left\|x_{1} \wedge \cdots \wedge x_{r}\right\|$, whence the result). The result follows from the prior lemma and (2.2.3).

Lemma 7.5. With notation as in the prior lemma, let $G$ be a finite group of order $|G|$ acting ${ }^{17}$ on $A^{*}$. Let $\Xi$ be the set of all characters of all irreducible $G$-representations that occur in $H^{i}\left(A^{*} \otimes \mathbb{C}\right)$ and let $D$ be the dimension of the $\Xi$-isotypical subspace of $A^{i}$. Then

$$
R^{i}\left(A^{*}\right) \geqslant\left(M v|G|^{5}\right)^{-D} \text {. }
$$

Proof. Set

$$
e_{\Xi}=\sum_{\chi \in \Xi} \chi(1) \sum_{g \in G} \overline{\chi(g)} g \in \mathbb{C}[G] .
$$

17 We understand this to mean that the action on $A^{*} \otimes \mathbb{R}$ is isometric. 
Then, in fact, $e_{\Xi} \in \mathbb{Z}[G]$ : its coefficients are algebraic integers that are fixed under Galois conjugacy. Moreover, $e_{\Xi}^{2}=|G| e_{\Xi}$; indeed, $e_{\Xi} /|G|$ realizes a projection onto the $\Xi$-isotypic space of any $G$-representation.

Since $|\chi(g)| \leqslant|G|$ for all $g,|\Xi| \leqslant|G|$, and each $g \in G$ acts isometrically on $A^{*}$, we see that $e_{\Xi}$ acting on $A^{*}$ increases norms by at most $|G|^{4}$.

Let $\pi: A^{*} \rightarrow A^{*}$ be the endomorphism induced by $e_{\Xi}$; let $B^{*}$ be the image of $\pi$, and $\iota: B^{*} \hookrightarrow A^{*}$ the inclusion. Any 'harmonic form' in $A^{*} \otimes \mathbb{R}$ is fixed by the projection $e_{\Xi} /|G|$ and so belongs to $B^{*} \otimes \mathbb{R}$. Therefore $\iota$ induces an isometric isomorphism

$$
\iota_{* \mathbb{R}}: H^{*}\left(B^{*}, \mathbb{R}\right) \rightarrow H^{*}\left(A^{*}, \mathbb{R}\right) .
$$

We are going to show that $\iota_{* \mathbb{R}} \pi_{* \mathbb{R}}$ - an endomorphism of $H^{*}\left(A^{*}, \mathbb{R}\right)$ - is multiplication by $|G|$. Indeed, let $x \in A^{j}$ be a cycle, so $d x=0$. There exists a cycle $y \in B^{j} \otimes \mathbb{R}$ such that $\iota y=x+d z$, for some $z \in A^{j-1} \otimes \mathbb{R}$. Write $[x],[y]$ for the corresponding cohomology classes with real coefficients, so $\iota_{* \mathbb{R}}[y]=[x]$. Now $\iota_{* \mathbb{R}} \pi_{* \mathbb{R}}[x]$ equals $\iota_{* \mathbb{R}} \pi_{* \mathbb{R}} \iota_{* \mathbb{R}}[y]=|G| \iota_{* \mathbb{R}}[y]=$ $|G| \cdot[x]$. The claim follows.

This implies that the image of $|G| \cdot H^{i}\left(A^{*}, \mathbb{Z}\right)$ inside $H^{i}\left(A^{*}, \mathbb{R}\right)$ is contained in the image of $H^{i}\left(B^{*}, \mathbb{Z}\right)$ inside $H^{i}\left(B^{*}, \mathbb{R}\right) \stackrel{\sim}{\longrightarrow} H^{i}\left(A^{*}, \mathbb{R}\right)$. Thus,

$$
R^{i}\left(A^{*}\right) \geqslant|G|^{-\operatorname{dim} H^{i}} R^{i}\left(B^{*}\right) .
$$

By definition of $B^{i}$ as the image of the endomorphism $\pi$, which increases norms by at most $|G|^{4}, B^{i} \otimes \mathbb{Q}$ is generated as a $\mathbb{Q}$-vector space by elements of $B^{i}$ of length $\leqslant|G|^{4} v$. Finally, $\operatorname{dim} B^{i} \leqslant D$. Combining (7.5.1) and (7.4.1) gives the desired conclusion.

Lemma 7.6. Let $A^{*}$ be a complex, as in (2.2.1), and let $\widehat{A^{*}}$ be the dual complex

$$
0 \leftarrow \widehat{A}^{0} \leftarrow \widehat{A}^{1} \leftarrow \cdots
$$

where $\widehat{A^{j}}=\operatorname{Hom}\left(A^{j}, \mathbb{Z}\right)$, endowed with the dual metric. Then the regulator $\widehat{R}^{j}$ of $\widehat{A^{*}}$ satisfies

$$
\widehat{R}^{j} \cdot R^{j}=1
$$

Proof. In fact, the pairing between $A^{j}$ and $\widehat{A}^{j}$ induces a perfect pairing between the cohomology groups of $A^{*} \otimes \mathbb{C}$ and $\widehat{A^{*}} \otimes \mathbb{C}$, and the image of the cohomology of $A^{*}$ and $\widehat{A^{*}}$ projects to lattices in perfect pairing with respect to this duality.

We have now concluded the proof of Theorem 7.3; let us describe an application.

Corollary 7.7. Let $V$ be a compact 3-manifold which fibres over the circle. Let $S$ be a fibre, $f: S \rightarrow S$ the monodromy map and $P_{f}$ the characteristic polynomial of the linear map induced by $f$ on $H_{1}(S)$. The fibration over the circle induces a map $\pi_{1}(V) \rightarrow \mathbb{Z}$. We denote by $V_{N}$ the corresponding $N$-fold covering of $V$. Then,

$$
\lim _{N \rightarrow+\infty} \frac{\log \left|H_{1}\left(V_{N}\right)_{\text {tors }}\right|}{N}=\log M\left(P_{f}\right) .
$$

Proof. It is a theorem of Lück [49, Theorem 1.40] that the infinite cyclic cover $\widehat{V}$ associated with $\pi_{1}(V) \rightarrow \mathbb{Z}$ is $\ell^{2}$-acyclic. We may thus apply Theorem 7.3 . Here - as 
in the case of knot complements - the $\ell^{2}$-torsion of $\widehat{V}$ is equal to $-\log M(\Delta)$ where $\Delta$ - the natural generalization of the Alexander polynomial - is equal to $P_{f}$. This proves the corollary.

\subsection{Asymptotics of torsion}

We now work with general $\Lambda$. It is possible to define the combinatorial $\ell^{2}$-torsion $\tau^{(2)}(\widehat{K} ; \Lambda)$ of $\widehat{K}$ in a manner generalizing $(7.2 .2)$ in the case $\Lambda=\mathbb{Z}$.

Question. Suppose that $\widehat{K}$ has trivial $\ell^{2}$-homology; under what circumstances does the sequence

$$
\frac{\log T\left(K_{N}\right)}{\left[\Gamma: \Gamma_{N}\right]}
$$

converge to $-\tau^{(2)}(\widehat{K} ; \Lambda)$ as $N \rightarrow+\infty$ ?

This is true if $\widehat{K}$ is 'strongly acyclic', in that the eigenvalues of each $\Delta_{j}$ on each $K_{N}$ are uniformly separated from zero. We do not know of any example where this is so besides those already discussed.

\section{Examples}

\subsection{The existence of strongly acyclic bundles}

Let the notation be as in Theorem 1.4. We shall prove that strongly acyclic bundles always exist when $\delta=1$.

Let $\mathbf{T} \subset \mathbf{G}$ be a maximal torus. Let $E$ be a Galois extension of $\mathbb{Q}$ splitting $\mathbf{T}$. Let $\mathbf{T}_{E}=\mathbf{T} \times_{\mathbb{Q}} E$, and let $X^{*}$ (resp. $X_{+}^{*}$ ) be the character lattice of $\mathbf{T}_{E}$ (resp. the dominant characters). With each $x \in X_{+}^{*}$ there is associated a unique irreducible representation of $\mathbf{G} \times{ }_{\mathbb{Q}} E$, denoted as $\rho_{x}$, with highest weight $x$. There is an associated homomorphism

$$
\mathbf{G} \rightarrow \operatorname{Res}_{E / \mathbb{Q}} \operatorname{GL}\left(W_{x}\right) \hookrightarrow \operatorname{GL}\left(\operatorname{Res}_{E / \mathbb{Q}} W_{x}\right),
$$

where, by $\operatorname{Res}_{E / \mathbb{Q}} W_{x}$, we mean $W_{x}$ considered as a $\mathbb{Q}$-vector space.

Let $\tilde{\rho}_{x}$ be this representation of $\mathbf{G}$ on $\tilde{W}_{x}:=\operatorname{Res}_{E / \mathbb{Q}} W_{x}$. Then $\tilde{W}_{x} \otimes_{\mathbb{Q}} \mathbb{C}$ decomposes into irreducible $\mathbf{G}(\mathbb{C})$-representations; their highest weights are obtained from $x$ by applying the various embeddings $E \hookrightarrow \mathbb{C}$.

We claim that, as long as $x \in X_{+}^{*}$ avoids a finite union of proper hyperplanes, any stable $\mathbb{Z}$-lattice in $\tilde{W}_{x}$ will be strongly acyclic.

In fact, the complexified Cartan involution induces a certain $W$-coset $\mathcal{W} \subset$ $\operatorname{Aut}\left(X^{*}\left(\mathbf{T}_{\mathbb{C}}\right)\right)$ : pick any Cartan-stable maximal torus $\mathbf{T}^{\prime}$ of $\mathbf{G}$ defined over $\mathbb{R}$; the complexified Cartan involution induces an automorphism of $X^{*}\left(\mathbf{T}_{\mathbb{C}}^{\prime}\right)$, and the latter is identified with $X^{*}\left(\mathbf{T}_{\mathbb{C}}\right)$, uniquely up to the action of a Weyl element. According to the lemma below, each $\omega \in \mathcal{W}$ acts nontrivially. Now, by Lemma 4.1, we may take the 'finite union of hyperplanes' to be the preimages of the $\{\operatorname{fix}(\omega): \omega \in \mathcal{W}\}$ under the various maps $X^{*} \rightarrow X^{*}\left(\mathbf{T}_{\mathbb{C}}\right)$ induced by the various embeddings $E \hookrightarrow \mathbb{C}$.

Lemma. Suppose that $\delta=1$. Let $\theta: \mathfrak{g} \rightarrow \mathfrak{g}$ be (the complexification of) a Cartan involution. Let $\mathfrak{t}$ be any $\theta$-stable Cartan subalgebra. Then the action of $\theta$ on $\mathfrak{t}$ does 
not coincide with any Weyl element. In particular, $\theta$ acts nontrivially on the set of isomorphism classes of irreducible $\mathfrak{g}$-modules.

Proof. It suffices to check the case where $\mathfrak{g}_{\mathbb{R}}$ is isomorphic to one of the simple real Lie algebras $\mathfrak{s l}_{3}$ and $\mathfrak{s o}(p, q)$ for $p q$ odd. Now suppose that the assertion is false; we may multiply $\theta$ by an inner automorphism to obtain $\theta^{\prime}$ which acts trivially on $\mathfrak{t}$ and on every positive simple root space. Using the Killing form, we deduce that the action of $\theta^{\prime}$ on every negative simple root space is trivial. Since these, together, generate $\mathfrak{g}$, we conclude that $\theta^{\prime}$ is trivial, and $\theta$ was inner: a contradiction, for in both cases the Cartan involution is not inner.

For example, if $\mathbf{G}$ is given by the units in a nine-dimensional division algebra $D$ over $\mathbb{Q}$, then a congruence lattice in $\mathbf{G}$ is given by $\mathfrak{o}_{D}^{\times}$, the units in a maximal order of $D$; and a strongly acyclic $\mathfrak{o}_{D}^{\times}$-module is given by $\mathfrak{o}_{D}$ itself. Indeed, in this case - taking for $E$ a cubic extension of $\mathbb{Q}$ that splits $D$ - the representation ' $\tilde{W}_{X} \otimes \mathbb{C}$ ', with the notation as previously, is the sum of three standard representations of $\mathbf{G}(\mathbb{C}) \cong \mathrm{SL}_{3}(\mathbb{C})$; moreover, the isomorphism class of the standard representation is not fixed by the Cartan involution; it is interchanged with its dual.

\subsection{Hyperbolic 3-manifolds and the adjoint bundle}

There is a particularly interesting strongly acyclic bundle that exists for certain arithmetic hyperbolic 3-manifolds:

Let $B$ be a quaternion division algebra over an imaginary quadratic field, and $\mathfrak{o}_{B}$ a maximal order. Then $\mathfrak{o}_{B}^{\times}$embeds into $\mathrm{PGL}_{2}(\mathbb{C})$, and acts on $\mathbb{H}^{3}$; let $M$ be the quotient, and $\tilde{M}$ any covering of $M$.

Let $L$ be the set of elements in $\mathfrak{o}_{B}$ of trace 0 , considered as a $\pi_{1}(M)$-module via conjugation. Then $H^{1}(\tilde{M}, L \otimes \mathbb{C})=0$. This is a consequence of Weil local rigidity. In fact, the module $L$ is strongly acyclic, and applying the proof of the theorem reveals that

$$
\frac{\log \left|H_{1}(\tilde{M}, L)\right|}{\operatorname{vol}(\tilde{M})} \longrightarrow \frac{13}{6 \pi}
$$

This has the following consequence: although the defining representation $\pi_{1}(\tilde{M}) \rightarrow$ $\mathrm{SL}_{2}$ does not deform over the complex numbers, it does deform modulo $p$ for many $p$ : indeed, if $p$ divides the order of $H_{1}\left(\pi_{1}(M), L\right)$, this means precisely that there is a nontrivial map

$$
\pi_{1}(M) \rightarrow \mathrm{SL}_{2}\left(\mathbb{F}_{p}[t] / t^{2}\right)
$$

It would be interesting if the existence of 'many' such quotients shed any light on the conjectural failure of property $\tau$; cf. [47].

Remark. For any compact hyperbolic manifold $M$, we may consider the three-dimensional flat complex bundle defined by composing $\rho: \pi_{1}(M) \rightarrow \mathrm{SL}_{2}(\mathbb{C})$, defining the hyperbolic structure, with the adjoint action of $\mathrm{SL}_{2}(\mathbb{C})$. Again, the corresponding local system is acyclic. However, in general, this representation does not 
have an integral structure. It can sometimes be defined over the ring of integers of a number field larger than $\mathbb{Q}$; but the resulting local system of $\mathbb{Z}$-modules is not strongly acyclic.

\subsection{Lifting torsion: hyperbolic $n$-manifolds, $n>3$}

Combined with geometric techniques, one may use Theorem 1.4 to obtain torsion even in certain (nonarithmetic, nonexhaustive) sequences with $\delta=0$.

Let $F$ be a totally real number field and $(V, q)$ be an anisotropic quadratic space over $F$, of signature $(n, 1)$ over one infinite place and definite over all other infinite places. Let $U \subset V$ be a $F$-rational nondegenerate subspace of signature $(3,1)$ over one place at infinity. Let $\sigma$ denote the involutive linear map from $V$ to itself which acts as the identity on $U$ and as minus the identity on $U^{\perp}$.

It is proved in [5] that there exists a - possibly noncongruence - lattice $\Gamma \subset \operatorname{SO}(q, F)$ such that $\Gamma$ retracts onto $\Gamma^{\sigma}$. We denote by

$$
r: \Gamma \rightarrow \Gamma^{\sigma}
$$

the retraction.

The group $\Gamma^{\sigma}$ is a lattice in $\mathrm{SO}(U) \cong \mathrm{SO}_{3,1}$. We let $(L, \rho)$ be a strongly acyclic $\mathbb{Z}\left[\Gamma^{\sigma}\right]$-module. Composing $\rho$ with the retraction $r$ turns $L$ into a $\mathbb{Z}[\Gamma]$-module that we still denote as $\tilde{L}$. It is not arithmetic.

Proposition. (1) There exists a decreasing sequence of finite index subgroups $\Gamma_{N} \leqslant \Gamma$ such that $\left|H_{1}\left(\Gamma_{N}, \tilde{L}\right)_{\text {tors }}\right|$ grows exponentially with $\left[\Gamma: \Gamma_{N}\right]$.

(2) There exists a decreasing sequence of finite index subgroups $\Gamma_{N} \leqslant \Gamma$ such that $\bigcap_{N} \Gamma_{N}=\{1\}$ and

$$
\liminf \frac{\log \left|H_{1}\left(\Gamma_{N}, \tilde{L}\right)_{\text {tors }}\right|}{\operatorname{vol}\left(\Gamma_{N}^{\sigma} \backslash \mathbb{H}^{3}\right)}>0 .
$$

Proof. (1) Theorem 1.4 implies that $\Gamma^{\sigma}$ contains a decreasing sequence of finite index subgroups $\Gamma_{N}^{\sigma} \leqslant \Gamma^{\sigma}$ such that $\left|H_{1}\left(\Gamma_{N}^{\sigma}, L\right)_{\text {tors }}\right|$ grows exponentially with the index $\left[\Gamma^{\sigma}: \Gamma_{N}^{\sigma}\right]$. Let $\Gamma_{N}$ be the preimage of $\Gamma_{N}^{\sigma}$ by the retraction map $r$. Then

$$
H_{1}\left(\Gamma_{N}, \tilde{L}\right) \rightarrow H_{1}\left(\Gamma_{N}^{\sigma}, L\right)
$$

and the conclusion follows.

(2) Let $\Gamma_{N} \leqslant \Gamma$ be any decreasing sequence of finite index subgroups such that $\bigcap_{N} \Gamma_{N}=\{1\}$. It follows from [5] that the map $r$ induces a virtual retraction from $\Gamma_{N}$ onto $\Gamma_{N}^{\sigma}=\Gamma_{N} \cap \Gamma^{\sigma}$. Replacing each $\Gamma_{N}$ by a finite index subgroup, we may thus assume that the retraction map $r$ induces a retraction from $\Gamma_{N}$ onto $\Gamma_{N}^{\sigma}$. This yields injections:

$$
H_{1}\left(\Gamma_{N}^{\sigma}, L\right) \hookrightarrow H_{1}\left(\Gamma_{N}, \tilde{L}\right) .
$$

Now $\bigcap_{N} \Gamma_{N}^{\sigma}=\{1\}$ and $L$ is strongly acyclic. So Theorem 1.4 implies that

$$
\lim \inf \frac{\log \left|H_{1}\left(\Gamma_{N}^{\sigma}, L\right)_{\text {tors }}\right|}{\operatorname{vol}\left(\Gamma_{N}^{\sigma} \backslash \mathbb{H}^{3}\right)}>0 .
$$


Note that in case (1), the intersection $\bigcap_{N} \Gamma_{N}$ is nontrivial. It is equal to the kernel of $r$. Case (2) is reminiscent from the results of Borel, Labesse and Schwermer [9] who construct nontrivial cohomology classes for some series of examples of arithmetic groups as lifts from discrete series in a smaller group.

\subsection{The hyperbolic plane; Shimura varieties}

In the case $G=\mathrm{SL}_{2}(\mathbb{R})$, any torsion-free lattice $\Gamma \leqslant G$ has torsion-free cohomology; indeed, the homology of $\Gamma$ is identified with the homology of the Riemann surface $\Gamma \backslash \mathbb{H}^{2}$.

More generally, it is believed that the cohomology of Shimura varieties (all of which have $\delta=0$; for example, $\mathbf{G}=\operatorname{Sp}_{n}(\mathbb{R})$, or $\mathbf{G}=\mathrm{O}(2 n, m)$, or $\left.\mathbf{G}=U(n, m)\right)$ should have very little torsion - precise statements in a related vein are proposed in [17].

Evidence in this direction is provided by $[12,25]$.

\subsection{Good groups}

Following Serre [64], we say that a group $G$ is $\operatorname{good}^{18}$ if the homomorphism of cohomology groups $H^{n}(\widehat{G}, M) \rightarrow H^{n}(G, M)$ induced by the natural homomorphism $G \rightarrow \widehat{G}$ of $G$ to its profinite completion $\widehat{G}$ is an isomorphism for all $n$ and every finite $G$-module $M$.

Recall two simple facts; see [64, Exercices 1 and 2(b), p. 13].

(1) Goodness is preserved by commensurability.

(2) If $E / N$ is an extension of a good group $G$ by a good group $N$ such that the cohomology groups $H^{q}(N, M)$ are finite for all $q(q \in \mathbb{N})$ and all finite E-modules $M$, then $E$ is good.

If a lattice $\Gamma$ is good then the projective limit

$$
\underset{\overrightarrow{\Gamma^{\prime}}}{\lim _{\overrightarrow{1}}} H^{j}\left(\Gamma^{\prime}, \mathbb{Z} / n \mathbb{Z}\right)
$$

over all its finite index subgroups $\Gamma^{\prime}$ is always zero; in other terms, given any class $\alpha \in H^{j}(\Gamma, M)$ there exists a finite index subgroup $\Gamma_{0} \leqslant \Gamma$ such that $\alpha \mid \Gamma_{0}$ is trivial.

Arithmetic lattices which satisfy the congruence subgroup property (CSP) are not good. On the other hand, Thurston has conjectured that lattices in $\mathrm{SL}_{2}(\mathbb{C})$ are commensurable with the fundamental group of a 3 -manifold fibring over the circle. Such a group is an extension of $\mathbb{Z}$ by either a free group or a surface group. Since these groups are good, it would follow from Thurston's conjecture (and the above two simple facts) that lattices in $\mathrm{SL}_{2}(\mathbb{C})$ are good.

Now, on the one hand it is known [41] that the fundamental group of a hyperbolic 3-manifold whose finite index normal subgroups all have finite abelianization (if such a hyperbolic manifolds exists!) is good. And on the other hand, in [1] Agol introduces a new residual condition (RFRS) under which he proves Thurston's conjecture.

18 This terminology was introduced by Serre in the course of an exercise. Unfortunately it has now become standard. 
Commonly occurring groups which are at least virtually RFRS include surface groups and subgroups of (abstract) right angled reflection groups. Since it follows from $[5,6]$ that arithmetic hyperbolic groups with a nonzero first Betti number are virtual subgroups of (abstract) right angled reflection groups, one gets: ${ }^{19}$

Arithmetic lattices of $\mathrm{SL}_{2}(\mathbb{C})$ are good.

\subsection{Slow growth of coinvariants}

In this section we show (in a special case) - in the notation of Theorem 1.4 - that the growth of the orders of $H_{0}\left(\Gamma_{N}, M\right)_{\text {tors }}$ and $H_{\operatorname{dim}(S)-1}\left(\Gamma_{N}, M\right)_{\text {tors }}$ is at most polynomial in the index. This is compatible with Conjecture 1.3, although it is too easy a case to be serious evidence in that direction; we include it largely for completeness.

We carry this out for a specific family of congruence subgroups; it is very likely that a similar result holds in greater generality. Suppose that $\mathbf{G}, M$ are as in that theorem, $\Gamma$ a congruence subgroup of $\mathbf{G}(\mathbb{Q})$, and $\rho$ the representation of $\mathbf{G}$ on $M \otimes \mathbb{Q}$. We suppose that $\rho$ is faithful, that it has no trivial factors, and that $\Gamma$ is Zariski-dense in $\mathbf{G}$. These conditions guarantee that $H_{0}\left(\Gamma^{\prime}, M\right)$ is torsion for any finite index subgroup $\Gamma^{\prime} \leqslant \Gamma$.

Consider the family of congruence subgroups

$$
\Gamma(N):=\Gamma \cap \rho^{-1}(1+N \operatorname{End}(M)),
$$

where we regard $1+N \operatorname{End}(M)$ as a (principal congruence) subgroup of GL( $M)$. Under these circumstances we show that:

The orders of the (finite) groups $H_{0}(\Gamma(N), M)$ and $H_{\operatorname{dim}(S)-1}(\Gamma(N), M)$ are bounded by a polynomial in N.

We shall prove the result concerning $H_{0}$, the result about $H_{\operatorname{dim}(S)-1}$ following by a dual argument. Note that shrinking $\Gamma$ can only increase the size of $H_{0}$; we begin by replacing $\Gamma$ by a finite index subgroup so that its closure in $\mathbf{G}\left(\mathbb{A}_{f}\right)$ is a product group, i.e., of the form $\prod_{p} K_{p}$, with $K_{p} \subset \mathbf{G}\left(\mathbb{Q}_{p}\right)$ a compact subgroup.

Considering the exact sequence $H_{0}(\Gamma(N), M) \stackrel{\times \ell^{r}}{\longrightarrow} H_{0}(\Gamma(N), M) \rightarrow H_{0}\left(\Gamma(N), M / \ell^{r} M\right)$ we see that

$$
\left|H_{0}(\Gamma(N), M)\right|=\prod_{\ell}\left|H_{0}\left(K_{\ell}[N], M \otimes \mathbb{Z}_{\ell}\right)\right| .
$$

Here, $K_{\ell}[N]=\rho\left(K_{\ell}\right) \cap 1+N \operatorname{End}\left(M \otimes \mathbb{Z}_{\ell}\right)$. In particular, $K_{\ell}[N]=K_{\ell}[1]$ if $\ell$ does not divide $N$. Consequently,

$$
\left|H_{0}(\Gamma(N), M)\right| \leqslant\left|H_{0}(\Gamma(1), M)\right| \cdot \prod_{\ell \mid N}\left|H_{0}\left(K_{\ell}[N], M \otimes \mathbb{Z}_{\ell}\right)\right| .
$$

Fix now a prime $\ell$ dividing $N$. Let $s$ be the $\ell$-valuation of $N$ if $\ell$ is odd; if $\ell=2$, let $s=\max (3,2$-valuation of $N)$. Note that $K_{\ell}[N] \supseteq K_{\ell}\left[\ell^{s}\right]$.

Let $\mathfrak{g}$ be the Lie algebra of $\rho(\mathbf{G})$; we identify it with a subspace of $\operatorname{End}\left(M_{\mathbb{Q}}\right)$, and, because $\rho$ has no invariant subspaces, $\mathfrak{g} M_{\mathbb{Q}}=M_{\mathbb{Q}}$. (Here we write $\mathfrak{g} M_{\mathbb{Q}}$ for the image

${ }^{19} \mathrm{~A}$ particular case is proved in [34]. 
of the action map $\mathfrak{g} \times M_{\mathbb{Q}} \rightarrow M_{\mathbb{Q}}$; we use similar notation in what follows.) Write $\mathfrak{g}_{\mathbb{Z}}=\mathfrak{g} \cap \operatorname{End}(M)$. Then the index of $\left[M: \mathfrak{g}_{\mathbb{Z}} M\right]$ is finite.

Set $\mathfrak{g}\left[\ell^{s}\right]=\mathfrak{g} \cap \ell^{s} \operatorname{End}(M)$ and $M_{\ell}=M \otimes \mathbb{Z}_{\ell}$. Let $U_{\ell}$ be the space spanned by $\left\{g v-v: g \in K_{\ell}\left[\ell^{s}\right], v \in M_{\ell}\right\}$. We claim that

$$
U_{\ell} \supset\left\{X v: X \in \mathfrak{g}\left[\ell^{s}\right], v \in M_{\ell}\right\} .
$$

Indeed, given $X \in \mathfrak{g}\left[\ell^{s}\right]$, set $g=\exp (X)=\sum_{i \geqslant 0} \frac{X^{i}}{i !} \in \mathrm{GL}\left(M_{\ell}\right)$; the series converges in $\operatorname{End}\left(M_{\ell}\right)$. One checks that in fact $g \in K_{\ell}\left[\ell^{s}\right]$. Then, for $v \in M_{\ell}$,

$$
X v=\sum_{i \geqslant 1}(-1)^{i} \frac{(g-1)^{i} v}{i}=(g-1)\left(\sum_{i \geqslant 1}(-1)^{i} \frac{(g-1)^{i-1} v}{i}\right) .
$$

Since $\frac{(g-1)^{i-1} v}{i} \in M_{\ell}$ for each $i \geqslant 1$, we see indeed that $X v \in U_{\ell}$. Since $H_{0}\left(K_{\ell}[N], M\right)$ is isomorphic to a quotient of $M / U_{\ell}$, we deduce that

$$
\left|H_{0}(\Gamma(N), M)\right| \leqslant\left|H_{0}(\Gamma(1), M)\right| \cdot(8 N)^{\operatorname{rank}(M)^{2}} \cdot\left[M: \mathfrak{g}_{\mathbb{Z}} M\right] .
$$

\section{Questions}

\subsection{Trivial coefficients}

For simplicity, we confine ourselves to hyperbolic 3-manifolds and phrase our results in terms of homology, since in this case the interesting torsion is in $H_{1}$, which is simply the abelianized fundamental group.

If one considers trivial coefficients, the Cheeger-Müller theorem expresses the product

$$
\left|H_{1}(M, \mathbb{Z})_{\text {tors }}\right| \cdot R
$$

as a regularized Laplacian determinant; here $R$ is defined as follows: take $\omega_{1}, \ldots, \omega_{N}$ an $L^{2}$-basis of harmonic 1 -forms, $\gamma_{1}, \ldots, \gamma_{N}$ a basis for $H_{1}(M, \mathbb{Z})$, and put $R:=$ $\operatorname{det}\left(\int_{\gamma_{i}} \omega_{j}\right)_{1 \leqslant i, j \leqslant N}^{-2}$.

Thus, to understand whether or not the torsion grows, we need to understand whether or not the quantity $R$ can be very large - in particular, can it be exponentially large in the volume of $M$ ? (Note, however, that even a complete understanding of $R$ would not settle the question of torsion growth; there remains the closely related and extremely difficult issue of small eigenvalues of the Laplacian).

In general, we see no reason that $R$ should be small. However, in the arithmetic case, numerical experiments suggest that $H_{1}(M, \mathbb{Z})_{\text {tors }}$ is large whether or not the $H_{1}(M, \mathbb{Q})$ is zero. Moreover, we saw in the combinatorial case (Lemma 7.5) that one may prove upper bounds for (combinatorial) regulators using the auxiliary structure of a group action; one may suspect that Hecke operators might play a similar role. Accordingly, we formulate: 
Conjecture 9.2. Suppose that $M_{k}$ is a sequence of congruence arithmetic hyperbolic 3-manifolds, of injectivity radius approaching $\infty$. Then

$$
\lim _{k} \frac{\log R\left(M_{k}\right)}{\operatorname{vol}\left(M_{k}\right)}=0
$$

To clarify the difficulty here, we point out that we do not know how to prove this even if, for example, we know that $\operatorname{dim} H_{1}\left(M_{k}, \mathbb{C}\right)=1$ for every $k$. Rather, the difficulty is this: if $\operatorname{dim} H_{1}(M, \mathbb{Q})=1$ it is usually rather easy to find an explicit element $\gamma \in \pi_{1}(M)$ whose image generates $H_{1}(\mathbb{Q})$ but it may not generate $H_{1}(M, \mathbb{Z})$. (It is likely that this is related to the Gromov norm on $\mathrm{H}_{2}$.)

As remarked, we certainly do not conjecture this in the nonarithmetic case, and in fact are more inclined to think it false. In a related vein, we suspect that the answer to the following question is YES, in contrast to the arithmetic case.

Question. Do there exist hyperbolic 3-manifolds of arbitrarily large injectivity radius with torsion-free $H_{1}$ ?

\subsection{Weight 1 modular forms, modulo $p$}

Now suppose that $G=\mathrm{SL}_{2}(\mathbb{R})$. Curiously enough, even though $\operatorname{rank}(G)-\operatorname{rank}(K)=0$ and there is no torsion in the homology of (torsion-free) lattices, there is a natural candidate for a phenomenon that 'mirrors' the exponential growth of torsion. This was observed in the joint work of the second author with Calegari; cf. [20]. We explicate it only for the most concrete case:

Let $\pi: X_{0}(N) \rightarrow \operatorname{Spec} \mathbb{Z}\left[\frac{1}{6 N}\right]$ be the modular curve of level $N$ - regarded as a $\mathbb{Z}\left[\frac{1}{6 N}\right]$-scheme - and let $\Omega=\Omega_{\mathscr{E} / X_{0}(N)}^{1}$ be the bundle of relative 1 -forms corresponding to the universal generalized elliptic curve; let $\omega$ be its pullback to $X_{0}(N)$ by means of the zero-section. Set $M_{1}(N)=\mathrm{R}^{1} \pi_{*} \omega$, which we identify (via its global sections) with a finite rank module over $\mathbb{Z}[1 / N]$.

Question. Does the size $\left|M_{1}(N)_{\text {tors }}\right|$ grow exponentially with $N$ ?

Suppose that $M_{1}(N)[p]$ is nonzero; the long exact sequence in cohomology associated with the sequence of sheaves $\omega \stackrel{\times p}{\longrightarrow} \omega \rightarrow \omega / p \omega$ shows that there is a level $N$, weight 1 modular form modulo $p$ that fails to lift to characteristic 0. Parallel to the analytic torsion case, the size of $M_{1}(N)$ is related to the determinant of a holomorphic Laplacian.

\subsection{The $p$-part}

Throughout this paper, we have been concerned with the 'crude' question of the size of torsion homology; we may also ask, more finely, about its group structure.

With the notation as in Conjecture 1.3, let $p$ be a prime. If $X$ is a finite abelian group, we denote by $X_{p}$ the set of $x \in X$ such that $p^{n} x=0$ for some $n \geqslant 0$.

Question. What can one say about limit $\lim \frac{\log \left|H_{j}\left(\Gamma_{N}, M\right)_{p}\right|}{\left[\Gamma: \Gamma_{N}\right]}$ ?

See Silver and Williams [66, Theorem 4.2] for cyclic covers. The question may be of most interest when the $\Gamma_{N}$ are obtained by adding $p$-power level structure to $\Gamma_{1}$. In this case, one expects the asymptotic behaviour of $H_{j}(\ldots)_{p}$ to be related to the dimension of a suitable eigenvariety. We refer the reader to the works of Calegari and 
Emerton $[16,18]$ for further discussion of this. These suggest that the torsion predicted by our Conjecture 1.3 involves larger and larger sporadic primes.

In a different vein, we may ask:

Question. What is the distribution of the isomorphism class of $H_{i}(\Gamma, M)_{p}$ when one varies $\Gamma$ through arithmetic subgroups?

This question is very vague, of course; one needs to be specific about the variation of $\Gamma$. In particular, it is desirable to ensure that the normalizer of each $\Gamma$ is as small as possible, so that the picture is not clouded by extra automorphisms. For example, the normalizers of the standard subgroups $\Gamma_{0}(\mathfrak{p})$ of $\mathrm{SL}_{2}(\mathbb{Z}[i])$, where $\mathfrak{p}$ is a prime of $\mathbb{Z}[i]$, might be a suitable family.

For example, let us suppose that we have fixed a family $\left\{\Gamma_{\alpha}\right\}_{\alpha \in A}$ of arithmetic subgroups, and consider

$$
X_{k}:=\text { fraction of } \alpha \in A \text { for which } \operatorname{dim} H_{i}(M) \otimes \mathbb{F}_{p}=k .
$$

If the distribution is governed by 'Cohen-Lenstra heuristics' (see below), we expect that $X_{k}$ might decay very rapidly as $k$ grows - as $p^{-k^{2}}$. On the other hand, the existence of Hecke operators may cause a substantial deviation (this phenomenon may be similar to the observed difference in eigenvalue spacings between arithmetic and nonarithmetic Fuchsian groups [61]); one might perhaps expect that $X_{k}$ would decay rather as $p^{-k}$. We do not know; for a certain model of (usually nonarithmetic) hyperbolic 3-manifolds this question has been studied by Dunfield and Thurston [26]. The probability that the homology group of a random 3 -manifold $H_{1}\left(M, \mathbb{F}_{p}\right)$ is nonzero is of size roughly $p$. Again, because the series of $1 / p$ over primes diverges, this suggests that the first homology of a random 3-manifold is typically finite, but is divisible by many primes; see [42] for a quantified version of this.

Remark. Let $t \geqslant 0$. There is a unique probability distribution $\mu_{t}$ on isomorphism classes of finite abelian p-groups - the Cohen-Lenstra distribution with parameter $t$ (cf. [24]) - characterized in the following equivalent ways:

(1) The distribution of the cokernel of a random map $\mathbb{Z}_{p}^{N+t} \rightarrow \mathbb{Z}_{p}^{N}$ (random according to the additive Haar measure on the space of such maps) approaches $\mu_{t}$ as $N \rightarrow \infty$.

(2) $\mu_{t}(A)$ is proportional to $|A|^{-t}|\operatorname{Aut}(A)|^{-1}$.

(3) Let $G$ be any finite abelian group; the expected number of homomorphisms from a $\mu_{t}$-random group into $G$ equals $|G|^{-t}$.

Acknowledgements. The first author (N.B.) would like to thank Pierre Lochak who taught him goodness and Julien Marché who told him about Silver and Williams' theorem.

The second author (A.V.) would like to express his deep gratitude to Frank Calegari for many conversations related to the subject material. Besides this, Calegari's ideas have played an important role in stimulating the present work: the idea that torsion should grow fast was suggested by the unpublished data of Calegari and Dunfield as well as work of Calegari and Emerton. In addition, the use of analytic torsion 
in such arithmetic questions was already used in the joint work with Calegari [20]. A.V. also expresses his gratitude to Université Pierre et Marie Curie for their kind hospitality in the summer of 2008, to Laurent Clozel who suggested that it would be interesting to study torsion in the homology of arithmetic groups, and to Avner Ash for encouragement and helpful remarks.

Finally, we would like to thank Jordan Ellenberg, Matt Emerton and Werner Müller for their comments on, and corrections to, a draft form of this paper.

We gratefully acknowledge the funding agencies that have supported our work. A.V. was partially supported by the Sloan foundation, the Packard Foundation and by an NSF grant. N.B. is a member of the Institut Universitaire de France.

\section{References}

1. IAn Agol, Criteria for virtual fibering, J. Topol. 1(2) (2008), 269-284.

2. Avner Ash AND Warren Sinnott, An analogue of Serre's conjecture for Galois representations and Hecke eigenclasses in the $\bmod p$ cohomology of $\operatorname{GL}(n, \mathbb{Z})$, Duke Math. J. 105 (2000).

3. Darrin Doud, Avner Ash AND DAvid Pollack, Galois representations with conjectural connections to arithmetic cohomology, Duke Math. J. 112 (2002), 521-579.

4. Dan Barbasch and Henri Moscovici, $L^{2}$-index and the Selberg trace formula, J. Funct. Anal. 53(2) (1983), 151-201.

5. Nicolas Bergeron, Frédéric Haglund and Daniel T. Wise, Hyperplane sections in arithmetic hyperbolic manifolds, J. Lond. Math. Soc. (2) 83(2) (2011), 431-448.

6. Nicolas Bergeron and Daniel T. Wise, 2012 A boundary criterion for cubulation. Amer. J. Math. 134(3), in press.

7. Manjul Bhargava, Mass formulae for extensions of local fields, and conjectures on the density of number field discriminants, Int. Math. Res. Not. IMRN (17) (2007), 20 Art. ID rnm052.

8. Jean-Michel Bismut and Weiping Zhang, An extension of a theorem by Cheeger and Müller, Astérisque (205) (1992), 235. With an appendix by François Laudenbach.

9. A. Borel, J.-P. LABesse AND J. Schwermer, On the cuspidal cohomology of $S$-arithmetic subgroups of reductive groups over number fields, Compos. Math. 102(1) (1996), 1-40.

10. A. Borel AND N. Wallach, Continuous cohomology, discrete subgroups, and representations of reductive groups, second edition, Mathematical Surveys and Monographs, Volume 67 (American Mathematical Society, Providence, RI, 2000).

11. Nicolas Bourbaki, Lie groups and Lie algebras. Chapters 1-3, in Elements of mathematics (Berlin). (Springer-Verlag, Berlin, 1998); Translated from the French, Reprint of the 1989 English translation.

12. Pascal Boyer, Pour $l \neq 2$, la $\mathbb{Z}_{l}$-cohomologie du modèle de Deligne-Carayol et de quelques variétés simples de Shimura de Kottwitz est sans torsion, preprint.

13. Kevin Buzzard, Fred Diamond and Frazer Jarvis, On Serre's conjecture for mod $\ell$ Galois representations over totally real fields, Duke Math. J. 155(1) (2010), 105-161.

14. Kevin Buzzard And Toby Gee, 2011 The conjectural connections between automorphic representations and galois representations. In Proceedings of the LMS Durham Symposium, in press.

15. Frank Calegari and Nathan M. Dunfield, Automorphic forms and rational homology 3-spheres, Geom. Topol. 10 (2006), 295-329 (electronic). 
16. Frank Calegari and Matthew Emerton, Completed cohomology, a survey. Non-abelian Fundamental Groups and Iwasawa Theory, CUP, in press.

17. Frank Calegari and Matthew Emerton, Bounds for multiplicities of unitary representations of cohomological type in spaces of cusp forms, Ann. of Math. (2) 170(3) (2009), 1437-1446.

18. Frank Calegari and Matthew Emerton, Mod- $p$ cohomology growth in $p$-adic analytic towers of 3-manifolds, Groups Geom. Dyn. 5(2) (2011), 355-366.

19. Frank Calegari and Barry Mazur, Nearly ordinary Galois deformations over arbitrary number fields, J. Inst. Math. Jussieu 8(1) (2009), 99-177.

20. Frank Calegari and Akshay Venkatesh, Toward a torsion Jacquet-Langlands correspondence, in preparation.

21. Jeff Cheeger, Analytic torsion and the heat equation, Ann. of Math. (2) 109(2) (1979), 259-322.

22. Siu Yuen Cheng, Peter Li and Shing Tung Yau, On the upper estimate of the heat kernel of a complete Riemannian manifold, Amer. J. Math. 103(5) (1981), 1021-1063.

23. Bryan Clair And Kevin Whyte, Growth of Betti numbers, Topology 42(5) (2003), $1125-1142$.

24. H. Cohen And H. W. Lenstra JR., Heuristics on class groups of number fields, in Number theory. (Noordwijkerhout, 1983).

25. Mladen Dimitrov, On Ihara's lemma for Hilbert modular varieties, Compos. Math. 145(5) (2009), 1114-1146.

26. Nathan M. Dunfield and William P. Thurston, Finite covers of random 3-manifolds, Invent. Math. 166(3) (2006), 457-521.

27. J. Elstrodt, F. Grunewald and J. Mennicke, PSL(2) over imaginary quadratic integers, in Arithmetic Conference (Metz, 1981), Astérisque, Volume 94. pp. 43-60 (Soc. Math., France, Paris, 1982).

28. Matthew Emerton, On the interpolation of systems of eigenvalues attached to automorphic Hecke eigenforms, Invent. Math. 164(1) (2006), 1-84.

29. Graham Everest and Thomas WARD, Heights of polynomials and entropy in algebraic dynamics, in Universitext. (Springer-Verlag London Ltd., London, 1999).

30. L. M. Figueiredo, Serre's conjecture for imaginary quadratic fields, Compos. Math. 118(1) (1999), 103-122.

31. A. O. GeL'Fond, Transcendental and algebraic numbers (Dover Publications Inc., New York, 1960), Translated from the first Russian edition by Leo F. Boron.

32. Benedict Gross, Odd galois representations, preprint (http://www.math.harvard.edu/ $\sim$ gross/preprints/Galois_Rep.pdf).

33. Benedict H. Gross, On the Satake isomorphism, in Galois representations in arithmetic algebraic geometry (Durham, 1996), London Math. Soc. Lecture Note Ser., Volume 254. pp. 223-237 (Cambridge Univ. Press, Cambridge, 1998).

34. F. Grunewald, A. Jaikin-Zapirain and P. A. Zalesskit, Cohomological goodness and the profinite completion of Bianchi groups, Duke Math. J. 144(1) (2008), 53-72.

35. Harish-Chandra, Harmonic analysis on real reductive groups. I. The theory of the constant term, J. Funct. Anal. 19 (1975), 104-204.

36. HARISh-Chandra, Harmonic analysis on real reductive groups. III. The Maass-Selberg relations and the Plancherel formula, Ann. of Math. (2) 104(1) (1976), 117-201.

37. Henryk Hecht And Wilfried Schmid, A proof of Blattner's conjecture, Invent. Math. 31(2) (1975), 129-154.

38. Florian Herzig, The weight in a Serre-type conjecture for tame $n$-dimensional Galois representations, Duke Math. J. 149(1) (2009), 37-116. 
39. Anthony W. KnApP, Representation theory of semisimple groups, in Princeton landmarks in mathematics (Princeton University Press, Princeton, NJ, 2001), An overview based on examples, Reprint of the 1986 original.

40. G. KNIEPER, On the asymptotic geometry of nonpositively curved manifolds, Geom. Funct. Anal. 7(4) (1997), 755-782.

41. Dessislava H. Kochloukova and Pavel A. Zalesskit, Profinite and pro- $p$ completions of Poincaré duality groups of dimension 3, Trans. Amer. Math. Soc. 360(4) (2008), 1927-1949.

42. Emmanuel Kowalski, The large sieve, property $(\mathrm{T})$ and the homology of dunfield-thurston random 3-manifolds (unpublished note available at http://www. ufr-mi.u-bordeaux.fr/ kowalski/notes-unpublished.html).

43. Thang Le, Homology torsion growth and mahler measure (arXiv:1010.4199).

44. Weiping Li and Weiping Zhang, An $L^{2}$-Alexander invariant for knots, Commun. Contemp. Math. 8(2) (2006), 167-187.

45. D. A. Lind, Dynamical properties of quasihyperbolic toral automorphisms, Ergodic Theory Dynam. Syst. 2(1) (1982), 49-68.

46. John Lотт, Heat kernels on covering spaces and topological invariants, J. Differential Geom. 35(2) (1992), 471-510.

47. A. LubotzKy AND B. Weiss, Groups and expanders, in Expanding graphs (Princeton, NJ, 1992), DIMACS Ser. Discrete Math. Theoret. Comput. Sci., Volume 10, pp. 95-109. (Amer. Math. Soc., Providence, RI, 1993).

48. W. LüCK, Approximating $L^{2}$-invariants by their finite-dimensional analogues, Geom. Funct. Anal. 4(4) (1994), 455-481.

49. WOLFGAng LÜCK, $L^{2}$-invariants: theory and applications to geometry and $K$-theory, Ergebnisse der Mathematik und ihrer Grenzgebiete. 3. Folge. A Series of Modern Surveys in Mathematics [Results in Mathematics and Related Areas. 3rd Series. A Series of Modern Surveys in Mathematics], Volume 44 (Springer-Verlag, Berlin, 2002).

50. I. G. Macdonald, The volume of a compact Lie group, Invent. Math. 56(2) (1980), 93-95.

51. Simon Marshall and Werner Müller, On the torsion in the cohomology of arithmetic hyperbolic 3-manifolds (arXiv:1103.2262).

52. John Milnor, A duality theorem for Reidemeister torsion, Ann. of Math. (2) 76 (1962), 137-147.

53. WERnER MülleR, The asymptotics of the ray-singer analytic torsion of hyperbolic 3-manifolds (arXiv:1003:5168).

54. Werner Müller, Analytic torsion and $R$-torsion of Riemannian manifolds, Adv. Math. 28(3) (1978), 233-305.

55. WeRner MÜLLeR, Analytic torsion and $R$-torsion for unimodular representations, J. Amer. Math. Soc. 6(3) (1993), 721-753.

56. Jürgen Neukirch, Alexander Schmidt And Kay Wingberg, Cohomology of number fields, second edition, Grundlehren der Mathematischen Wissenschaften [Fundamental Principles of Mathematical Sciences], Volume 323 (Springer-Verlag, Berlin, 2008).

57. Martin Olbrich, $L^{2}$-invariants of locally symmetric spaces, Doc. Math. 7 (2002), 219-237 (electronic).

58. R. Parthasarathy, Criteria for the unitarizability of some highest weight modules, Proc. Indian Acad. Sci. Sect. A Math. Sci. 89(1) (1980), 1-24.

59. Jean Raimbault, Exponential growth of torsion in abelian coverings. Algebraic and Geometric Topology, in press (arXiv:1012.3666). 
60. D. B. RAY And I. M. Singer, R-torsion and the Laplacian on Riemannian manifolds, Adv. Math. 7 (1971), 145-210.

61. Peter Sarnak, Arithmetic quantum chaos. 1992 Schur lectures.

62. R. T. SEELEY, Complex powers of an elliptic operator, in Singular integrals (Proc. Sympos. Pure Math., Chicago, Ill., 1966), pp. 288-307. (Amer. Math. Soc., Providence, RI, 1967).

63. Mehmet Haluk Şengün, On the integral cohomology of Bianchi groups, Exp. Math. 20(4) (2011), 487-505.

64. Jean-Pierre Serre, Cohomologie galoisienne, fifth edition, Lecture Notes in Mathematics, Volume 5 (Springer-Verlag, Berlin, 1994).

65. Daniel S. Silver and Susan G. Williams, Mahler measure, links and homology growth, Topology 41(5) (2002), 979-991.

66. Daniel S. Silver and Susan G. Williams, Torsion numbers of augmented groups with applications to knots and links, Enseign. Math. (2) 48(3-4) (2002), 317-343.

67. Richard TAYlor, On Congruences between modular forms. PhD thesis (available on http://www.math.harvard.edu/ rtaylor/).

68. David A. Vogan JR. And GregG J. Zuckerman, Unitary representations with nonzero cohomology, Compos. Math. 53(1) (1984), 51-90.

69. Nolan R. Wallach, Real reductive groups. I, Pure and Applied Mathematics, Volume 132 (Academic Press Inc., Boston, MA, 1988). 